\title{
Nonlinear parabolic SPDEs involving Dirichlet operators
}

\author{
Tomasz Klimsiak and Andrzej Rozkosz
}

\begin{abstract}
We study the problem of existence, uniqueness and regularity of probabilistic solutions of the Cauchy problem for nonlinear stochastic partial differential equations involving operators corresponding to regular (nonsymmetric) Dirichlet forms. In proofs we combine the methods of backward doubly stochastic differential equations with those of probabilistic potential theory and Dirichlet forms.
\end{abstract}

\section{Introduction}

In the present paper we are concerned with the problem of existence, uniqueness and regularity of probabilistic solutions of stochastic partial differential equations (SPDEs for short) of the form

$$
d u(t)=\left(A_{t} u+f(t, x, u)\right) d t+\tilde{g}(t, x, u) d B_{t}, \quad u(0)=\varphi .
$$

In (1.1), $B$ is some $Q$-Wiener process and $A_{t}, t \in[0, T]$, are operators associated with some family of regular (nonsymmetric) Dirichlet forms satisfying mild regularity assumptions. These assumptions are automatically satisfied if $A_{t}=A, t \in[0, T]$. Therefore our results apply in particular to equations (1.1) with $A_{t}$ replaced by any operator $A$ corresponding to a regular Dirichlet form. The class of such operators is quite wide. It contains both local operators, whose model example is the Laplacian $\Delta$, and nonlocal operators, whose model example is the fractional Laplacian $\Delta^{\alpha / 2}$ with $\alpha \in(0,2)$. Other interesting examples are to be found for instance in $[8,13,14,16]$. An important example of the family of operators depending on $t$ and satisfying our regularity assumptions is the family of uniformly elliptic operators of the form

$$
A_{t} u=\sum_{i, j=1}^{d}\left(a_{i j}(t, x) u_{x_{i}}\right)_{x_{j}}
$$

with ellipticity constant not depending on $t$. Actually, in case $A_{t}$ are of the form (1.2), we consider equations more general then (1.1) with coefficients $f, \tilde{g}$ depending on $u$ and its gradient $\nabla u$.

2010 Mathematics Subject Classification: Primary 60H15; Secondary 60H10, 60H30, 35R60.

Key words and phrases: Stochastic partial differential equation, Backward doubly stochastic differential equation, Dirichlet form. 
As for $\varphi, f, \tilde{g}$, we assume that $\varphi, f(\cdot, \cdot, 0), \tilde{g}(\cdot, \cdot, 0)$ are square-integrable, $f(t, x, \cdot)$ is continuous and monotone (no assumption on the growth of $f(t, x, \cdot)$ is imposed) and $\tilde{g}(t, x, \cdot)$ is Lipschitz -continuous. In the case where $f, \tilde{g}$ depend on $u$ and $\nabla u$, we also assume that they are Lipschitz-continuous with respect to $\nabla u$.

To study (1.1) we develop the approach used successfully in $[12,13,14]$ to investigate Sobolev space solutions of semilinear PDEs with operators corresponding to Dirichlet forms. In those papers PDEs are studied by the methods of the theory of backward stochastic differential equations (BSDEs for short) combined with those of the probabilistic potential theory and Dirichlet forms. In the present paper the strategy for studying SPDEs is similar. The major difference is that now we use backward doubly stochastic differential equations (BDSDEs for short) instead of BSDEs. The idea of studying nonlinear SPDEs via BDSDEs goes back to [20]. In [20] classical solutions of equations with nondivergence form operators with regular coefficients are considered. Our approach to (1.1) was also motivated by the desire to develop the ideas of [20] to encompass a broader class of operators and to study Sobolev space solutions.

As a matter of fact, we study the following Cauchy problem with terminal condition:

$$
d u(t)=-\left(A_{t} u+f(t, x, u)\right) d t-g(t, x, u) d^{\dagger} \beta_{t}, \quad u(T)=\varphi .
$$

Here $g=\left(g_{k}\right)$ is a sequence of real functions on $\Omega \times(0, T] \times E \times \mathbb{R}$ determined by $\tilde{g}$ and $Q, \beta=\left(\beta_{k}\right)$ is a sequence of one-dimensional mutually independent standard Wiener processes defined on some probability space $(\Omega, \mathcal{F}, P)$ and $g d^{\dagger} \beta_{t}=\sum_{k=1}^{\infty} g_{k} d^{\dagger} \beta_{t}^{k}$, where $g_{k} d^{\dagger} \beta_{t}^{k}$ denotes the backward Itô integral. The results for (1.3) can be easily translated into results for (1.1). However, since we heavily rely on the theory of BDSDEs, problem (1.3) is much more convenient to deal with.

Roughly speaking, our strategy for investigating (1.3) consists of two steps. Suppose that the operators $A_{t}$ are associated with some family of Dirichlet forms $\left\{B^{(t)}\right\}$ on $L^{2}(E ; m)$ with common domain $V$ and let $\mathcal{E}$ be the time-dependent Dirichlet form determined by $\left\{B^{(t)}\right\}$. Denote by $\mathbf{M}=\left\{\left(\mathbf{X}, P_{z}\right) ; z \in \mathbb{R} \times E\right\}$ a time-space Markov process with life time $\zeta$ associated with $\mathcal{E}$. In the first step we prove that there exists an exceptional set $N \subset E_{0, T}:=(0, T] \times E$ and a pair of processes $(Y, M)$ such that for every $z \in E_{0, T} \backslash N$ the process $M$ is a martingale under $P \otimes P_{z}$ and $(Y, M)$ is a unique solution of the BDSDE

$$
\begin{aligned}
Y_{t}= & \varphi\left(\mathbf{X}_{T_{\iota}}\right)+\int_{t}^{\zeta \wedge T_{\iota}} f\left(\mathbf{X}_{r}, Y_{r}\right) d r+\int_{t}^{\zeta \wedge T_{\iota}} g\left(\mathbf{X}_{r}, Y_{r}\right) d^{\dagger} \beta_{r}^{\iota} \\
& -\int_{t}^{\zeta \wedge T_{\iota}} d M_{r}, \quad t \in\left[0, \zeta \wedge T_{\iota}\right], \quad P \otimes P_{z} \text {-a.s. },
\end{aligned}
$$

where $T_{\iota}=T-\iota(0)$. Here $\iota$ is the uniform motion to the right (in particular, $\iota(0)=s$ under $P_{z}$ with $\left.z=(s, x)\right)$ and $\beta_{t}^{\iota}=\beta_{t+\iota(0)}, t \geq 0$. In fact, we show that

$$
Y_{t}=u\left(\mathbf{X}_{t}\right), \quad t \in\left[0, \zeta \wedge T_{\iota}\right]
$$

for some $u: \Omega \times E_{0, T} \rightarrow \mathbb{R}$ such that $u(\omega ; \cdot)$ is quasi-continuous (with respect to the capacity associated with $\mathcal{E}$ ). Therefore putting $t=0$ in (1.4) and taking the expectation with respect to $P_{z}$ we see that $u$ satisfies the nonlinear Feynman-Kac formula

$$
u(z)=E_{z}\left(\varphi\left(\mathbf{X}_{T_{\iota}}\right)+\int_{0}^{\zeta \wedge T_{\iota}} f\left(\mathbf{X}_{t}, u\left(\mathbf{X}_{t}\right)\right) d t+\int_{0}^{\zeta \wedge T_{\iota}} g\left(\mathbf{X}_{t}, u\left(\mathbf{X}_{t}\right)\right) d^{\dagger} \beta_{t}^{\iota}\right) .
$$


In fact, a quasi-continuous $u$ satisfying (1.5) is unique and we call it the (probabilistic) solution of (1.1). The second step is to use (1.5) to derive regularity properties of $u$. Our main result says that there is $c>0$ such that

$$
\begin{aligned}
& \sup _{0 \leq t \leq T} E\|u(t)\|_{L^{2}(E ; m)}^{2}+E \int_{0}^{T}\|u(t)\|_{V}^{2} d t \\
& \quad \leq c E\left(\|\varphi\|_{L^{2}(E ; m)}^{2}+\int_{0}^{T}\left(\|f(t, \cdot, 0)\|_{L^{2}(E ; m)}^{2}+\sum_{k=1}^{\infty}\left\|g_{k}(t, \cdot, 0)\right\|_{L^{2}(E ; m)}^{2}\right) d t\right) .
\end{aligned}
$$

The problems of existence and uniqueness of solutions of (1.1) have been the subject of numerous papers. Here let us mention important papers [21, 22] dealing with mild solutions of equations of the form (1.1) with $A_{t}=\Delta$ and $B$ being a spatially homogeneous Wiener process. We also refer to $[21,22]$ for many bibliographic comments on (1.1) with $A_{t}=\Delta$. Note that in $[21,22]$ the "semigroup approach" to (1.1) is used. For results on (1.1) (with $A_{t}$ being local operators) which can be obtained by using the "variational approach", see $[15,25]$ and the references therein. Solutions in the sense defined by Walsh [29] are considered for instance in [1,9] (see also the expository paper $[5])$.

The approach of [20], via BDSDE, was developed in several papers. In $[2,3,30,31]$ it is assumed that the operators $A_{t}$ are the same as in [20], i.e. second order operators in nondivergence form with coefficients having some regularity properties. In $[2,31]$ under the assumption that $f, \tilde{g}$ are Lipschitz continuous a stochastic representation of weak solutions of the Cauchy problem for SPDEs in terms of BDSDE is given. In [30] the assumption that $f$ is Lipschitz continuous in $u$ is weakened to monotonicity combined with the linear growth condition in $u$. In [3] a stochastic representation is given for SPDEs with nonlinear boundary Neumann conditions. Paper [31] also deals with stationary solutions of SPDEs and related BDSDEs with infinite horizon. In [6] the semigroup method is used to prove that if $f, g$ are Lipschitz continuous then there exists a unique mild solution of SPDE involving general non-negative self-adjoint operator $A$ (not depending on $t$ ) and finite-dimensional noise. Then this analytical result is used to get a stochastic representation of the solution in case $A$ is a diffusion operator. It is also worth noting that in $[1,9]$, in case $B$ is a space-time white noise, $A_{t}=\partial^{2} / \partial x^{2}$ and $g$ is nondegenerate, existence and uniqueness results for (1.1) with irregular $f$ are proved ( $f$ is merely measurable and satisfies some integrability condition in case $g$ is constant, or $f$ is measurable and locally bounded and $g$ is nondegenerate and satisfies some regularity conditions).

The novelty of our paper lies in the fact that we prove in a unified way the existence and uniqueness of solutions of (1.1) for much wider classes of operators then those considered in previous papers or under less restrictive assumptions on $f$ (see, however, the one dimensional results proved in $[1,9]$ ). In particular, in contrast to [6], in our paper the operators $A_{t}$ may depend on time and we only assume that $f$ is continuous and monotone in $u$ (no restrictions on the growth of $f$ with respect to $u$ ). Secondly, we show in a unified way that the solutions of (1.1) belong to some Sobolev spaces and we prove energy estimates. Moreover, we obtain stochastic representation of solutions of the form (1.5) for quasi-every (and not just $m$-a.e.) point in $E_{0, T}$. Let us stress one again, however, that except for some special cases, we have to assume that $B$ is a $Q$-Wiener process with $Q$ of trace class. Therefore our result do not cover the existence 
and uniqueness results of $[21,22]$ obtained for SPDEs with a spatially homogeneous Wiener process.

Our methods of proofs are also new. We think that of particular interest is our method for deriving from (1.5) regularity properties of $u$. The method is probabilistic in nature. As already mentioned, the general idea comes from our previous papers $[12,13,14]$ on PDEs and BSDEs. Here, however, new difficulties and subtleties arise. The idea is as follows. We show that given a solution $u$ of (1.1), i.e. a quasi-continuous function $u$ satisfying (1.5), one can find a process $M$ such that $(Y, M)=(u(\mathbf{X}), M)$ is a solution of BDSDE (1.4). Thus, in fact, (1.4) and (1.5) are equivalent. It is also worth mentioning here that the regularity of trajectories of the process $u(X)$ (i.e. the fact that $u(X)$ is càdlàg and $\left.[u(X)]_{-}=u\left(X_{-}\right)\right)$do not follow directly from the deterministic potential theory, because in general the nest for the quasi-continuous function $u(\omega ; \cdot)$ depends on $\omega \in \Omega$. From (1.4) we immediately get

$$
A_{t}^{[u]}:=u\left(\mathbf{X}_{t}\right)-u\left(\mathbf{X}_{0}\right)=M_{t}+N_{t}, \quad t \in\left[0, T_{\iota}\right]
$$

with

$$
N_{t}=-\int_{0}^{t} f\left(\mathbf{X}_{r}, u\left(\mathbf{X}_{r}\right)\right) d r-\int_{0}^{t} g\left(\mathbf{X}_{r}, u\left(\mathbf{X}_{r}\right)\right) d^{\dagger} \beta_{r}^{\iota}
$$

The process $A^{[u]}$ in (1.7) is a random additive functional (AF) of the part $\mathbf{M}^{0, T}$ of the process $\mathbf{M}$ on $E_{0, T}$. We show that $M$ is a random martingale $\mathrm{AF}$ of $\mathbf{M}^{0, T}$ of finite energy and $N$ is a random continuous $\mathrm{AF}$ of $\mathbf{M}^{0, T}$ of finite energy (we introduce these notions in Section 4). However, in most interesting cases $N$ is not of zero energy, because from (1.8) it follows that

$$
E e(N)=\frac{1}{2} E \int_{0}^{T} \int_{E}\|g(t, x, u(t, x))\|^{2} d t m(d x) .
$$

Therefore (1.7) cannot be viewed as Fukushima's decomposition of $A^{[u]}$. Nevertheless, we are able to prove the following formula for the energy of $M$ :

$$
E e(M)=E\left(\|u(0)\|_{L^{2}(E ; m)}^{2}+\int_{0}^{T} B^{(t)}(u(t), u(t)) d t-\frac{1}{2} \int_{E_{0, T}}|u(z)|^{2} k(d z)\right),
$$

where $k$ is some killing measure. Roughly speaking, we obtain energy estimate (1.6) for $u$ by combining a priori estimates for the solution $(u(\mathbf{X}), M)$ of $(1.4)$ with the estimate (1.9). The estimates for $(u(\mathbf{X}), M)$ are proved by using the methods of BSDEs. We also prove that if $\operatorname{Ee}(N)>0$ then

$$
u \notin \mathbb{W}=\left\{v \in L^{2}(\Omega \times(0, T) ; V) ; \frac{\partial v}{\partial t} \in L^{2}\left(0, T ; V^{\prime}\right) P \text {-a.s. }\right\},
$$

which shows the difference between the regularity theory for (1.1) and for usual PDEs.

In the last section of the paper we show a connection between probabilistic and mild solutions of (1.3) in case $f$ is Lipschitz continuous in $u$ and $A_{t}=A, t \in[0, T]$. Roughly speaking, changing the order of integration in (1.5) and using the fact that

$$
E_{x} f\left(X_{t}\right)=P_{t} f(x), \quad f \in L^{2}(E ; m), \quad m \text {-a.e. } x \in E,
$$


where $\left\{P_{t}, t \geq 0\right\}$ is the semigroup on $L^{2}(E ; m)$ generated by $A$, we get after some direct calculation that

$$
u(s)=P_{T-s} \varphi+\int_{s}^{T} P_{t-s} F(t, u(t)) d t+\int_{s}^{T} P_{t-s} G(t, u(t)) d^{\dagger} B_{t}
$$

where $F$ and $G$ are the Nemitskii operators corresponding to $f$ and $\tilde{g}$.

Finally, note that unlike $[12,13,14]$, in the case where $A_{t}$ are defined by $(1.2)$, in the paper we treat regularity of equations with coefficients $f, g$ depending both on the solution and its gradient.

\section{General BDSDEs}

In this section we consider general (non-Markovian) BDSDEs with final condition $\xi$ and coefficients $f, g(\operatorname{BDSDE}(\xi, f, g)$ for short) of the form

$$
Y_{t}=\xi+\int_{t}^{T} f\left(r, Y_{r}\right) d r+\int_{t}^{T} g\left(r, Y_{r}\right) d^{\dagger} \beta_{r}-\int_{t}^{T} d M_{r}, \quad t \in[0, T] .
$$

To formulate the definition of a solution of (2.1) we need some notation. In what follows $\beta=\left(\beta^{k}\right)_{k \in \mathbb{N}}$ is a sequence of mutually independent one-dimensional standard Wiener processes defined on some probability space $(\Omega, \mathcal{F}, P)$ and $\left(\Omega^{\prime}, \mathcal{G},\left(\mathcal{G}_{t}\right)_{t \in[0, T]}, P^{\prime}\right)$ is some filtered probability space such that $\left(\mathcal{G}_{t}\right)$ is right continuous and complete. We set

$$
\mathcal{F}_{t, T}^{\beta}=\sigma\left(\beta_{r}^{k}-\beta_{t}^{k}, r \in[t, T], k \in \mathbb{N}\right) \vee \mathcal{N}, \quad \mathcal{F}_{t}^{\beta}=\mathcal{F}_{0, t}^{\beta}, \quad t \in[0, T],
$$

where $\mathcal{N}=\left\{A \subset \Omega: \exists B \in \mathcal{F}_{T}^{\beta}\right.$ such that $\left.A \subset B, P(B)=0\right\}$, and then we set

$$
\mathcal{F}_{t}=\mathcal{F}_{t, T}^{\beta} \vee \mathcal{G}_{t}, \quad t \in[0, T] .
$$

Note that $\left(\mathcal{F}_{t}\right)$ is not increasing, so it is not filtration. We also set

$$
\mathbb{P}=P \otimes P^{\prime}
$$

and by $\mathbb{E}$ (resp. $E, E^{\prime}$ ) we denote the expectation with respect to the measure $\mathbb{P}$ (resp. $\left.P, P^{\prime}\right)$. Let $X$ be a process defined on $\Omega$, and $Y$ be a process on $\Omega^{\prime}$. Throughout what follows, without explicit mention we shall freely identify them with processes on $\Omega \times \Omega^{\prime}$ defined as

$$
X\left(\omega, \omega^{\prime}\right)=X(\omega), \quad Y\left(\omega, \omega^{\prime}\right)=Y\left(\omega^{\prime}\right)
$$

We will need the following spaces.

- $M$ is the space of measurable processes $X=\left\{\left(X_{t}\right)_{t \in[0, T]}\right\}$ defined on $\left(\Omega \times \Omega^{\prime}, \mathcal{F} \otimes\right.$ $\mathcal{G}, \mathbb{P})$ such that for a.e. $t \in[0, T]$ the random variable $X_{t}$ is $\mathcal{F}_{t^{-}}$measurable. $M^{2}$ is the subspace of $M$ consisting of all processes $X$ such that $\left(\mathbb{E} \int_{0}^{T}\left|X_{t}\right|^{2} d t\right)^{1 / 2}<\infty$.

- $\mathcal{S}^{2}$ is the space of càdlàg processes $Y \in M$ such that $\|Y\|_{\mathcal{S}^{2}}^{2}=\mathbb{E} \sup _{0 \leq t \leq T}\left|Y_{t}\right|^{2}<$ $\infty$. 
- $\mathcal{M}^{2}$ is the space of càdlàg processes $X \in M$ such that $X$ is an $\left(\mathcal{F}_{T}^{\beta} \vee \mathcal{G}_{t}\right)$-martingale such that $X_{0}=0$ and $\mathbb{E}[X]_{T}<\infty$, where $[X]$ denotes the quadratic variation process of $X$.

We will look for solutions of (2.1) in the space $\mathcal{S}^{2} \times \mathcal{M}^{2}$. Note that $\mathcal{S}^{2}$ equipped with the norm $\|\cdot\|_{\mathcal{S}^{2}}$ is a Banach space. Similarly, $\mathcal{M}^{2}$ equipped with the norm $\|M\|_{\mathcal{M}^{2}}=\left(\mathbb{E}[M]_{T}\right)^{1 / 2}$ is a Banach space.

Remark 2.1. By a standard argument, if $M \in \mathcal{M}^{2}$ then for $P$-a.e. $\omega \in \Omega$ the process $M(\omega, \cdot)$ is a square integrable $\left(\mathcal{G}_{t}\right)$ - martingale.

Assume that we are given an $\mathcal{F}_{T}$-measurable random variable $\xi$ and two families $\{f(t, y), t \geq 0\}_{y \in \mathbb{R}},\left\{g_{k}(t, y), t \geq 0\right\}_{y \in \mathbb{R}, k \in \mathbb{N}}$ of processes $f(\cdot, y), g_{k}(\cdot, y): \Omega \times \Omega^{\prime} \times[0, T] \rightarrow$ $\mathbb{R}$ of class $M$ (for brevity, in our notation we omit the dependence on $\left.\left(\omega, \omega^{\prime}\right) \in \Omega \times \Omega^{\prime}\right)$. Let $g(\cdot, y)=\left(g_{1}(\cdot, y), g_{2}(\cdot, y), \ldots\right)$ and let $\|x\|=\left(\sum_{k=1}^{\infty}\left|x_{k}\right|^{2}\right)^{1 / 2}$ denote the usual norm in the space $l^{2}$.

Definition. We say that a pair $(Y, M) \in \mathcal{S}^{2} \times \mathcal{M}^{2}$ is a solution of $\operatorname{BDSDE}(\xi, f, g)$ if

(a) $\int_{0}^{T}\left|f\left(t, Y_{t}\right)\right| d t<\infty, \int_{0}^{T}\left\|g\left(t, Y_{t}\right)\right\|^{2} d t<\infty \mathbb{P}$-a.s.

(b) Eq. (2.1) is satisfied P-a.s. (In (2.1), $\int_{t}^{T} g\left(r, Y_{r}\right) d^{\dagger} \beta_{r}=\sum_{k=1}^{\infty} \int_{t}^{T} g_{k}\left(r, Y_{r}\right) d^{\dagger} \beta_{r}^{k}$ and the integrals involving the processes $\beta^{k}$ are backward Itô's integrals; note that under (a) the series converges in $L^{2}(\Omega, \mathcal{F}, P)$ for $P^{\prime}$-a.s. $\left.\omega^{\prime} \in \Omega^{\prime}\right)$.

Remark 2.2. Set

$$
\hat{\beta}=\left(\hat{\beta}^{k}\right)_{k \in \mathbb{N}}, \quad \hat{\beta}_{t}^{k}=\beta_{T-t}^{k}-\beta_{T}^{k}, \quad t \in[0, T],
$$

and define $\mathcal{F}_{t, T}^{\hat{\beta}}, \mathcal{F}_{t}^{\hat{\beta}}$ to be $\mathcal{F}_{t, T}^{\beta}, \mathcal{F}_{t}^{\beta}$ but with $\beta$ replaced by $\hat{\beta}$. Then $\hat{\beta}$ is a sequence of mutually independent standard Wiener processes. If $\eta_{t}$ is $\mathcal{F}_{t, T^{-}}^{\beta}$ measurable, then $\eta_{T-t}$ is $\mathcal{F}_{t}^{\hat{\beta}}$-measurable and one can check that if $\eta=\left(\eta^{1}, \eta^{2}, \ldots\right)$ is an $\left(\mathcal{F}_{t, T}^{\beta}\right)_{t \in[0, T]^{-a d a p t e d}}$ process such that $P\left(\int_{0}^{T}\left\|\eta_{t}\right\|^{2} d t<\infty\right)=1$ then

$$
\int_{t}^{T} \eta_{s} d^{\dagger} \beta_{s}=-\int_{0}^{T-t} \eta_{T-s} d \hat{\beta}_{s}, \quad t \in[0, T]
$$

(see $[31$, p. 176]).

We are going to show that there exists a unique solution of (2.1) under the following assumptions.

(A1) $\mathbb{E}|\xi|^{2}<\infty, \mathbb{E} \int_{0}^{T}|f(t, 0)|^{2} d t+\mathbb{E} \int_{0}^{T}\|g(t, 0)\|^{2} d t<\infty$.

(A2) For every $y \in \mathbb{R}, \int_{0}^{T}|f(t, y)| d t<\infty \mathbb{P}$-a.s.

(A3) There exist constants $l, L>0$ and functions $L_{k}: \Omega \times[0, T] \rightarrow \mathbb{R}_{+}$such that $\sup _{t \leq T} \sum_{k=1}^{\infty} L_{k}^{2}(t) \leq l P$-a.s. and for a.e. $t \in[0, T]$,

(a) $\left(f(t, y)-f\left(t, y^{\prime}\right)\right)\left(y-y^{\prime}\right) \leq L\left|y-y^{\prime}\right|^{2}$ for every $y, y^{\prime} \in \mathbb{R}$, $\mathbb{P}$-a.s., 
(b) $\left|g_{k}(t, y)-g_{k}\left(t, y^{\prime}\right)\right| \leq L_{k}(t)\left|y-y^{\prime}\right|$ for for every $y, y^{\prime} \in \mathbb{R}$, $\mathbb{P}$-a.s.

(A4) For a.e. $t \in[0, T]$ the mapping $\mathbb{R} \ni y \mapsto f(t, y)$ is continuous.

The uniqueness for (2.1) follows from the following comparison result.

Proposition 2.3. Let $g$ satisfy (A3b) and either $f$ or $f^{\prime}$ satisfy (A3a). Let $(Y, M)$ be a solution of $\operatorname{BDSDE}(\xi, f, g)$ and $\left(Y^{\prime}, M^{\prime}\right)$ be a solution of $\operatorname{BSDE}\left(\xi^{\prime}, f^{\prime}, g\right)$. If $\xi \leq \xi^{\prime}$ $\mathbb{P}$-a.s. and $f^{\prime}(t, y) \leq f(t, y)$ for a.e. $t \in[0, T]$ and every $y \in \mathbb{R}$ then

$$
Y_{t}^{\prime} \leq Y_{t}, \quad t \in[0, T], \quad \mathbb{P} \text {-a.s. }
$$

Proof. We assume that $f$ satisfies (A2). In case $f^{\prime}$ satisfies (A2) the proof is analogous. By the Itô-Meyer formula,

$$
\begin{aligned}
\mid\left(Y_{t}^{\prime}\right. & \left.-Y_{t}\right)\left.^{+}\right|^{2}+\int_{t}^{T} \mathbf{1}_{\left\{Y_{r}^{\prime}>Y_{r}\right\}} d\left[M^{\prime}-M\right]_{r} \leq 2 \int_{t}^{T}\left(Y_{r}^{\prime}-Y_{r}\right)^{+}\left(f^{\prime}\left(r, Y_{r}^{\prime}\right)-f\left(r, Y_{r}\right)\right) d r \\
& +2 \int_{t}^{T}\left(Y_{r-}^{\prime}-Y_{r-}\right)^{+}\left(g\left(r, Y_{r}^{\prime}\right)-g\left(r, Y_{r}\right)\right) d^{\dagger} \beta_{r}-2 \int_{t}^{T}\left(Y_{r-}^{\prime}-Y_{r-}\right)^{+} d\left(M^{\prime}-M\right)_{r} \\
& +\sum_{k=1}^{\infty} \int_{t}^{T} \mathbf{1}_{\left\{Y_{r}^{\prime}>Y_{r}\right\}}\left|g_{k}\left(r, Y_{r}^{\prime}\right)-g_{k}\left(r, Y_{r}\right)\right|^{2} d r .
\end{aligned}
$$

By the assumptions,

$$
\int_{t}^{T}\left(Y_{r}^{\prime}-Y_{r}\right)^{+}\left(f^{\prime}\left(r, Y_{r}^{\prime}\right)-f\left(r, Y_{r}\right)\right) d r \leq L \int_{t}^{T}\left|\left(Y_{r}^{\prime}-Y_{r}\right)^{+}\right|^{2} d r
$$

and

$$
\begin{aligned}
\sum_{k=1}^{\infty} \int_{t}^{T} \mathbf{1}_{\left\{Y_{r}^{\prime}>Y_{r}\right\}}\left|g_{k}\left(r, Y_{r}^{\prime}\right)-g_{k}\left(r, Y_{r}\right)\right|^{2} d r & \leq \sum_{k=1}^{\infty} \int_{t}^{T} L_{k}^{2}(r) \mathbf{1}_{\left\{Y_{r}^{\prime}>Y_{r}\right\}}\left|Y_{r}^{\prime}-Y_{r}\right|^{2} d r \\
& \leq l \int_{t}^{T}\left|\left(Y_{r}^{\prime}-Y_{r}\right)^{+}\right|^{2} d r
\end{aligned}
$$

Hence

$$
\mathbb{E}\left|\left(Y_{t}^{\prime}-Y_{t}\right)^{+}\right|^{2} \leq 2(L+l) \mathbb{E} \int_{t}^{T}\left|\left(Y_{r}^{\prime}-Y_{r}\right)^{+}\right|^{2} d r, \quad t \in[0, T],
$$

so applying Gronwall's lemma we get the desired result.

Corollary 2.4. Let assumption (A3) hold. Then there exists at most one solution of $\operatorname{BDSDE}(\xi, f, g)$.

Proposition 2.5. Assume (A1), (A3). Let $(Y, M)$ be a solution of $\operatorname{BDSDE}(\xi, f, g)$. Then there exists $c>0$ depending only on $T, l, L$ such that

$$
\begin{aligned}
\mathbb{E}\left(\sup _{t \leq T}\left|Y_{t}\right|^{2}+\int_{0}^{T} d[M]_{t}+\right. & \left.\sup _{0 \leq t \leq T}\left|\int_{0}^{t} f\left(r, Y_{r}\right) d r\right|^{2}\right) \\
& \leq c \mathbb{E}\left(|\xi|^{2}+\int_{0}^{T}\left(|f(t, 0)|^{2}+\|g(t, 0)\|^{2}\right) d t\right) .
\end{aligned}
$$


Proof. By the Itô-Meyer formula and (A3),

$$
\begin{aligned}
\left|Y_{t}\right|^{2}+\int_{t}^{T} d[M]_{r}= & |\xi|^{2}+2 \int_{t}^{T} f\left(r, Y_{r}\right) Y_{r} d r+\int_{t}^{T}\left\|g\left(r, Y_{r}\right)\right\|^{2} d r \\
& +2 \int_{t}^{T} Y_{r} g\left(r, Y_{r}\right) d^{\dagger} \beta_{r}-2 \int_{t}^{T} Y_{r-} d M_{r} \\
\leq & |\xi|^{2}+(1+2 L+2 l) \int_{t}^{T}\left|Y_{r}\right|^{2} d r+\int_{t}^{T}\left(|f(r, 0)|^{2}+2\|g(r, 0)\|^{2}\right) d r \\
& +2 \int_{t}^{T} Y_{r} g\left(r, Y_{r}\right) d^{\dagger} \beta_{r}-2 \int_{t}^{T} Y_{r-} d M_{r}, \quad t \in[0, T], \quad \mathbb{P} \text {-a.s. }
\end{aligned}
$$

From this and Gronwall's lemma,

$$
\mathbb{E}\left|Y_{t}\right|^{2}+\mathbb{E} \int_{0}^{T} d[M]_{t} \leq c(L, l, T) \mathbb{E}\left(|\xi|^{2}+\int_{0}^{T}\left(|f(t, 0)|^{2}+\|g(t, 0)\|^{2}\right) d t\right) .
$$

Applying the Burkholder-Davis-Gundy inequality we get the desired result.

Theorem 2.6. Let assumptions (A1)-(A4) hold. Then there exists a solution $(Y, M)$ of $\operatorname{BDSDE}(\xi, f, g)$.

Proof. Step 1. Assume that $g\left(t, Y_{t}\right)=g(t), f\left(t, Y_{t}\right)=f(t), t \in[0, T]$. We first prove that there exists a solution $(Y, M)$ of the linear equation

$$
Y_{t}=\xi+\int_{t}^{T} f(r) d r+\int_{t}^{T} g(r) d^{\dagger} \beta_{r}-\int_{t}^{T} d M_{r}, \quad t \in[0, T], \quad \mathbb{P} \text {-a.s. }
$$

To this end, let us set $\mathcal{A}_{t}=\mathcal{F}_{T}^{\beta} \vee \mathcal{G}_{t}, t \in[0, T]$, and define $Y, M$ as

$$
Y_{t}=\mathbb{E}\left(\xi+\int_{t}^{T} f(r) d r+\int_{t}^{T} g(r) d^{\dagger} \beta_{r} \mid \mathcal{A}_{t}\right), \quad t \in[0, T]
$$

and

$$
\begin{aligned}
M_{t} & =\mathbb{E}\left(\xi+\int_{0}^{T} f(r) d r+\int_{0}^{T} g(r) d \beta_{r}^{\dagger} \mid \mathcal{A}_{t}\right)-Y_{0} \\
& =E\left(\xi+\int_{0}^{T} f(r) d r+\int_{0}^{T} g(r) d \beta_{r}^{\dagger} \mid \mathcal{G}_{t}\right)-Y_{0}, \quad t \in[0, T] .
\end{aligned}
$$

One can check that the pair $(Y, M)$ satisfies $(2.3)$. To show that $Y, M$ are $\left(\mathcal{F}_{t}\right)$-adapted, let us set

$$
\Lambda_{t}=\xi+\int_{t}^{T} f(r) d r+\int_{t}^{T} g(r) d^{\dagger} \beta_{r}, \quad t \in[0, T] .
$$

With this notation we have

$$
Y_{t}=\mathbb{E}\left(\Lambda_{t} \mid \mathcal{A}_{t}\right)=\mathbb{E}\left(\Lambda_{t} \mid \mathcal{G}_{t} \vee \mathcal{F}_{T}^{\beta}\right)=\mathbb{E}\left(\Lambda_{t} \mid \mathcal{G}_{t} \vee \mathcal{F}_{t, T}^{\beta} \vee \mathcal{F}_{t}^{\beta}\right)=\mathbb{E}\left(\Lambda_{t} \mid \mathcal{F}_{t} \vee \mathcal{F}_{t}^{\beta}\right)
$$


Since $f, g$ are measurable processes that are $\left(\mathcal{G}_{T} \vee \mathcal{F}_{t, T}^{\beta}\right)$-adapted, they have modifications which are $\left(\mathcal{G}_{T} \vee \mathcal{F}_{t, T}^{\beta}\right)$-progressively measurable. Therefore the integrals on the right-hand side of (2.4) are $\left(\mathcal{G}_{T} \vee \mathcal{F}_{t, T}^{\beta}\right)$-adapted. Hence

$$
\sigma\left(\Lambda_{t}\right) \subset \mathcal{G}_{T} \vee\left(\mathcal{G}_{T} \vee \mathcal{F}_{t, T}^{B}\right)=\mathcal{G}_{T} \vee \mathcal{F}_{t, T}^{\beta}
$$

It follows in particular that $\sigma\left(\Lambda_{t}\right) \vee \mathcal{F}_{t}$ is independent of $\mathcal{F}_{t}^{\beta}$. By this, [10, Proposition 5.6] and (2.5),

$$
Y_{t}=\mathbb{E}\left(\Lambda_{t} \mid \mathcal{F}_{t} \vee \mathcal{F}_{t}^{\beta}\right)=\mathbb{E}\left(\Lambda_{t} \mid \mathcal{F}_{t}\right)
$$

Thus $Y$ is $\left(\mathcal{F}_{t}\right)$-adapted. That $M$ is $\left(\mathcal{F}_{t}\right)$-adapted now follows from the equality

$$
M_{t}=Y_{t}-Y_{0}+\int_{0}^{t} f(r) d r+\int_{0}^{t} g(r) d^{\dagger} \beta_{r} .
$$

Step 2. Assume that $f$ is Lipschitz continuous in $y$ with Lipschitz constant $L$. Let $\left(Y^{0}, M^{0}\right)=(0,0)$ and let $\left(Y^{n+1}, M^{n+1}\right)$ be a solution of the equation

$$
\begin{aligned}
Y_{t}^{n+1}= & \xi+\int_{t}^{T} f\left(r, Y_{r}^{n}\right) d r+\int_{t}^{T} g\left(r, Y_{r}^{n}\right) d^{\dagger} \beta_{r} \\
& +\int_{t}^{T} d M_{r}^{n+1}, \quad t \in[0, T], \quad \mathbb{P} \text {-a.s. }
\end{aligned}
$$

The sequence $\left\{\left(Y^{n}, M^{n}\right)\right\}$ is well defined, i.e. if $\left(Y^{n}, M^{n}\right) \in \mathcal{S}^{2} \otimes \mathcal{M}^{2}$ then $\left(Y^{n+1}, M^{n+1}\right)$ exists and belongs to $\mathcal{S}^{2} \otimes \mathcal{M}^{2}$. To see this, assume that $\left(Y^{n}, M^{n}\right) \in \mathcal{S}^{2} \otimes \mathcal{M}^{2}$. Then

$$
\begin{aligned}
& \mathbb{E} \int_{0}^{T}\left|f\left(t, Y_{t}^{n}\right)\right|^{2} d r \leq 2 L^{2} \mathbb{E} \int_{0}^{T}\left|Y_{t}^{n}\right|^{2} d t+2 \mathbb{E} \int_{0}^{T}|f(t, 0)|^{2} d t<\infty, \\
& \mathbb{E} \int_{0}^{T}\left\|g\left(t, Y_{t}^{n}\right)\right\|^{2} d t \leq 2 L^{2} \mathbb{E} \int_{0}^{T}\left|Y_{t}^{n}\right|^{2} d t+2 \mathbb{E} \int_{0}^{T}\|g(t, 0)\|^{2} d t<\infty .
\end{aligned}
$$

Therefore by Step 1 there exists a solution $\left(Y^{n+1}, M^{n+1}\right) \in \mathcal{S}^{2} \otimes \mathcal{M}^{2}$ of (2.6). By the Itô-Meyer formula and the assumption on $f$,

$$
\begin{aligned}
\mid Y_{t}^{n+1} & -\left.Y_{t}^{n}\right|^{2}+\int_{t}^{T} d\left[M^{n+1}-M^{n}\right]_{r} \\
= & 2 \int_{t}^{T}\left(Y_{r}^{n+1}-Y_{r}^{n}\right)\left(f\left(r, Y_{r}^{n}\right)-f\left(r, Y_{r}^{n-1}\right)\right) d r \\
& -2 \int_{t}^{T}\left(Y_{r-}^{n+1}-Y_{r-}^{n}\right) d\left(M^{n+1}-M^{n}\right)_{r} \\
& +2 \int_{t}^{T}\left(Y_{r}^{n+1}-Y_{r}^{n}\right)\left(g\left(r, Y_{r}^{n}\right)-g\left(r, Y_{r}^{n-1}\right)\right) d^{\dagger} \beta_{r} \\
& +\int_{t}^{T}\left\|g\left(r, Y_{r}^{n}\right)-g\left(r, Y_{r}^{n-1}\right)\right\|^{2} d r \\
\leq & \int_{t}^{T}\left|Y_{r}^{n+1}-Y_{r}^{n}\right|^{2} d r+L^{2} \int_{t}^{T}\left|Y_{t}^{n}-Y_{r}^{n-1}\right|^{2} d r \\
& +l^{2} \int_{t}^{T}\left|Y_{r}^{n}-Y_{r}^{n-1}\right|^{2} d r-2 \int_{t}^{T}\left(Y_{r-}^{n+1}-Y_{r-}^{n}\right) d\left(M^{n+1}-M^{n}\right)_{r} \\
& -2 \int_{t}^{T}\left(Y_{r}^{n+1}-Y_{r}^{n}\right)\left(g\left(r, Y_{r}^{n}\right)-g\left(r, Y_{r}^{n-1}\right)\right) d^{\dagger} \beta_{r} .
\end{aligned}
$$


Taking the expectation of both sides of the above inequality and using Gronwall's lemma we get

$$
\mathbb{E}\left|Y_{t}^{n+1}-Y_{t}^{n}\right|^{2}+\mathbb{E} \int_{t}^{T} d\left[M^{n+1}-M^{n}\right]_{r} \leq C \mathbb{E} \int_{t}^{T}\left|Y_{r}^{n}-Y_{t}^{n-1}\right|^{2} d r, \quad t \in[0, T]
$$

for some $C$ depending only on $T, L, l$. Hence

$$
\sup _{t \leq r \leq T} \mathbb{E}\left|Y_{r}^{n+1}-Y_{r}^{n}\right|^{2}+\mathbb{E} \int_{t}^{T} d\left[M^{n+1}-M^{n}\right]_{r} \leq C(T-t) \sup _{t \leq r \leq T} \mathbb{E}\left|Y_{r}^{n}-Y_{r}^{n-1}\right|^{2} .
$$

Write

$$
\|(Y, M)\|_{t}^{2}=\sup _{t \leq r \leq T} \mathbb{E}\left|Y_{r}\right|^{2}+\mathbb{E} \int_{t}^{T} d[M]_{r}
$$

With this notation we have

$$
\begin{aligned}
& \left\|\left(Y^{n+1}, M^{n+1}\right)-\left(Y^{n}, M^{n}\right)\right\|_{t} \\
& \quad \leq C(T-t)^{1 / 2}\left\|\left(Y^{n}, M^{n}\right)-\left(Y^{n-1}-M^{n-1}\right)\right\|_{t}, \quad n \geq 1, \quad t \in[0, T]
\end{aligned}
$$

From this one can deduce that

$$
\left\|\left(Y^{n+k}, M^{n+k}\right)-\left(Y^{n}, M^{n}\right)\right\|_{t_{1}} \leq 2^{-(n+1)}\left\|\left(Y^{1}, M^{1}\right)\right\|_{t_{1}}
$$

for $t_{1} \in[0, T]$ such that $C\left(T-t_{1}\right)=2^{-1}$. Therefore dividing the interval $[0, T]$ into small intervals and using a standard argument one can show that

$$
\lim _{n, m \rightarrow \infty}\left\|\left(Y^{m}, M^{m}\right)-\left(Y^{n}-M^{n}\right)\right\|_{0}=0 .
$$

Using the Burkholder-Davis-Gundy inequality we conclude from the above convergence and (2.7) with $\left(Y^{n+1}, M^{n+1}\right)$ replaced by $\left(Y^{m}, M^{m}\right)$ that

$$
\lim _{n, m \rightarrow \infty} \mathbb{E}\left(\sup _{0 \leq t \leq T}\left|Y_{t}^{n}-Y_{t}^{m}\right|^{2}+\int_{0}^{T} d\left[M^{n}-M^{m}\right]_{t}\right)=0 .
$$

Let $(Y, M)$ be the limit of $\left\{\left(Y^{n}, M^{n}\right)\right\}$ in $\mathcal{S}^{2} \times \mathcal{M}^{2}$. Letting $n \rightarrow \infty$ in (2.6) shows that $(Y, M)$ is a solution of $\operatorname{BDSDE}(\xi, f, g)$.

Step 3. Now we assume that $f$ satisfies the assumptions of the theorem and moreover there exists $\lambda \in \mathbb{R}$ such that $f(t, y) \geq \lambda$ for a.e. $t \in[0, T]$ and every $y \in \mathbb{R}$. Put

$$
f_{n}(t, y)=\inf _{x \in \mathbb{Q}}\{n|y-x|+f(t, x)-L x\}+L y, \quad t \in[0, T], \quad y \in \mathbb{R} .
$$

It is an elementary check that $f_{n}$ has the following properties: for a.e. $t \in[0, T]$,

(a) $\left|f_{n}(t, y)-f_{n}\left(t, y^{\prime}\right)\right| \leq(L+n)\left|y-y^{\prime}\right|$ for all $y, y^{\prime} \in \mathbb{R}$,

(b) $\lambda \leq f_{n}(t, y) \leq f(t, y)$ for every $y \in \mathbb{R}$,

(c) $f_{n}(t, \cdot) \nearrow f(t, \cdot)$ uniformly on compact subsets of $\mathbb{R}$,

(d) $\left(f_{n}(t, y)-f_{n}\left(t, y^{\prime}\right)\right)\left(y-y^{\prime}\right) \leq L\left|y-y^{\prime}\right|^{2}$ for all $y, y^{\prime} \in \mathbb{R}$. 
By Step 2, for each $n \geq 1$ there is a solution $\left(Y^{n}, M^{n}\right) \in \mathcal{S}^{2} \otimes \mathcal{M}^{2}$ of $\operatorname{BDSDE}\left(\xi, f_{n}, g\right)$. By Proposition 2.3,

$$
Y_{t}^{n} \leq Y_{t}^{n+1}, \quad t \in[0, T], \quad \mathbb{P} \text {-a.s., } \quad n \geq 1 .
$$

Put $Y_{t}=\sup _{n \geq 1} Y_{t}^{n}$. By Proposition 2.5 and (b),

$$
\begin{aligned}
\mathbb{E} \sup _{t \leq T}\left|Y_{t}^{n}\right|^{2}+\mathbb{E}\left[M^{n}\right]_{T} & \leq C \mathbb{E}\left(|\xi|^{2}+\int_{0}^{T}\left(\left|f_{n}(t, 0)\right|^{2}+\|g(t, 0)\|^{2}\right) d t\right) \\
& \leq C \mathbb{E}\left(|\xi|^{2}+\lambda^{2}+\int_{0}^{T}\left(|f(t, 0)|^{2}+\|g(t, 0)\|^{2}\right) d t\right) .
\end{aligned}
$$

For every $\varepsilon, \eta>0$ we have

$$
\begin{aligned}
& \mathbb{P}(\left.\int_{0}^{T}\left|f_{n}\left(t, Y_{t}^{n}\right)-f\left(t, Y_{t}\right)\right| d t>\varepsilon\right) \\
& \leq \mathbb{P}\left(\int_{0}^{T}\left|f_{n}\left(t, Y_{t}^{n}\right)-f\left(t, Y_{t}\right)\right| d t>\varepsilon, \sup _{n \geq 1} \sup _{t \leq T}\left|Y_{t}^{n}\right| \leq \eta\right)+\mathbb{P}\left(\sup _{n \geq 1} \sup _{t \leq T}\left|Y_{t}^{n}\right| \geq \eta\right) \\
& \quad \leq \mathbb{P}\left(\int_{0}^{T}\left|f_{n}\left(t, Y_{t}^{n}\right)-f\left(t, Y_{t}\right)\right| d t>\varepsilon, \sup _{n \geq 1} \sup _{t \leq T}\left|Y_{t}^{n}\right| \leq \eta\right) \\
& \quad+\eta^{-2}\left(\mathbb{E} \sup _{t \leq T}\left|Y_{t}^{1}\right|^{2}+\mathbb{E} \sup _{t \leq T}\left|Y_{t}\right|^{2}\right),
\end{aligned}
$$

the last inequality being a consequence of (2.8) and Chebyshev's inequality. By (b), (c) and (A2) the first term on the right-hand side of the above inequality tends to zero as $n \rightarrow \infty$. The second one tends to zero as $\eta \rightarrow \infty$ thanks to (2.9). Hence

$$
\sup _{0 \leq t \leq T}\left|\int_{0}^{t}\left(f_{n}\left(r, Y_{r}^{n}\right)-f\left(r, Y_{r}\right)\right) d r\right| \rightarrow 0
$$

in probability $\mathbb{P}$ as $n \rightarrow \infty$. By (2.8), (2.9) and (A3) we also have

$$
\begin{aligned}
\mathbb{E} \sup _{0 \leq t \leq T}\left|\int_{0}^{t}\left(g\left(r, Y_{r}^{n}\right)-g(r, Y)\right) d^{\dagger} \beta_{r}\right|^{2} & \leq 4 \mathbb{E} \sum_{k=1}^{\infty} \int_{0}^{T}\left|g_{k}\left(t, Y_{t}^{n}\right)-g_{k}\left(t, Y_{t}\right)\right|^{2} d t \\
& \leq 4 l \mathbb{E} \int_{0}^{T}\left|Y_{t}^{n}-Y_{t}\right|^{2} d t,
\end{aligned}
$$

which converges to zero as $n \rightarrow \infty$. By Proposition 2.5,

$$
\sup _{n \geq 1} \mathbb{E} \sup _{0 \leq t \leq T}\left|\int_{0}^{t} f_{n}\left(r, Y_{r}^{n}\right) d r\right|^{2}<\infty .
$$

Therefore letting $n \rightarrow \infty$ in the equation

$$
Y_{t}^{n}=\mathbb{E}\left(\xi+\int_{t}^{T} f_{n}\left(r, Y_{r}^{n}\right) d r+\int_{t}^{T} g\left(r, Y_{r}^{n}\right) d^{\dagger} \beta_{r} \mid \mathcal{F}_{t}\right), \quad t \in[0, T]
$$

shows that $(Y, M)$, where

$$
M_{t}=\mathbb{E}\left(\xi+\int_{0}^{T} f\left(r, Y_{r}\right) d r+\int_{0}^{T} g\left(r, Y_{r}\right) d^{\dagger} \beta_{r} \mid \mathcal{F}_{t}\right)-Y_{0},
$$


is a solution of $\operatorname{BDSDE}(\xi, f, g)$.

Step 4. We now show how to dispense with the assumption that $f$ is bounded from below. Let $f_{n}=f \vee(-n)$. By Step 3, for each $n \geq 1$ there exists a solution $\left(Y^{n}, M^{n}\right)$ of $\operatorname{BDSDE}\left(\xi, f_{n}, g\right)$. By Proposition 2.3, $Y_{t}^{n} \geq Y_{t}^{n+1}, t \in[0, T]$, P-a.s. for $n \geq 1$, whereas by Proposition 2.5,

$$
\mathbb{E} \sup _{t \leq T}\left|Y_{t}^{n}\right|^{2}+\mathbb{E} \int_{0}^{T} d\left[M^{n}\right]_{t} \leq C \mathbb{E}\left(|\xi|^{2}+\int_{0}^{T}\left(|f(t, 0)|^{2}+\|g(t, 0)\|^{2}\right) d t\right) .
$$

Using the above properties of the processes $Y^{n}$ one can show in much the same way as in Step 3 that $(2.10),(2.11)$ hold true and then that there exists a solution $(Y, M)$ of $\operatorname{BDSDE}(\xi, f, g)$.

\section{SPDEs and Markov-type BDSDEs}

In this section we first consider Markov-type BDSDEs. Roughly speaking, these are BDSDEs of the form (2.1) with filtration $\left(\mathcal{G}_{t}\right)$ generated by some Markov process $\mathbf{M}=$ $\left(\mathbf{X}, P_{z}\right)$ and with final condition $\xi$ and coefficients $f, g$ depending on $\omega^{\prime}$ only through $\mathbf{X}\left(\omega^{\prime}\right)$. In our paper $\mathbf{M}$ is a Markov process associated with a time-dependent Dirichlet form. Using results of Section 3 we prove the existence and uniqueness of solutions of BDSDEs associated with $\mathbf{M}$ for $\xi, f, g$ satisfying some "markovian" analogue of conditions (A1)-(A4). Then we use this result to prove the existence and uniqueness of solutions of SPDE of the form (1.1) with operator $\frac{\partial}{\partial t}+A_{t}$ associated with the underlying Dirichlet form.

\subsection{Dirichlet forms and Markov processes}

In what follows, $E$ is a locally compact separable metric space and $m$ is an everywhere dense Radon measure on $E$. By $\Delta$ we denote the one-point compactification of $E$. If $E$ is already compact then we adjoin $\Delta$ to $E$ as an isolated point. We set $E^{1}=\mathbb{R} \times E$, $E_{T}=[0, T] \times E, E_{0, T}=(0, T] \times E, m^{1}=l^{1} \otimes m, m_{T}=\left.l^{1}\right|_{[0, T]} \otimes m$, where $l^{1}$ is the one-dimensional Lebesgue measure. We adopt the convention that every function $\varphi$ on $E$ is extended to $E^{1}$ by putting $\varphi(t, x)=\varphi(x),(t, x) \in E^{1}$, and every function $f$ on $E^{1}$ is extended to $E^{1} \cup\{\Delta\}$ by putting $f(\Delta)=0$. Similarly, we extend functions defined on $E_{0, T}$ or on $E_{T}$ to functions on $E^{1} \cup\{\Delta\}$ by putting $f(z)=0$ outside $E_{0, T}$ or $E_{T}$, respectively.

We assume that we are given a family $\left\{B^{(t)}, t \in \mathbb{R}\right\}$ of regular Dirichlet forms on $H=L^{2}(E ; m)$ with sector constant independent of $t$ and common domain $V \subset H$ (see, e.g., $[16,27]$ for the definition). We also assume that

(a) $\mathbb{R} \ni t \mapsto B^{(t)}(\varphi, \psi)$ is measurable for every $\varphi, \psi \in V$,

(b) there exist $c_{1}, c_{2}>0$ such that $c_{1} B^{(0)}(\varphi, \varphi) \leq B^{(t)}(\varphi, \varphi) \leq c_{2} B^{(0)}(\varphi, \varphi)$ for every $t \in \mathbb{R}$ and $\varphi \in V$.

By assumption, $\left(B^{(0)}, V\right)$ is closed, i.e. $V$ is a real Hilbert space with respect to $\tilde{B}_{1}^{(0)}(\cdot, \cdot)$, where $\tilde{B}^{(0)}(\varphi, \psi)=\frac{1}{2}\left(B^{(0)}(\varphi, \psi)+B^{(0)}(\psi, \varphi)\right)$ and $\tilde{\mathcal{B}}_{1}^{(0)}(\varphi, \psi)=\tilde{\mathcal{B}}^{(0)}(\varphi, \psi)+(\varphi, \psi)_{H}$ for $\varphi, \psi \in V$. By $V^{\prime}$ we denote the dual space of $V$ and we set

$$
\mathcal{V}=L^{2}(\mathbb{R} ; V), \quad \mathcal{V}^{\prime}=L^{2}\left(\mathbb{R} ; V^{\prime}\right), \quad \mathcal{W}=\left\{u \in \mathcal{V}: \frac{\partial u}{\partial t} \in \mathcal{V}^{\prime}\right\}
$$


We will consider two time dependent Dirichlet forms $(\mathcal{E}, D(\mathcal{E}))$ and $\left(\mathcal{E}^{0, T}, D\left(\mathcal{E}^{0, T}\right)\right)$ associated with the families $\left\{B^{(t)}, t \in \mathbb{R}\right\}$ and $\left\{B^{(t)}, t \in[0, T]\right\}$, respectively. The first one we define by putting $D(\mathcal{E})=(\mathcal{W} \otimes \mathcal{V}) \cup(\mathcal{V} \otimes \mathcal{W})$ and

$$
\mathcal{E}(u, v)=\left\{\begin{array}{l}
\left\langle\frac{\partial u}{\partial t}, v\right\rangle+\int_{\mathbb{R}} B^{(t)}(u(t), v(t)) d t, \quad u \in \mathcal{W}, v \in \mathcal{V}, \\
-\left\langle u, \frac{\partial v}{\partial t}\right\rangle+\int_{\mathbb{R}} B^{(t)}(u(t), v(t)) d t, \quad u \in \mathcal{V}, v \in \mathcal{W},
\end{array}\right.
$$

where $\langle\cdot, \cdot\rangle$ denotes the duality pairing between $\mathcal{V}$ and $\mathcal{V}^{\prime}$. It is known (see [27, Example I.4.9]) that $(\mathcal{E}, D(\mathcal{E}))$ is a generalized Dirichlet form on $L^{2}\left(E^{1} ; m^{1}\right)$.

By [17, Theorem 4.2] (see also [19, Theorem 6.3.1]) there exists a Hunt process $\mathbf{M}=\left(\Omega^{\prime},\left(\mathbf{X}_{t}\right)_{t \geq 0},\left(P_{z}\right)_{z \in E^{1} \cup \Delta},\left(\mathcal{F}_{t}^{\mathbf{X}}\right)_{t \geq 0}\right)$ with state space $E^{1}$, life time $\zeta$ and cemetery state $\Delta$ properly associated with $(\mathcal{E}, D(\mathcal{E}))$ in the resolvent sense. Moreover,

$$
\mathbf{X}_{t}=\left(\iota(t), X_{\iota(t)}\right), \quad t \geq 0
$$

where $\iota$ is the uniform motion to the right, i.e. $\iota(t)=\iota(0)+t, \iota(0)=s, P_{z}$-a.s. for $z=$ $(s, x)$. One can also check that $\left(X,\left(P_{s, x}\right)_{x \in E}\right)$ is a time inhomogeneous Markov process associated with the family $\left\{\left(B^{(t)}, V\right), t \geq 0\right\}$, i.e. for every $s \in \mathbb{R},\left(X_{s+.},\left(P_{s, x}\right)_{x \in E}\right)$ is a Hunt process associated with the form $\left(B^{(s)}, V\right)$.

Let

$$
\begin{aligned}
& \mathcal{W}_{T}=\left\{u \in L^{2}(0, T ; V): \frac{\partial u}{\partial t} \in L^{2}\left(0, T ; V^{\prime}\right), u(T)=0\right\}, \\
& \mathcal{W}_{0}=\left\{u \in L^{2}(0, T ; V): \frac{\partial u}{\partial t} \in L^{2}\left(0, T ; V^{\prime}\right), u(0)=0\right\} .
\end{aligned}
$$

We define the second form by setting $D\left(\mathcal{E}^{0, T}\right)=\left(\mathcal{W}_{T} \otimes L^{2}(0, T ; V)\right) \cup\left(\mathcal{W}_{0} \otimes L^{2}(0, T ; V)\right)$ and

$$
\mathcal{E}^{0, T}(u, v)=\left\{\begin{array}{l}
\left\langle\frac{\partial u}{\partial t}, v\right\rangle+\mathcal{B}^{0, T}(u, v), \quad u \in \mathcal{W}_{0}, v \in L^{2}(0, T ; V), \\
-\left\langle u, \frac{\partial v}{\partial t}\right\rangle+\mathcal{B}^{0, T}(u, v), \quad v \in \mathcal{W}_{T}, u \in L^{2}(0, T ; V),
\end{array}\right.
$$

where now $\langle\cdot, \cdot\rangle$ denotes the duality pairing between $L^{2}\left(0, T ; V^{\prime}\right)$ and $L^{2}(0, T ; V)$, and

$$
\mathcal{B}^{0, T}(u, v)=\int_{0}^{T} B^{(t)}(u(t), v(t)) d t, \quad u, v \in L^{2}(0, T ; V) .
$$

Note that by [27, Example I.4.9], $\left(\mathcal{E}^{0, T}, D\left(\mathcal{E}^{0, T}\right)\right)$ is a generalized Dirichlet form on $L^{2}\left(E_{0, T} ; m_{T}\right)$.

We set

$$
\begin{gathered}
R_{\alpha} f(z)=E_{z} \int_{0}^{\infty} e^{-\alpha t} f\left(\mathbf{X}_{t}\right) d t, \quad z \in E^{1}, \quad f \in \mathcal{B}\left(E^{1}\right), \quad \alpha \geq 0, \\
R_{\alpha}^{0, T} f(z)=E_{z} \int_{0}^{T-\iota(0)} e^{-\alpha t} f\left(\mathbf{X}_{t}\right) d t, \quad z \in E_{0, T}, \quad f \in \mathcal{B}\left(E_{0, T}\right), \quad \alpha \geq 0
\end{gathered}
$$

whenever the integrals exist. Let $\hat{\mathbf{M}}=\left(\hat{\mathbf{X}}, \hat{P}_{z}\right)$ be the dual process of $\mathbf{M}$ (see [19, Section 3.3]). We set (whenever the integrals exist)

$$
\hat{R}_{\alpha} f(z)=\hat{E}_{z} \int_{0}^{\infty} e^{-\alpha t} f\left(\hat{\mathbf{X}}_{t}\right) d t, \quad z \in E^{1}, \quad f \in \mathcal{B}\left(E^{1}\right), \quad \alpha \geq 0,
$$




$$
\hat{R}_{\alpha}^{0, T} f(z)=\hat{E}_{z} \int_{0}^{\iota(0)} e^{-\alpha t} f\left(\hat{\mathbf{X}}_{t}\right) d t, \quad z \in E_{0, T}, \quad f \in \mathcal{B}\left(E_{0, T}\right), \quad \alpha \geq 0 .
$$

To shorten notation, we write $R=R_{0}, \hat{R}=\hat{R}_{0}, R^{0, T}=R_{0}^{0, T}, \hat{R}^{0, T}=\hat{R}_{0}^{0, T}$. It is well known that $G_{\alpha}=R_{\alpha}, \hat{G}_{\alpha}=\hat{R}_{\alpha}$ on $L^{2}\left(E^{1} ; m^{1}\right), \alpha>0$, where $\left\{G_{\alpha}, \alpha>0\right\}$ is the resolvent and $\left\{\hat{G}_{\alpha}, \alpha>0\right\}$ is the dual resolvent associated with the form $(\mathcal{E}, D(\mathcal{E}))$. Similarly, $G_{\alpha}^{0, T}=R_{\alpha}^{0, T}, \hat{G}_{\alpha}^{0, T}=\hat{R}_{\alpha}^{0, T}$ on $L^{2}\left(E_{0, T} ; m_{T}\right), \alpha \geq 0$, where $\left\{G_{\alpha}^{0, T}, \alpha \geq 0\right\}$ is the resolvent and $\left\{\hat{G}_{\alpha}^{0, T}, \alpha \geq 0\right\}$ is the dual resolvent associated with $\left(\mathcal{E}^{0, T}, D\left(\mathcal{E}^{0, T}\right)\right)$.

By $A_{t}$ we denote the operator associated with the form $\left(B^{(t)}, V\right)$, i.e.

$$
\left(-A_{t} u, v\right)=B^{(t)}(u, v), \quad u \in D\left(A_{t}\right), v \in V,
$$

where $D\left(A_{t}\right)=\left\{u \in V: v \mapsto B^{(t)}(u, v)\right.$ is continuous with respect to $(\cdot, \cdot)^{1 / 2}$ on $\left.V\right\}$ (see [16, Proposition I.2.16]).

Let cap be the capacity considered in [18] (see also [19, Section 6.2]). We say that a set $B$ is $\mathcal{E}$-exceptional if $\operatorname{cap}(B)=0$. We say that some property is satisfied quasieverywhere (q.e. for short) if the set of those $z \in E^{1}$ for which it does not hold is $\mathcal{E}$-exceptional. Note that a nearly Borel set $B$ is $\mathcal{E}$-exceptional if and only if it is $\mathbf{M}$ exceptional, i.e. $P_{m_{1}}\left(\sigma_{B}<\infty\right)=0$, where $\sigma_{B}=\inf \left\{t>0: \mathbf{X}_{t} \in B\right\}$ (see, e.g., [18, p. 298]).

We say that $f: E_{0, T} \rightarrow \mathbb{R}$ is quasi-integrable $\left(f \in q L^{1}\right.$ in notation) if $f$ is Borel measurable and $P_{z}\left(\int_{0}^{T-\iota(0)}\left|f\left(\mathbf{X}_{t}\right)\right| d t<\infty\right)=1$ for q.e. $z \in E_{0, T}$. By $q \mathbb{L}^{1}$ we denote the set of measurable functions $f: \Omega \times E_{0, T} \rightarrow \mathbb{R}$ such that $P\left(\left\{\omega \in \Omega ; f(\omega, \cdot) \in q L^{1}\right\}\right)=1$.

\subsection{Existence and uniqueness of solutions}

Let $A_{t}$ be the operator defined by (3.3). Suppose we are given measurable functions $\varphi: E \rightarrow \mathbb{R}, f: \Omega \times E_{T} \times \mathbb{R} \rightarrow \mathbb{R}, g_{k}: \Omega \times E_{T} \times \mathbb{R} \rightarrow \mathbb{R}, k \in \mathbb{N}$, and let $g=\left(g_{1}, g_{2}, \ldots\right)$. We are going to show that there exists a unique solution of the SPDE

$$
d u(t)=-\left(A_{t} u+f(t, x, u)\right) d t-g(t, x, u) d^{\dagger} \beta_{t}, \quad u(T)=\varphi,
$$

under hypotheses (H1)-(H5) given below:

$$
\|\varphi\|_{L^{2}(E ; m)}+E\|f(\cdot, 0)\|_{L^{2}\left(E_{0, T} ; m_{T}\right)}^{2}+E \sum_{k=1}^{\infty}\left\|g_{k}(\cdot, 0)\right\|_{L^{2}\left(E_{0, T} ; m_{T}\right)}^{2}<\infty .
$$

(H2) For every $y \in \mathbb{R}$ the mapping $E_{0, T} \ni z \mapsto f(z, y)$ belongs to $q \mathbb{L}^{1}$.

(H3) There exist $l, L>0$ and measurable functions $L_{k}: E_{0, T} \rightarrow[0, \infty)$ such that $\sup _{z \in E_{0, T}} \sum_{k=1}^{\infty} L_{k}^{2}(z) \leq l$ and for every $z \in E_{0, T}$ and $y, y^{\prime} \in \mathbb{R}$,

(a) $\left(f(z, y)-f\left(z, y^{\prime}\right)\right)\left(y-y^{\prime}\right) \leq L\left|y-y^{\prime}\right|^{2}$,

(b) $\left|g_{k}(z, y)-g_{k}\left(z, y^{\prime}\right)\right| \leq L_{k}(z)\left|y-y^{\prime}\right|$.

(H4) For every $\omega \in \Omega$ and $z \in E_{0, T}$ the mapping $\mathbb{R} \ni y \mapsto f(z, y)$ is continuous.

(H5) For every $y \in \mathbb{R}$ the mappings $f(\cdot, y), g_{k}(\cdot, y): \Omega \times E_{T} \rightarrow \mathbb{R}, k \in \mathbb{N}$, are $\left(\mathcal{F}_{t, T}^{\beta}\right)$ progressively measurable, i.e. for every $t \in[0, T]$ the mappings $\Omega \times[T-t, T] \times E \ni$ $(\omega, s, x) \mapsto f(\omega, s, x, y)$ and $\Omega \times[T-t, T] \times E \ni(\omega, s, x) \mapsto g_{k}(\omega, s, x, y)$ are $\mathcal{F}_{t, T}^{\beta} \otimes \mathcal{B}([T-t, T]) \otimes \mathcal{B}(E)$-measurable. 
In what follows

$$
\beta_{t}^{\iota}=\beta_{t+\iota(0)}, \quad T_{\iota}=T-\iota(0), \quad \mathcal{F}_{t}=\mathcal{F}_{t, T_{\iota}}^{\beta^{\iota}} \vee \mathcal{F}_{t}^{\mathbf{X}}, \quad t \in[0, T],
$$

where $\mathcal{F}_{t, T_{\iota}}^{\beta^{\iota}}=\sigma\left(\beta_{r \wedge T_{\iota}}^{\iota}-\beta_{t}^{\iota}, r \in[t, T]\right)$ (see our convention (2.2)).

We will say that a random function $u: \Omega \times E_{0, T} \rightarrow \mathbb{R}$ is adapted if $u(\mathbf{X}) \in M$, where $M$ is defined as in Section 2 but with respect to $\left(\mathcal{F}_{t}\right)$ defined by (3.5).

Remark 3.1. (i) Assume (H5). Then for every $y \in \mathbb{R}$ there exists a $P \otimes m_{T}$-version $\tilde{f}(\cdot, y)$ of $f(\cdot, y)$ and a $P \otimes m_{T}$-version $\tilde{g}_{k}(\cdot, y)$ of $g_{k}(\cdot, y)$ such that the processes $\tilde{f}(\mathbf{X}, y), \tilde{g}_{k}(\mathbf{X}, y)$ are of class $M$ under $\mathbb{P}_{z}$ for q.e. $z \in E_{0, T}$. To see this, we let $h$ stand for $f(\cdot, y)$ or for $g_{k}(\cdot, y)$. It is known that any $\left(\mathcal{F}_{t, T}^{\beta}\right)$-progressively measurable square-integrable $h: \Omega \times E_{T} \rightarrow \mathbb{R}$ may be approximated in the space $L^{2}\left(\Omega \times E_{T} ; P \otimes m_{T}\right)$ by linear combinations of processes of the form

$$
S_{j}(\omega, t, x)=\mathbf{1}_{\Lambda_{j}}(\omega) \mathbf{1}_{\left(T-r_{j+1}, T-r_{j}\right]}(t) b_{j}(x),
$$

where $0 \leq r_{j} \leq r_{j+1} \leq T, \Lambda_{j} \in \mathcal{F}_{T-r_{j}, T}^{\beta}$ and $\left\{b_{j}, j \geq 0\right\}$ is some orthonormal basis of $H$. It is an elementary check that the process $\left\{S_{j}\left(\mathbf{X}_{t}\right), t \geq 0\right\}$ is $\left\{\mathcal{F}_{t, T_{\iota}}^{\beta^{\iota}}\right\}$-adapted. Therefore linear combinations of processes of the form (3.6) are of class $M$. Hence there is a sequence $\left\{h_{n}\right\}$ such that $h_{n} \rightarrow h$ in $L^{2}\left(\Omega \times E_{0, T} ; P \otimes m_{T}\right)$ and the processes $h_{n}(\mathbf{X})$ are of class $M$. Let $\tilde{h}=\limsup _{n \rightarrow \infty} h_{n}$. Then $\tilde{h}(\mathbf{X})$ is of class $M$ and $\tilde{h}$ is a $P \otimes m_{T}$-version of $h$. Let $\hat{S}_{00}$ denote the set of all finite zero order integral measures $\nu$ on $E_{0, T}$ such that $\nu\left(E_{0, T}\right)<\infty$ and $\left\|\hat{R}^{0, T} \nu\right\|_{\infty}<\infty$. For every $\nu \in \hat{S}_{00}$ we have

$$
\mathbb{E}_{\nu} \int_{0}^{T_{\iota}} \mid\left(\tilde{h}\left(\mathbf{X}_{t}\right)-\left.h\left(\mathbf{X}_{t}\right)\right|^{2} d t \leq E\|\tilde{h}-h\|_{L^{2}\left(E_{0, T} ; m_{T}\right)}^{2}\left\|\hat{R}^{0, T} \nu\right\|_{\infty}=0 .\right.
$$

From this and [28, Theorem 2.5] (or remark at the end of Section 4 in [18]) it follows that $\mathbb{P}_{z}\left(\tilde{h}\left(\mathbf{X}_{t}\right)=h\left(\mathbf{X}_{t}\right)\right.$ for a.e. $\left.t \in[0, T]\right)=1$ for q.e. $z \in E_{0, T}$, which shows the desired result in case $h$ is square-integrable. The general case is handled by approximating $h$ pointwise by square-integrable functions.

(ii) From the fact that Carathéodory functions are jointly measurable it follows that if $f, g_{k}$ satisfy (H3b), (H4), (H5) and $u: \Omega \times E_{0, T} \rightarrow \mathbb{R}$ is adapted then the processes $t \mapsto \tilde{f}\left(\mathbf{X}_{t}, u\left(\mathbf{X}_{t}\right)\right), t \mapsto \tilde{g}_{k}\left(\mathbf{X}_{t}, u\left(\mathbf{X}_{t}\right)\right)$ are of class $M$.

We first give the definition of a solution of (3.4) and related Markov-type BDSDE with coefficients $f, g$. In what follows we always assume that the coefficients $f, g_{k}, k \geq$ 1, satisfy (H5), and we always assume that we are taking their versions having the properties listed in Remark 3.1.

Definition. We say that an adapted function $u: \Omega \times E_{0, T} \rightarrow \mathbb{R}$ is a solution of SPDE (3.4) if

(a) $\mathbb{P}_{z}\left(\int_{0}^{T_{\iota}}\left|f\left(\mathbf{X}_{t}, u\left(\mathbf{X}_{t}\right)\right)\right| d t<\infty\right)=1, \mathbb{E}_{z} \sup _{0 \leq t \leq T_{\iota}}\left|\int_{0}^{t} f\left(\mathbf{X}_{r}, u\left(\mathbf{X}_{r}\right)\right) d r\right|^{2}<\infty$ and $\mathbb{E}_{z} \int_{0}^{T_{\iota}}\left\|g\left(\mathbf{X}_{t}, u\left(\mathbf{X}_{t}\right)\right)\right\|^{2} d t<\infty$ for q.e. $z \in E_{0, T}$, where $\mathbb{P}_{z}=P \otimes P_{z}$ and $\mathbb{E}_{z}$ denotes the expectation with respect to $\mathbb{P}_{z}$, 
(b) for $P$-a.e. $\omega \in \Omega, u(\omega ; \cdot): E_{0, T} \rightarrow \mathbb{R}$ is quasi-continuous and for q.e. $z \in E_{0, T}$,

$$
u(z)=E_{z}\left(\varphi\left(\mathbf{X}_{T_{\iota}}\right)+\int_{0}^{T_{\iota}} f\left(\mathbf{X}_{t}, u\left(\mathbf{X}_{t}\right)\right) d t+\int_{0}^{T_{\iota}} g\left(\mathbf{X}_{t}, u\left(\mathbf{X}_{t}\right)\right) d^{\dagger} \beta_{t}^{\iota}\right) .
$$

Definition. A pair $\left(Y^{z}, M^{z}\right) \in \mathcal{S}^{2} \times \mathcal{M}^{2}$ is a solution of the BDSDE

$$
\begin{aligned}
Y_{t}^{z}= & \varphi\left(\mathbf{X}_{T_{\iota}}\right)+\int_{t}^{T_{\iota}} f\left(\mathbf{X}_{r}, Y_{r}^{z}\right) d r \\
& +\int_{t}^{T_{\iota}} g\left(\mathbf{X}_{r}, Y_{r}^{z}\right) d^{\dagger} \beta_{r}^{\iota}-\int_{t}^{T_{\iota}} d M_{r}^{z}, \quad t \in\left[0, T_{\iota}\right]
\end{aligned}
$$

$\left(\operatorname{BDSDE}_{z}(\varphi, f, g)\right.$ for short) if

(a) $\int_{0}^{T_{\iota}}\left|f\left(\mathbf{X}_{t}, Y_{t}^{z}\right)\right| d t<\infty, \int_{0}^{T_{\iota}}\left\|g\left(\mathbf{X}_{t}, Y_{t}^{z}\right)\right\|^{2} d t<\infty, \mathbb{P}_{z}$-a.s.

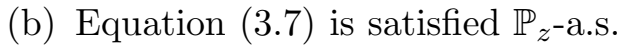

We first prove that under (H1)-(H5) for q.e. $z \in E_{0, T}$ there exists a unique solution of (3.7), and moreover, one can find a version of the solution which is independent of $z$. In fact, the solution exists for every $z \in E_{0, T} \backslash N$, where

$$
N=N_{1} \cup N_{2},
$$

with

$$
\begin{gathered}
N_{1}=\left\{z \in E_{0, T}: \mathbb{E}_{z}\left(\left|\varphi\left(\mathbf{X}_{T_{\iota}}\right)\right|^{2}+\int_{0}^{T_{\iota}}\left(\left|f\left(\mathbf{X}_{t}, 0\right)\right|^{2}+\left\|g\left(\mathbf{X}_{t}, 0\right)\right\|^{2}\right) d t\right)=\infty\right\}, \\
N_{2}=\left\{z \in E_{0, T}: \exists_{y \in \mathbb{R}} \mathbb{P}_{z}\left(\int_{0}^{T_{\iota}}\left|f\left(\mathbf{X}_{r}, y\right)\right| d r=\infty\right)>0\right\} .
\end{gathered}
$$

Lemma 3.2. Let (H1)-(H5) hold and let $N$ be defined by (3.8). Then $\operatorname{cap}(N)=0$ and for every $z \in E_{0, T} \backslash N$ the data

$$
(\xi, \bar{f}(t, y), \bar{g}(t, y))=\left(\varphi\left(\mathbf{X}_{T_{\iota}}\right), f\left(\mathbf{X}_{t}, y\right), g\left(\mathbf{X}_{t}, y\right)\right), \quad t \in[0, T], y \in \mathbb{R}
$$

satisfy assumptions (A1)-(A4) under the measure $\mathbb{P}_{z}$.

Proof. Let $N_{0}=\left\{z \in E_{0, T} ; \mathbb{E}_{z} \int_{0}^{T_{\iota}}\left|f\left(\mathbf{X}_{t}, 0\right)\right|^{2} d t=\infty\right\}$. We may assume that $N_{0}$ is compact. Let $\sigma_{N_{0}}$ be the first hitting time of $N_{0}$. Then

$$
\begin{aligned}
\mathbb{P}_{z}\left(\sigma_{N_{0}}<\zeta_{\iota}\right) & =\mathbb{P}_{z}\left(\exists_{t<\zeta_{\iota}} \mathbf{X}_{t} \in N_{0}\right) \leq \mathbb{P}_{z}\left(\mathbb{E}_{\mathbf{X}_{\sigma_{N_{0}}}} \int_{0}^{T_{\iota}}\left|f\left(\mathbf{X}_{t}, 0\right)\right|^{2} d t=\infty\right) \\
& =\mathbb{P}_{z}\left(\mathbb{E}_{z}\left(\int_{\sigma_{N_{0}}}^{T_{\iota}}\left|f\left(\mathbf{X}_{t}, 0\right)\right|^{2} d t \mid \mathcal{F}_{\sigma_{N_{0}}}^{\mathbf{X}}\right)=\infty\right) \\
& \leq \mathbb{P}_{z}\left(\mathbb{E}_{z}\left(\int_{0}^{T_{\iota}}\left|f\left(\mathbf{X}_{t}, 0\right)\right|^{2} d t \mid \mathcal{F}_{\sigma_{N_{0}}}^{\mathbf{X}}\right)=\infty\right)=0
\end{aligned}
$$


for $m_{T}$-a.e. $z \in E_{0, T}$, because for any strictly positive Borel function $\psi$ on $E_{0, T}$ such that $\left\|\hat{R}^{0, T} \psi\right\|_{\infty}<\infty$ we have

$$
\begin{aligned}
& \int_{E_{0, T}}\left(\mathbb{E}_{z} \int_{0}^{T_{\iota}}\left|f\left(\mathbf{X}_{t}, 0\right)\right|^{2} d t\right) \psi(z) m_{T}(d z)=E\left(R^{0, T} f^{2}(\cdot, 0), \psi\right)_{L^{2}\left(E_{0, T} ; m_{T}\right)} \\
& \quad=E\left(f^{2}(\cdot, 0), \hat{R}^{0, T} \psi\right)_{L^{2}\left(E_{0, T} ; m_{T}\right)} \leq E\|f(\cdot, 0)\|_{L^{2}\left(E_{0, T} ; m_{T}\right)}^{2} \cdot\left\|R^{0, T} \psi\right\|_{\infty}<\infty .
\end{aligned}
$$

Hence $\operatorname{cap}\left(N_{0}\right)=0$. In much the same way one can show that $\operatorname{cap}\left(N_{1}\right)=0$. Moreover, by the definition of the space $q \mathbb{L}^{1}, \operatorname{cap}\left(N_{2}\right)=0$. Thus $\operatorname{cap}(N)=0$ and (A1)-(A4) are satisfied under the measure $\mathbb{P}_{z}$ for $z$ outside the set $N=N_{1} \cup N_{2}$.

In what follows we use the notation

$$
\mathcal{A}_{t}=\mathcal{F}_{T_{\iota}}^{\beta^{\iota}} \vee \mathcal{F}_{t}^{\mathbf{X}}
$$

Theorem 3.3. Assume that $\varphi, f, g$ satisfy (H1)-(H5) and define $N$ by (3.8). Then for every $z \in E_{0, T} \backslash N$ there exists a solution $\left(Y^{z}, M^{z}\right)$ of $\operatorname{BDSDE}_{z}(\varphi, f, g)$. Moreover, there exists a pair of càdlàg $\left(\mathcal{F}_{t}\right)$-adapted processes $(Y, M)$ such that for every $z \in E_{0, T} \backslash N$,

$$
\left(Y_{t}^{z}, M_{t}^{z}\right)=\left(Y_{t}, M_{t}\right), \quad t \in\left[0, T_{\iota}\right], \quad \mathbb{P}_{z^{-} \text {-a.s. }}
$$

Proof. The existence of a solution $\left(Y^{z}, M^{z}\right)$ follows from Theorem 2.6 and Lemma 3.2. The proof of (3.9) we divide into three steps.

Step 1. Assume that $f, g$ do not depend on the last variable $y$. Then

$$
M_{t}^{z}=\mathbb{E}_{z}\left(\varphi\left(\mathbf{X}_{T_{\iota}}\right)+\int_{0}^{T_{\iota}} f\left(\mathbf{X}_{r}\right) d r+\int_{0}^{T_{\iota}} g\left(\mathbf{X}_{r}\right) d^{\dagger} \beta_{r}^{\iota} \mid \mathcal{A}_{t}\right)-Y_{0}^{z}
$$

and

$$
Y_{t}^{z}=\mathbb{E}_{z}\left(\varphi\left(\mathbf{X}_{T_{\iota}}\right)+\int_{t}^{T_{\iota}} f\left(\mathbf{X}_{r}\right) d r+\int_{t}^{T_{\iota}} g\left(\mathbf{X}_{r}\right) d^{\dagger} \beta_{r}^{\iota} \mid \mathcal{A}_{t}\right) .
$$

By [8, Lemma A.3.5] there exists a random variable $H_{0}$ such that $Y_{0}^{z}=H_{0}, \mathbb{P}_{z}$-a.s. for every $z \in E_{0, T} \backslash N$, while by [8, Lemma A.3.3, A.3.5] there exists an $\left(\mathcal{A}_{t}\right)$-adapted càdlàg process $M$ such that $M_{t}=M_{t}^{z}, t \in\left[0, T_{\iota}\right] \mathbb{P}_{z^{-}}$a.s. for $z \in E_{0, T} \backslash N$. Set

$$
Y_{t}=H_{0}-\int_{0}^{t} f\left(\mathbf{X}_{r}\right) d r-\int_{0}^{t} g\left(\mathbf{X}_{r}\right) d^{\dagger} \beta_{r}+\int_{0}^{t} d M_{r}, \quad t \in\left[0, T_{\iota}\right] .
$$

Then $Y$ is an $\left(\mathcal{F}_{t}\right)$-adapted càdlàg process such that $Y_{t}=Y_{t}^{z}, t \in\left[0, T_{\iota}\right], \mathbb{P}_{z}$-a.s. for every $z \in E_{0, T} \backslash N$.

Step 2. We now consider general $f, g$ (possibly depending on $y$ ) but we assume that $f$ is Lipschitz continuous with respect to $y$ uniformly in $t$. By Step 2 of the proof Theorem 2.6 ,

$$
\left(Y^{z, n}, M^{z, n}\right) \rightarrow\left(Y^{z}, M^{z}\right) \text { in } S^{2} \otimes \mathcal{M}^{2},
$$

where $\left(Y^{z, 0}, M^{z, 0}\right)=(0,0)$ and

$$
\begin{aligned}
Y_{t}^{z, n+1}= & \varphi\left(\mathbf{X}_{T_{\iota}}\right)+\int_{t}^{T_{\iota}} f\left(\mathbf{X}_{r}, Y_{r}^{z, n}\right) d r+\int_{t}^{T_{\iota}} g\left(\mathbf{X}_{r}, Y_{r}^{z, n}\right) d^{\dagger} \beta_{r} \\
& -\int_{t}^{T_{\iota}} d M_{r}^{z, n+1}, \quad t \in\left[0, T_{\iota}\right], \quad \mathbb{P}_{z^{-} \text {-a.s. }}
\end{aligned}
$$


for $z \in E_{0, T} \backslash N$. By Step 1, for every $n \geq 0$ there exists a pair $\left(Y^{n}, M^{n}\right)$ of $\left(\mathcal{F}_{t}\right)$-adapted càdlàg processes such that $(3.9)$ holds for $\left(Y^{z}, M^{z}\right),(Y, M)$ replaced by $\left(Y^{z, n}, M^{z, n}\right)$, $\left(Y^{n}, M^{n}\right)$. Therefore applying $[8$, Lemma A.3.3] we show the existence of a pair $(Y, M)$ satisfying (3.9).

Step 3. The general case. From the proof of Theorem 2.6 it follows that (3.11) holds for $\left(Y^{z, n}, M^{z, n}\right)$ being a solution of $\operatorname{BDSDE}_{z}\left(\varphi, f_{n}, g\right)$ with some Lipschitz continuous in $y$ function $f_{n}$. By Step 2, (3.9) holds for $\left(Y^{z, n}, M^{z, n}\right)$. Therefore applying [8, Lemma A.3.3] shows that (3.9) is satisfied.

Of course the pair $(Y, M)$ of Theorem 3.3 is a solution of $\operatorname{BDSDE}_{z}(\varphi, f, g)$ for q.e. $z \in E_{0, T}$. Our next goal is to show that the function $u$ defined as $u(z)=E_{z} Y_{0}$ for $z \in E_{0, T} \backslash N$ is a solution of SPDE (3.4). We first prove this in Proposition 3.6 for linear equations and then in Theorem 3.7 in the general case. We begin with a simple but useful lemma.

Lemma 3.4. Assume that $\Lambda \in \mathcal{A}_{T_{\iota}}$ and let $N$ be a properly exceptional subset of $E_{0, T}$. If $\mathbb{P}_{z}(\Lambda)=1$ for $z \in E_{0, T} \backslash N$ then for every $z \in E_{0, T} \backslash N, \mathbb{P}_{z}\left(\theta_{\tau}^{-1}(\Lambda)\right)=1$ for every stopping time $\tau$ with respect to $\left(\mathcal{A}_{t}\right)$ such that $0 \leq \tau \leq T_{\iota}$.

Proof. By the strong Markov property and proper exceptionality of $N$, for every $z \in$ $E_{0, T} \backslash N$ we have

$$
\mathbb{P}_{z}\left(\theta_{\tau}^{-1}\left(\Lambda^{c}\right)\right)=\mathbb{E}_{z} \mathbb{P}_{\mathbf{X}_{\tau}}\left(\Lambda^{c}\right)=0
$$

which proves the lemma.

Remark 3.5. It is clear that if $\tau$ is an $\left(\mathcal{A}_{t}\right)$-stopping time then $\tau(\omega ; \cdot)$ is an $\left(\mathcal{F}_{t}^{\mathbf{X}}\right)$ stopping time for every $\omega \in \Omega$.

In the sequel we extend the shift operator $\theta$ to $\Omega \times \Omega^{\prime}$ by putting

$$
\theta_{t}\left(\omega, \omega^{\prime}\right)=\left(\omega, \theta_{t}\left(\omega^{\prime}\right)\right)
$$

It is clear that $\theta_{t}^{-1}\left(\mathcal{A}_{0}\right) \subset \mathcal{A}_{t}$.

Proposition 3.6. Assume that $\varphi, f, g$ satisfy (H1), (H2), (H5) and $f, g$ do not depend on the last variable $y$. Define $N$ by (3.8) and set

$$
u(z)=E_{z}\left(\varphi\left(\mathbf{X}_{T_{\iota}}\right)+\int_{0}^{T_{\iota}} f\left(\mathbf{X}_{t}\right) d t+\int_{0}^{T_{\iota}} g\left(\mathbf{X}_{t}\right) d^{\dagger} \beta_{t}^{\iota}\right)
$$

for $z \in E_{0, T} \backslash N$ and $u(z)=0$ otherwise. Then for P-a.s. $\omega \in \Omega$ the function $u(\omega ; \cdot)$ is quasi-continuous and for every $z \in E_{0, T} \backslash N$,

$$
Y_{t}=u\left(\mathbf{X}_{t}\right), \quad t \in\left[0, T_{\iota}\right], \quad \mathbb{P}_{z^{-}} \text {a.s. },
$$

where $Y$ is the process of Theorem 3.3.

Proof. By [8, Theorem 4.1.1] we may and will assume that $N$ is properly exceptional. Let $(Y, M)$ be the pair of processes of Theorem 3.3. We shall show that for every $z \in E_{0, T} \backslash N$,

$$
Y_{0} \circ \theta_{\tau}=Y_{\tau}, \quad \mathbb{P}_{z^{-} \text {-a.s. }}
$$


for every $\left(\mathcal{A}_{t}\right)$-stopping time $\tau$ such that $0 \leq \tau \leq T_{\iota}$. To this end, first observe that $\mathbb{P}_{z}$-a.s. we have

$$
\begin{gathered}
\left(\int_{0}^{T_{\iota}} f\left(\mathbf{X}_{t}\right) d t\right) \circ \theta_{\tau}=\int_{\tau}^{T_{\iota}} f\left(\mathbf{X}_{t}\right) d t \\
\left(\int_{0}^{T_{\iota}} g\left(\mathbf{X}_{t}\right) d^{\dagger} \beta_{t}^{\iota}\right) \circ \theta_{\tau}=\int_{\tau}^{T_{\iota}} g\left(\mathbf{X}_{t}\right) d^{\dagger} \beta_{t}^{\iota}
\end{gathered}
$$

Indeed, the first equation holds since $A_{t}=\int_{0}^{t} f\left(\mathbf{X}_{r}\right) d r$ is a continuous $\mathrm{AF}$ of $\mathbf{M}$ for fixed $\omega \in \Omega$. The second one may be deduced from the identity

$$
\left(\sum_{0 \leq t_{i} \leq T_{\iota}} g\left(\mathbf{X}_{t_{i+1}}\right)\left(\beta_{t_{i+1}}^{\iota}-\beta_{t_{i}}^{\iota}\right)\right) \circ \theta_{\tau}=\sum_{0 \leq t_{i} \leq T_{\iota}-\tau} g\left(\mathbf{X}_{t_{i+1}+\tau}\right)\left(\beta_{t_{i+1}+\tau}^{\iota}-\beta_{t_{\iota}+\tau}^{\iota}\right),
$$

which holds $\mathbb{P}_{z}$-a.s. Here we used the fact that $\iota(0) \circ \theta_{\tau}=\iota(0)+\tau$. It is also clear that

$$
\varphi\left(\mathbf{X}_{T_{\iota}}\right) \circ \theta_{\tau}=\varphi\left(\mathbf{X}_{T_{\iota}}\right) .
$$

By [26, Theorem 50.19], $\left(N_{t}=\left(M_{t-\tau} \circ \theta_{\tau}-M_{0} \circ \theta_{\tau}\right) \mathbf{1}_{[\tau, \infty)}(t), \mathcal{F}_{t}^{\mathbf{X}}, t \geq 0\right)$ is a martingale for fixed $\omega \in \Omega$. Hence

$$
\mathbb{E}_{z}\left(\left(\int_{0}^{T_{\iota}} d M_{t}\right) \circ \theta_{\tau} \mid \mathcal{A}_{\tau}\right)=E_{z}\left(\left(\int_{0}^{T_{\iota}} d M_{t}\right) \circ \theta_{\tau} \mid \mathcal{F}_{\tau}^{\mathbf{X}}\right)=E_{z}\left(N_{T_{\iota}+\tau} \mid \mathcal{F}_{\tau}^{\mathbf{X}}\right)=0
$$

We know that for every $z \in E_{0, T} \backslash N$,

$$
Y_{0}=\varphi\left(\mathbf{X}_{T_{\iota}}\right)+\int_{0}^{T_{\iota}} f\left(\mathbf{X}_{r}\right) d r-\int_{0}^{T_{\iota}} g\left(\mathbf{X}_{r}\right) d^{\dagger} \beta_{r}^{\iota}-\int_{0}^{T_{\iota}} d M_{r}, \quad \mathbb{P}_{z^{-}} \text {-a.s. }
$$

By Lemma 3.4 and (3.15)-(3.17), for every $z \in E_{0, T} \backslash N$,

$$
Y_{0} \circ \theta_{\tau}=\varphi\left(\mathbf{X}_{T_{\iota}}\right)+\int_{\tau}^{T_{\iota}} f\left(\mathbf{X}_{r}\right) d r-\int_{\tau}^{T_{\iota}} g\left(\mathbf{X}_{r}\right) d^{\dagger} \beta_{r}^{\iota}-\left(\int_{0}^{T_{\iota}} d M_{r}\right) \circ \theta_{\tau}, \quad \mathbb{P}_{z} \text {-a.s. }
$$

Since $Y_{0}$ is $\mathcal{A}_{0}$-measurable, $Y_{0} \circ \theta_{\tau}$ is $\mathcal{A}_{\tau}$-measurable. Therefore by (3.18),

$$
Y_{0} \circ \theta_{\tau}=\mathbb{E}_{z}\left(\varphi\left(\mathbf{X}_{T_{\iota}}\right)+\int_{\tau}^{T_{\iota}} f\left(\mathbf{X}_{r}\right) d r-\int_{\tau}^{T_{\iota}} g\left(\mathbf{X}_{r}\right) d^{\dagger} \beta_{r}^{\iota} \mid \mathcal{A}_{\tau}\right), \quad \mathbb{P}_{z^{-}} \text {a.s. }
$$

Thus (3.14) is satisfied for $z \in E_{0, T} \backslash N$. Since $u(z)=E_{z} Y_{0}$, using the strong Markov property and (3.14) we see that for every $z \in E_{0, T} \backslash N$,

$$
u\left(\mathbf{X}_{\tau}\right)=E_{\mathbf{X}_{\tau}} Y_{0}=E_{z}\left(Y_{0} \circ \theta_{\tau} \mid \mathcal{F}_{\tau}^{X}\right)=E_{z}\left(Y_{\tau} \mid \mathcal{F}_{\tau}^{X}\right)=\mathbb{E}_{z}\left(Y_{\tau} \mid \mathcal{A}_{\tau}\right)=Y_{\tau}, \quad \mathbb{P}_{z} \text {-a.s. }
$$

A standard approximation argument shows that the process $u(\mathbf{X})$ is optional (with respect to $\left.\left(\mathcal{A}_{t}\right)\right)$. Therefore the above equality implies (3.13) by the Section Theorem. To prove quasi-continuity of $u(\omega ; \cdot)$ for $P$-a.s. $\omega \in \Omega$, fix $\omega \in \Omega$ such that (3.13) holds $P_{m_{T}}$-a.s. Let $\tau$ be a predictable $\left(\mathcal{F}_{t}^{\mathbf{X}}\right)$-stopping time such that $0 \leq \tau \leq T_{\iota}$. Since $\mathbf{X}$ is a Hunt process, $X_{\tau-}=X_{\tau}, P_{z^{-}}$-a.s. for every $z \in E_{0, T}$, and moreover, the filtration $\left(\mathcal{F}_{t}^{\mathbf{X}}\right)$ 
is quasi-left continuous, so $M_{\tau-}=M_{\tau}, P_{z}$-a.s. for every $z \in E_{0, T}$ (see [8, Theorems A.3.2, A.3.6]). By this and (3.13),

$$
u\left(\mathbf{X}_{\tau-}\right)=u\left(\mathbf{X}_{\tau}\right)=u(\mathbf{X})_{\tau-}, \quad P_{m_{T}} \text {-a.s. }
$$

Of course the process $\left\{u(\mathbf{X})_{t-}, t \geq 0\right\}$ is predictable. Since the function $u(\omega ; \cdot)$ is nearly Borel, the process $\left\{u\left(\mathbf{X}_{t-}\right), t \geq 0\right\}$ is predictable, too. Therefore applying the Section Theorem yields

$$
u\left(\mathbf{X}_{t-}\right)=u(\mathbf{X})_{t-}, \quad t \in\left[0, T_{\iota}\right], \quad P_{m_{T}} \text {-a.s. },
$$

which together with $(3.13)$ and [16, Theorem IV.5.29] shows that $u(\omega ; \cdot)$ is quasicontinuous.

Theorem 3.7. Assume that $\varphi, f, g$ satisfy (H1)-(H5). Let $N$ be defined by (3.8) and let $Y$ be the process of Theorem 3.3. Then for P-a.s. $\omega \in \Omega$ the function $u(\omega ; \cdot)$ : $E_{0, T} \backslash N \rightarrow \mathbb{R}$ defined as

$$
u(z)=E_{z}\left(\varphi\left(\mathbf{X}_{T_{\iota}}\right)+\int_{0}^{T_{\iota}} f\left(\mathbf{X}_{t}, Y_{t}\right) d t+\int_{0}^{T_{\iota}} g\left(\mathbf{X}_{t}, Y_{t}\right) d^{\dagger} \beta_{t}^{\iota}\right)
$$

is quasi-continuous and for every $z \in E_{0, T} \backslash N$,

$$
Y_{t}=u\left(\mathbf{X}_{t}\right), \quad t \in\left[0, T_{\iota}\right], \quad \mathbb{P}_{z^{-}} \text {a.s. }
$$

In particular, $u$ is a solution of (3.4).

Proof. By [8, Theorem 4.1.1] we may and will assume that $N$ is properly exceptional. Let $(Y, M)$ be the pair of processes of Theorem 3.3. By the proof of Theorem 2.6, for every $z \in E_{0, T} \backslash N$ the pair $(Y, M)$ is under $\mathbb{P}_{z}$ a limit in $S^{2} \otimes \mathcal{M}^{2}$ of solutions $\left(Y^{n}, M^{n}\right)$ of some linear BDSDEs. In particular, $u_{n}(z)=E_{z} Y_{0}^{n} \rightarrow E_{z} Y_{0}=u(z)$ for every $z \in E_{0, T} \backslash N$, which when combined with the fact that $N$ is properly exceptional implies that $u_{n}\left(\mathbf{X}_{t}\right) \rightarrow u\left(\mathbf{X}_{t}\right), t \in\left[0, T_{\iota}\right], \mathbb{P}_{z}$-a.s. for every $z \in E_{0, T} \backslash N$. Since we know from Proposition 3.6 that (3.20) holds for solutions of linear equations, we conclude that (3.20) holds in the general case. Quasi-continuity of $u$ follows now from Proposition 3.6. Since by (3.19), (3.20) and Theorem 3.3 conditions (a) (b) of the definition of a solution SPDE (3.4) are satisfied, $u$ is a solution of (3.4).

Remark 3.8. Assume (H1)-(H5).

(i) From Theorems 3.3 and 3.7 we know that there exists a pair $(Y, M)$ (not depending on $z)$ of $\left(\mathcal{F}_{t}\right)$-adapted càdlàg processes such that $(Y, M)$ is a solution of $\operatorname{BDSDE}_{z}(\varphi, f, g)$ for q.e. $z \in E_{0, T}$ and that the solution $(Y, M)$ provides a stochastic representation of the solution $u$ of SPDE (3.4). The representation has the form (3.19), or equivalently,

$$
u(z)=Y_{0}, \quad \mathbb{P}_{z} \text {-a.s. for q.e. } z \in E_{0, T} \text {. }
$$

(ii) On the contrary, if $u$ is a solution of (3.4) then there is an $\left(\mathcal{F}_{t}\right)$-adapted càdlàg process $M$ (not depending on $z$ ) such that for q.e. $z \in E_{0, T}$ the pair $(u(\mathbf{X}), M)$ is a solution of $\operatorname{BDSDE}_{z}(\varphi, f, g)$. Indeed, given a solution $u$ let us define $f_{u}, g_{u}$ as $f_{u}(z)=f(z, u(z)), g_{u}(z)=g(z, u(z)), z \in E_{0, T}$. Let $(Y, M)$ be the pair of processes of Theorem 3.3 such that $(Y, M)$ is a solution of the linear $\operatorname{BDSDE}_{z}\left(\varphi, f_{u}, g_{u}\right)$ for 
q.e. $z \in E_{0, T}$. By Proposition 3.6, $Y=u(\mathbf{X})$, so the pair $(u(\mathbf{X}), M)$ is a solution of $\operatorname{BDSDE}_{z}\left(\varphi, f_{u}, g_{u}\right)$, which means that it is a solution of $\operatorname{BDSDE}_{z}(\varphi, f, g)$. Thus starting from $u$ we can construct a solution $(u(\mathbf{X}), M)$ of the BDSDE

$$
\begin{aligned}
u\left(\mathbf{X}_{t}\right)= & \varphi\left(\mathbf{X}_{T_{\iota}}\right)+\int_{t}^{T_{\iota}} f\left(\mathbf{X}_{r}, u\left(\mathbf{X}_{r}\right)\right) d r \\
& +\int_{0}^{T_{\iota}} g\left(\mathbf{X}_{r}, u\left(\mathbf{X}_{r}\right)\right) d^{\dagger} \beta_{r}^{\iota}-\int_{t}^{T_{\iota}} d M_{r}, \quad t \in\left[0, T_{\iota}\right], \quad \mathbb{P}_{z^{-} \text {-a.s. }}
\end{aligned}
$$

Proposition 3.9. Under (H1)-(H5) there exists at most one solution of (3.4).

Proof. Let $u_{1}, u_{2}$ be two solutions of (3.4). By Remark 3.8, for q.e. $z \in E_{0, T}$ the processes $u_{1}(\mathbf{X}), u_{2}(\mathbf{X})$ are the first components of solutions of $\operatorname{BDSDE}_{z}(\varphi, f, g)$. Therefore by Corollary 2.4 and Lemma 3.2, for q.e. $z \in E_{0, T}$ we have $u_{1}(z)=u_{2}(z) P$-a.s.

In the next section we prove some results on regularity of the solution $u$ of SPDE (3.4). Here let us only note that our proofs are based on equation (3.21). Clearly (3.21) implies that for q.e. $z \in E_{0, T}$,

$$
A_{t}:=u\left(\mathbf{X}_{t}\right)-u\left(\mathbf{X}_{0}\right)=M_{t}+N_{t}, \quad t \in\left[0, T_{\iota}\right], \quad \mathbb{P}_{z} \text {-a.s. }
$$

with $N$ defined by (1.8). We shall see that $M$ is a random martingale AF of finite energy and $N$ is a random continuous AF of finite, but in most cases nonzero energy. This means that (3.22) can not be viewed as Fukushima's decomposition of $A$. Nevertheless, we will prove some estimates for the energy $e(M)$ of $M$, which when combined with a priori estimates for $(u(\mathbf{X}), M)$ obtained in Proposition 2.5 lead to energy estimates for $u$.

\section{Regularity of solutions}

Let $\mathcal{H}$ be some Hilbert space equipped with the norm $|\cdot|_{\mathcal{H}}$, let $\mathcal{B}(\mathcal{H})$ denote the Borel $\sigma$-field of subsets of $\mathcal{H}$ and let

- $M^{2}(0, T ; \mathcal{H})$ be the space of all $\mathcal{F} \otimes \mathcal{B}([0, T]) / \mathcal{B}(\mathcal{H})$ measurable processes $v$ : $\Omega \times[0, T] \rightarrow \mathcal{H}$ such that for a.e. $t \in[0, T]$ the random variable $v(t)$ is $\mathcal{F}_{t, T^{-}}^{\beta}$ measurable and

$$
E \int_{0}^{T}|v(t)|_{\mathcal{H}}^{2} d t<\infty
$$

- $S^{2}(0, T ; \mathcal{H})$ be the subspace of $M^{2}(0, T ; \mathcal{H})$ consisting of all processes $v$ such that

$$
\sup _{0 \leq t \leq T} E|v(t)|_{\mathcal{H}}^{2}<\infty
$$

Let $u$ be a solution of (3.4) of Theorem 3.7 and let $u(t)=u(t, \cdot)$. In this section we show that $t \mapsto u(t)$ belongs to the space $S^{2}(0, T ; H) \cap M^{2}(0, T ; V)$ with $H, V$ defined in Section 3.1, and we prove energy estimates for $u$, i.e. estimates of $u$ in the norm $\|\cdot\|_{\mathcal{B}^{0, T}}$ defined as $\|u\|_{\mathcal{B}^{0, T}}^{2}=\mathcal{B}^{0, T}(u, u)$, where $\mathcal{B}^{0, T}(u, u)$ is defined by (3.2). Note that by assumption (b) in Section 3.1, the norm $\|\cdot\|_{\mathcal{B}^{0, T}}$ is equivalent to the usual norm in the space $L^{2}(0, T ; V)$. We begin with linear equations. 
Proposition 4.1. Assume that $f, g$ do not depend on the last variable $y$ and (H1) is satisfied. Then $u$ defined by (3.12) belongs to $M^{2}(0, T ; V)$ and there is $c>0$ such that

$$
E\|u\|_{\mathcal{B}^{0, T}}^{2} \leq c E\left(\|\varphi\|_{L^{2}(E ; m)}^{2}+\|f\|_{L^{2}\left(E_{0, T} ; m_{T}\right)}^{2}+\sum_{k=1}^{\infty}\left\|g_{k}\right\|_{L^{2}\left(E_{0, T} ; m_{T}\right)}^{2}\right) .
$$

Proof. Put

$$
w(z)=E_{z}\left(\varphi\left(\mathbf{X}_{T_{\iota}}\right)+\int_{0}^{T_{\iota}} f\left(\mathbf{X}_{t}\right) d t\right), \quad z \in E_{0, T} .
$$

From [12, Theorem 3.7] and standard energy estimates for solutions of PDEs it follows that $w \in M^{2}(0, T ; V)$ and

$$
\|w\|_{\mathcal{B}^{0, T}}^{2} \leq\|\varphi\|_{L^{2}(E ; m)}^{2}+\|f\|_{L^{2}\left(E_{0, T} ; m_{T}\right)}^{2} .
$$

Put

$$
v(z)=E_{z} \int_{0}^{T_{\iota}} g\left(\mathbf{X}_{t}\right) d^{\dagger} \beta_{t}^{\iota}, \quad z \in E_{0, T}
$$

We are going to show that $v \in M^{2}(0, T ; V)$ and

$$
E\|v\|_{\mathcal{B}^{0, T}}^{2} \leq c \sum_{k \geq 1} E\left\|g_{k}\right\|_{L^{2}\left(E_{0, T} ; m_{T}\right)}^{2} .
$$

To this end, let us first observe that by the stochastic Fubini theorem and Markov property,

$$
\begin{aligned}
\left(R_{\alpha}^{0, T} v\right)(z) & =E_{z} \int_{0}^{T_{\iota}} e^{-\alpha r} E_{\mathbf{X}_{r}}\left(\int_{0}^{T_{\iota}} g\left(\mathbf{X}_{t}\right) d^{\dagger} \beta_{t}^{\iota}\right) d r \\
& =E_{z} \int_{0}^{T_{\iota}} e^{-\alpha r} E_{z}\left(\int_{r}^{T_{\iota}} g\left(\mathbf{X}_{t}\right) d^{\dagger} \beta_{t}^{\iota} \mid \mathcal{F}_{r}^{\mathbf{X}}\right) d r \\
& =E_{z} \int_{0}^{T_{\iota}} e^{-\alpha r}\left(\int_{r}^{T_{\iota}} g\left(\mathbf{X}_{t}\right) d^{\dagger} \beta_{t}^{\iota}\right) d r=\frac{1}{\alpha} E_{z} \int_{0}^{T_{\iota}}\left(1-e^{-\alpha t}\right) g\left(\mathbf{X}_{t}\right) d^{\dagger} \beta_{t}^{\iota} .
\end{aligned}
$$

Hence

$$
v(z)-\alpha\left(R_{\alpha}^{0, T} v\right)(z)=E_{z} \int_{0}^{T_{\iota}} e^{-\alpha t} g\left(\mathbf{X}_{t}\right) d^{\dagger} \beta_{t}^{\iota}, \quad z \in E_{0, T} .
$$

For given $\alpha>0$ and $v, u \in M^{2}(0, T ; V)$ write

$$
\mathcal{E}^{(\alpha), 0, T}(u, v)=\alpha\left(u-\alpha R_{\alpha}^{0, T} u, v\right)_{L^{2}\left(E_{0, T} ; m_{T}\right)} .
$$

By (4.2),

$$
E \mathcal{E}^{(\alpha), 0, T}(v, v)=\alpha \int_{E_{0, T}} E\left(E_{z} \int_{0}^{T_{\iota}} e^{-\alpha t} g\left(\mathbf{X}_{t}\right) d^{\dagger} \beta_{t}^{\iota} \cdot E_{z} \int_{0}^{T_{\iota}} g\left(\mathbf{X}_{t}\right) d^{\dagger} \beta_{t}^{\iota}\right) m_{T}(d z) .
$$

Using Itô's isometry and the fact that $\alpha \hat{R}_{\alpha}^{0, T}$ is a contraction on $L^{2}\left(E_{0, T} ; m_{T}\right)$ we conclude from the above that

$$
\begin{aligned}
E \mathcal{E}^{(\alpha), 0, T}(v, v) & \leq \int_{E_{0, T}} E\left(E_{z} \int_{0}^{T_{\iota}} e^{-\alpha t}\left|g\left(\mathbf{X}_{t}\right)\right|^{2} d t\right) m_{T}(d z) \\
& =\sum_{k=1}^{\infty} E\left\|\alpha R_{\alpha}^{0, T} g_{k}^{2}\right\|_{L^{1}\left(E_{0, T} ; m_{T}\right)} \leq \sum_{k=1}^{\infty} E\left\|g_{k}\right\|_{L^{2}\left(E_{0, T} ; m_{T}\right)}^{2} .
\end{aligned}
$$


By $[19,(6.1 .28)]$,

$$
E \mathcal{B}^{0, T}\left(\alpha R_{\alpha}^{0, T} v, \alpha R_{\alpha}^{0, T} v\right) \leq E \mathcal{E}^{(\alpha), 0, T}(v, v) .
$$

When combined with (4.3) and [16, Theorem I.2.13] this shows that $v \in M^{2}(0, T ; V)$. Therefore letting $\alpha \rightarrow \infty$ in (4.3) yields (4.1).

Lemma 4.2. Let $u: \Omega \times E_{0, T} \rightarrow \mathbb{R}_{+}$and let $u_{\alpha}=\alpha R_{\alpha}^{0, T} u, \alpha>0$.

(i) If $u \in M^{2}(0, T ; H)$ then $E\left\|u_{\alpha}-u\right\|_{L^{2}\left(E_{0, T} ; m_{T}\right)}^{2} \rightarrow 0$ as $\alpha \rightarrow \infty$.

(ii) If $u \in M^{2}(0, T ; V)$ then $E \mathcal{B}^{0, T}\left(u_{\alpha}-u, u_{\alpha}-u\right) \rightarrow 0$ as $\alpha \rightarrow \infty$.

(iii) If $u(z)=E_{z} \int_{0}^{T_{\iota}} g\left(\mathbf{X}_{t}\right) d^{\dagger} \beta_{t}$ for some $g$ satisfying $(\mathrm{H} 1)$ and not depending on $y$, then for every $t \in[0, T), E\left\|u_{\alpha}(t)-u(t)\right\|_{L^{2}(E ; m)}^{2} \rightarrow 0$ as $\alpha \rightarrow \infty$.

Proof. (i) Since $\left\{R_{\alpha}^{0, T}\right\}$ is a strongly continuous contraction resolvent on $L^{2}\left(E_{0, T} ; m_{T}\right)$, $u_{\alpha} \rightarrow u$ in $L^{2}\left(E_{0, T} ; m_{T}\right)$ and $\left\|u_{\alpha}\right\|_{L^{2}\left(E_{0, T} ; m_{T}\right)} \leq\|u\|_{L^{2}\left(E_{0, T} ; m_{T}\right)}$ for $P$-a.e. $\omega \in \Omega$. Therefore applying the Lebesgue dominated convergence theorem we get (i).

(ii) By [16, Theorem I.2.13], $\mathcal{B}^{0, T}\left(u_{\alpha}-u, u_{\alpha}-u\right) \rightarrow 0$ for $P$-a.e. $\omega \in \Omega$. Moreover, by [16, Lemma I.2.11], there exists $c>0$ (independent of $\omega$ ) such that $\mathcal{B}^{0, T}\left(u_{\alpha}, u_{\alpha}\right) \leq$ $c \mathcal{B}^{0, T}(u, u)$ for $P$-a.e. $\omega \in \Omega$. Therefore (ii) follows by the Lebesgue dominated theorem. (iii) By (4.2),

$$
E\left\|u_{\alpha}(t)-u(t)\right\|_{L^{2}(E ; m)}^{2} \leq \mathbb{E}_{t, m} \int_{0}^{T_{\iota}} e^{-2 \alpha r}\left\|g\left(\mathbf{X}_{r}\right)\right\|^{2} d r
$$

so it suffices to show that the integral on the right-hand side of the above inequality is finite for every $t \in[0, T)$. But

$$
\mathbb{E}_{t, m} \int_{0}^{T_{\iota}}\left\|g\left(\mathbf{X}_{r}\right)\right\|^{2} d r=E E_{t, m} \int_{t}^{T} \sum_{k=1}^{\infty}\left|g_{k}\left(r, X_{r}\right)\right|^{2} d r \leq E\|g\|_{L^{2}\left(E_{0, T} ; m_{T}\right)}^{2},
$$

which implies the desired conclusion.

Let us recall that the energy $e(A)$ of an $\mathrm{AF} A$ of the process $\mathbf{M}^{0, T}$ associated with the form $\mathcal{E}^{0, T}$ is defined as

$$
e(A)=\frac{1}{2} \lim _{\alpha \rightarrow \infty} \alpha^{2} E_{m_{T}} \int_{0}^{T_{\iota}} e^{-\alpha t} A_{t}^{2} d t
$$

whenever the integral exists (see, e.g., $[17,19]$ ). Also note that if $M$ is a martingale $\mathrm{AF}$ of $\mathbf{M}^{0, T}$ then the sharp bracket $\langle M\rangle$ of $M$ is a positive continuous $\mathrm{AF}$ of $\mathbf{M}^{0, T}$. Let $\mu_{\langle M\rangle}$ denote the Revuz measure of $\langle M\rangle$. Then

$$
\mu_{\langle M\rangle}\left(E_{0, T}\right)=\lim _{t \rightarrow 0^{+}} \frac{1}{t} E_{m_{T}}\langle M\rangle_{t}=2 e(M)
$$

(see [19, Section 5.1.2]).

In what follows we will be interested in AFs of the form

$$
A_{t}=\tilde{u}\left(\mathbf{X}_{t}\right)-\tilde{u}\left(\mathbf{X}_{0}\right), \quad t \in\left[0, T_{\iota}\right]
$$


where $\tilde{u}$ is a quasi-continuous $m_{T}$-version of $u \in \mathcal{W}_{T}$. Such AFs admit a unique decomposition (called Fukushima's decomposition)

$$
A_{t}=M_{t}^{[u]}+N_{t}^{[u]}, \quad t \in\left[0, T_{\iota}\right]
$$

into a martingale AF $M^{[u]}$ of $\mathbf{M}^{0, T}$ of finite energy and a continuous AF $N^{[u]}$ of $\mathbf{M}^{0, T}$ of zero energy (see [17, Theorem 6.4]).

Lemma 4.3. Let $k^{\beta}=\beta\left(1-\beta \hat{R}_{\beta}^{0, T} 1\right) \cdot m_{T}$. Then there exists a smooth Radon measure $k$ such that $k^{\beta} \rightarrow k$ in the vague topology as $\beta \rightarrow \infty$, and for every $u \in \mathcal{W}_{T}$,

$$
e\left(M^{[u]}\right)=\|\tilde{u}(0)\|_{L^{2}(E ; m)}^{2}+\mathcal{B}^{0, T}(u, u)-\frac{1}{2} \int_{E_{0, T}}|\tilde{u}(z)|^{2} k(d z),
$$

where $\tilde{u}$ is a quasi-continuous $m_{T}$-version of $u$.

Proof. Let $A$ be defined by (4.5). By an elementary computation we get

$$
\beta^{2} E_{m_{T}} \int_{0}^{T_{\iota}} e^{-\beta t}\left(u\left(\mathbf{X}_{t}\right)-u\left(\mathbf{X}_{0}\right)\right)^{2} d t=2 \beta\left(u, u-\beta R_{\beta}^{0, T} u\right)-\beta\left(u^{2}, 1-\beta \hat{R}_{\beta}^{0, T} 1\right) .
$$

Hence

$$
\beta^{2} E_{m_{T}} \int_{0}^{T_{\iota}} e^{-\beta t} A_{t}^{2} d t=2 \mathcal{E}^{(\beta), 0, T}(u, u)-\left(u^{2}, k^{\beta}\right)
$$

By (4.7),

$$
\left(u^{2}, k^{\beta}\right) \leq 2 \mathcal{E}^{(\beta), 0, T}(u, u)
$$

From this we conclude that the sequence $\left\{k^{\beta}\right\}$ is tight in the vague topology. Therefore if $u \in \mathcal{W}_{T} \cap C_{c}\left(E_{T}\right)$ then letting $\beta \rightarrow \infty$ in the above inequality we get

$$
\left(u^{2}, k\right) \leq 2 \mathcal{E}^{0, T}(u, u)=\|u(0)\|_{L^{2}(E ; m)}^{2}+2 \mathcal{B}^{0, T}(u, u)
$$

Since it is known that there is a continuous embedding of $\mathcal{W}_{T}$ into $C([0, T] ; H)$, from (4.8) it follows that there is $c>0$ such that

$$
\left(u^{2}, k\right) \leq c\|u\|_{\mathcal{W}_{T}}
$$

for all $u \in \mathcal{W}_{T} \cap C_{c}\left(E_{T}\right)$. From (4.9) and [23, Theorem 1] it follows that $k$ is a smooth measure. Furthermore, since each quasi-continuous $u \in \mathcal{W}_{T}$ can be approximated q.e. and in $\mathcal{W}_{T}$ by functions from the space $\mathcal{W}_{T} \cap C_{c}\left(E_{T}\right)$, (4.8) holds true for every quasicontinuous $u \in \mathcal{W}_{T}$. Let $\left\{u_{n}\right\} \subset \mathcal{W}_{T} \cap C_{c}\left(E_{T}\right)$ be such that $u_{n} \rightarrow u$ in $\mathcal{W}_{T}$. Then

$$
\begin{aligned}
\left|\left(u^{2}, k^{\beta}\right)^{1 / 2}-\left(u^{2}, k\right)^{1 / 2}\right| \leq & \left|\left(u_{n}^{2}, k^{\beta}\right)^{1 / 2}-\left(u_{n}^{2}, k\right)^{1 / 2}\right|+\left\|\left(u_{n}-u\right)(0)\right\|_{L^{2}} \\
& +\left(2 \mathcal{E}^{(\beta), 0, T}\left(u_{n}-u, u_{n}-u\right)\right)^{1 / 2} \\
& +\left(2 \mathcal{B}^{0, T}\left(u_{n}-u, u_{n}-u\right)\right)^{1 / 2}
\end{aligned}
$$

Since $\mathcal{W}_{T} \subset C\left(0, T ; L^{2}(E ; m)\right)$ and the embedding is continuous, letting $\beta \rightarrow \infty$ and then $n \rightarrow \infty$ in (4.10) shows that for every $u \in \mathcal{W}_{T}$,

$$
\int_{E_{0, T}} u^{2} d k^{\beta} \rightarrow \int_{E_{0, T}} u^{2} d k
$$

as $\beta \rightarrow \infty$. By [17, Theorem 6.4], $e(A)=e\left(M^{[u]}\right)$. Therefore letting $\beta \rightarrow \infty$ in (4.7) yields $2 e\left(M^{[u]}\right)=2 \mathcal{E}^{0, T}(u, u)-\left(u^{2}, k\right)$, which implies $(4.6)$. 
Definition. We say that an $\left(\mathcal{F}_{t}\right)$-adapted process $A$ is a random additive functional (random AF for short) of $\mathbf{M}^{0, T}$ if for $P$-a.e. $\omega \in \Omega$ the process $A(\omega ; \cdot)$ is an $\mathrm{AF}$ of $\mathbf{M}^{0, T}$. Similarly, we say that a process $A$ is a random martingale (continuous, positive) AF of $\mathbf{M}^{0, T}$ if for $P$-a.e. $\omega \in \Omega$ the process $A(\omega ; \cdot)$ is a martingale (continuous, positive) AF of $\mathbf{M}^{0, T}$.

Lemma 4.4. Let $\left\{A^{n}\right\}$ be a sequence of random AFs such that

$$
\mathbb{E}_{z} \sup _{t \leq T_{\iota}}\left|A_{t}^{n}-A_{t}^{m}\right| \rightarrow 0 \quad \text { for } m_{T} \text {-a.e. } z \in E_{0, T} \text { as } n, m \rightarrow \infty \text {. }
$$

Then there exists a subsequence $\left(n_{k}\right) \subset(n)$ such that for $P$-a.s. $\omega \in \Omega$,

$$
E_{z} \sup _{t \leq T_{\iota}}\left|A_{t}^{n_{k}}-A_{t}^{n_{l}}\right| \rightarrow 0 \quad \text { for q.e. } z \in E_{0, T} \text { as } k, l \rightarrow \infty .
$$

Proof. Let $\rho \in L^{1}\left(E_{0, T} ; m_{T}\right)$ be a strictly positive function such that $\int_{E_{0, T}} \rho d m_{T}=1$ and

$$
\mathbb{E}_{\nu} \sup _{t \leq T_{\iota}}\left|A_{t}^{n}-A_{t}^{m}\right| \rightarrow 0 \quad \text { as } n, m \rightarrow \infty
$$

where $\nu=\rho \cdot m_{T}$. Let $\left(n_{k}\right) \subset(n)$ be a subsequence such that

$$
\mathbb{E}_{\nu} \sup _{t \leq T_{\iota}}\left|A_{t}^{n_{k+1}}-A_{t}^{n_{k}}\right| \leq 2^{-k}, \quad k \geq 1
$$

and let $B=\left\{z \in E_{0, T}: E_{z} \sup _{t \leq T_{\iota}}\left|A_{t}^{n_{k}}-A_{t}^{n_{l}}\right| \nrightarrow 0\right.$ as $\left.k, l \rightarrow \infty\right\}$. Let us stress that $B$ depends on $\omega$. Write $\tau=\sigma_{B}$. By the Markov property and additivity of $A$,

$$
\begin{aligned}
P_{\nu}\left(\tau<T_{\iota}\right) & \leq \int_{E_{0, T}} P_{z}\left(\exists_{s \leq T_{\iota}} E_{z}\left(\sup _{t \leq T_{\iota} \circ \theta_{s}} \mid A_{t}^{n_{l}} \circ \theta_{s}-A_{t}^{n_{k}} \circ \theta_{s} \| \mathcal{F}_{s}^{\mathbf{X}}\right) \nrightarrow 0\right) d \nu(z) \\
& =\int_{E_{0, T}} P_{z}\left(\exists_{s \leq T_{\iota}} E_{z}\left(\sup _{t+s \leq T_{\iota}}\left|A_{t+s}^{n_{l}}-A_{s}^{n_{l}}-A_{t+s}^{n_{k}}+A_{s}^{n_{k}}\right| \mid \mathcal{F}_{s}^{\mathbf{X}}\right) \nrightarrow 0\right) d \nu(z) \\
& \leq \int_{E_{0, T}} P_{z}\left(\exists_{s \leq T_{\iota}} 2 E_{z}\left(\sup _{t \leq T_{\iota}} \mid A_{t}^{n_{l}}-A_{t}^{n_{k}} \| \mathcal{F}_{s}^{\mathbf{X}}\right) \nrightarrow 0\right) d \nu(z) \\
& \leq \int_{E_{0, T}} P_{z}\left(\sup _{0 \leq s \leq T_{\iota}}\left(E_{z}\left(\sup _{t \leq T_{\iota}} \mid A_{t}^{n_{l}}-A_{t}^{n_{k}} \| \mathcal{F}_{s}^{\mathbf{X}}\right)\right)^{q} \nrightarrow 0\right) d \nu(z)
\end{aligned}
$$

for every $q \in(0,1)$. Let $\Pi=P \otimes(\nu \otimes K)$, where $K\left(z, d \omega^{\prime}\right)=P_{z}\left(d \omega^{\prime}\right)$. By $(4.11)$ and [4, Lemma 6.1],

$$
\begin{aligned}
& \sum_{k=1}^{\infty} \int_{\Omega \times E_{0, T} \times \Omega^{\prime}} \sup _{s \leq T_{\iota}}\left(E_{z}\left(\sup _{t \leq T_{\iota}}\left|A_{t}^{n_{k+1}}-A_{t}^{n_{k}}\right| \mid \mathcal{F}_{s}^{\mathbf{X}}\right)\right)^{q} d \Pi\left(\omega, z, \omega^{\prime}\right) \\
& \quad \leq \sum_{k=1}^{\infty} \frac{1}{1-q}\left(\mathbb{E}_{\nu} \sup _{t \leq T_{\iota}}\left|A_{t}^{n_{k+1}}-A_{t}^{n_{k}}\right|\right)^{q}<\infty
\end{aligned}
$$

Since $\Pi\left(\Omega \times E_{0, T} \times \Omega^{\prime}\right)=1$, applying the Borel-Cantelli lemma shows that

$$
\sup _{s \leq T_{\iota}}\left(E .\left(\sup _{t \leq T_{\iota}} \mid A_{t}^{n_{k}}-A_{t}^{n_{l}} \| \mathcal{F}_{s}^{\mathbf{X}}\right)\right)^{q} \rightarrow 0 \quad \text { as } k, l \rightarrow \infty, \quad \text { I-a.e. }
$$


In particular, for $\nu$-a.e. $z \in E_{0, T}$ and $P$-a.e. $\omega \in \Omega$,

$$
\sup _{s \leq T_{\iota}}\left(E_{z}\left(\sup _{t \leq T_{\iota}} \mid A_{t}^{n_{k}}-A_{t}^{n_{l}} \| \mathcal{F}_{s}^{\mathbf{X}}\right)\right)^{q} \rightarrow 0 \quad \text { as } k, l \rightarrow \infty, \quad P_{z^{-} \text {-a.e. }}
$$

Hence $\mathbb{P}_{\nu}\left(\tau \leq T_{\iota}\right)=0$, which implies that $\operatorname{cap}(B)=0$-a.e. (see $[18$, p. 298$\left.]\right)$.

For a given set $A \subset E_{0, T} \times \Omega$ and $\left(z_{0}, \omega_{0}\right)$ we write $A_{z_{0}}=\left\{\omega \in \Omega ;\left(z_{0}, \omega\right) \in A\right\}$ and $A_{\omega_{0}}=\left\{z \in E_{0, T} ;\left(z, \omega_{0}\right) \in A\right\}$.

Lemma 4.5. Let $A \subset E_{0, T} \times \Omega$ be a measurable set. If $\operatorname{cap}\left(A_{\omega}\right)=0$ for $P$-a.e. $\omega \in \Omega$, then $P\left(A_{z}\right)=0$ for cap-q.e. $z \in E_{0, T}$.

Proof. Let $\nu$ be a smooth bounded measure. Then $E \int_{E_{0, T}} \mathbf{1}_{A}(\omega, z) \nu(d z)=0$ by the assumption on $A$. Therefore using Fubini's theorem we obtain

$$
0=\int_{E_{0, T}} E \mathbf{1}_{A}(\omega, z) \nu(d z)=\int_{E_{0, T}} P\left(A_{z}\right) \nu(d z) .
$$

Since $\nu$ was arbitrary, it follows from [28, Theorem 2.5] (or remark at the end of Section 4 in [18]) that $P\left(A_{z}\right)=0$ for cap-q.e. $z \in E_{0, T}$.

Proposition 4.6. Assume that $u$ is given by (3.19). Then $u\left(\mathbf{X}_{t}\right)=Y_{t}, t \in\left[0, T_{\iota}\right]$ for q.e. $z \in E_{0, T}$, where $(Y, M)$ is a solution of $\operatorname{BDSDE}_{z}(\varphi, f, g)$. Moreover, $M$ is a random martingale $A F$ of $\mathbf{M}^{0, T}$ and

$$
E e(M)=E\left(\|u(0)\|_{L^{2}(E ; m)}^{2}+\mathcal{B}^{0, T}(u, u)-\frac{1}{2} \int_{E_{0, T}}|u(z)|^{2} k(d z)\right),
$$

where $k$ is the killing measure of the form $\left(\hat{\mathcal{E}}^{0, T}, D\left(\hat{\mathcal{E}}^{0, T}\right)\right)$ defined in Lemma 4.3.

Proof. The first assertion follows from Theorem 3.7. If $g \equiv 0$ then (4.12) follows from [17, Lemma 6.1]. Therefore we may and will assume that $\varphi \equiv 0, f \equiv 0$. Let $u_{\alpha}=\alpha R_{\alpha}^{0, T} u$. Then

$$
u_{\alpha}\left(\mathbf{X}_{t}\right)=\int_{0}^{t} \alpha\left(u-u_{\alpha}\right)\left(\mathbf{X}_{r}\right) d r+\int_{0}^{t} d M_{r}^{\left[u_{\alpha}\right]}, \quad t \in\left[0, T_{\iota}\right] .
$$

By Itô's formula,

$$
\mathbb{E}_{z} \int_{0}^{T_{\iota}} d\left[M-M^{\left[u_{\alpha}\right]}\right]_{t} \leq \mathbb{E}_{z} \int_{0}^{T_{\iota}}\left(-2 \alpha\left|u-u_{\alpha}\right|^{2}\left(\mathbf{X}_{t}\right)+\left\|g\left(\mathbf{X}_{t}\right)\right\|^{2}\right) d t
$$

By Itô's isometry, for q.e. $z \in E_{0, T}$ we have

$$
\begin{aligned}
\alpha \mathbb{E}_{z} \int_{0}^{T_{\iota}}\left|u-u_{\alpha}\right|^{2}\left(\mathbf{X}_{t}\right) d t & =\alpha \mathbb{E}_{z} \int_{0}^{T_{\iota}}\left(E_{\mathbf{X}_{t}} \int_{0}^{T_{\iota}} e^{-\alpha r} g\left(\mathbf{X}_{r}\right) d^{\dagger} \beta_{r}^{\iota}\right)^{2} d t \\
& =\alpha \mathbb{E}_{z} \int_{0}^{T_{\iota}} E_{\mathbf{X}_{t}} \int_{0}^{T_{\iota}} e^{-2 \alpha r}\left\|g\left(\mathbf{X}_{r}\right)\right\|^{2} d r d t \\
& =\frac{1}{2} \mathbb{E}_{z} \int_{0}^{T_{\iota}} 2 \alpha R_{2 \alpha}^{0, T}\|g\|^{2}\left(\mathbf{X}_{t}\right) d t .
\end{aligned}
$$


This implies that

$$
2 \alpha \mathbb{E}_{z} \int_{0}^{T_{\iota}}\left|u-u_{\alpha}\right|^{2}\left(\mathbf{X}_{t}\right) d t \rightarrow \mathbb{E}_{z} \int_{0}^{T_{\iota}}\left\|g\left(\mathbf{X}_{t}\right)\right\|^{2} d t
$$

because

$$
\begin{aligned}
\mathbb{E}_{z} \int_{0}^{T_{\iota}} 2 \alpha R_{2 \alpha}^{0, T}\|g\|^{2}\left(\mathbf{X}_{t}\right) d t & =E R^{0, T}\left(2 \alpha R_{2 \alpha}^{0, T}\|g\|^{2}\right)(z)=E R^{0, T}\|g\|^{2}(z)-R_{2 \alpha}^{0, T}\|g\|^{2}(z) \\
& =\mathbb{E}_{z} \int_{0}^{T_{\iota}}\left\|g\left(\mathbf{X}_{t}\right)\right\|^{2} d t-\mathbb{E}_{z} \int_{0}^{T_{\iota}} e^{-2 \alpha t}\left\|g\left(\mathbf{X}_{t}\right)\right\|^{2} d t
\end{aligned}
$$

and by Lemma 3.2, $\mathbb{E}_{z} \int_{0}^{T_{\iota}}\left\|g\left(\mathbf{X}_{t}\right)\right\|^{2} d t<\infty$ for q.e. $z \in E_{0, T}$. By (4.13) and (4.14),

$$
\mathbb{E}_{z} \int_{0}^{T_{\iota}} d\left[M-M^{\left[u_{\alpha}\right]}\right]_{t} \rightarrow 0
$$

for q.e. $z \in E_{0, T}$. From (4.15), Burkholder-Davis-Gundy inequality and Lemma 4.4 we conclude that there exists a process $\tilde{M}$ such that, up to a subsequence, for $P$-a.e. $\omega \in \Omega$,

$$
E_{z} \sup _{t \leq T_{\iota}}\left|M_{t}^{\left[u_{\alpha}\right]}-\tilde{M}_{t}\right| \rightarrow 0
$$

for q.e. $z \in E_{0, T}$. By a standard argument (see the reasoning following Eq. (5.2.23) in the proof of $\left[8\right.$, Theorem 5.2.1]), $\tilde{M}(\omega ; \cdot)$ is a martingale $\mathrm{AF}$ of $\mathbf{M}^{0, T}$ for $P$-a.e. $\omega \in \Omega$. By Lemma 4.5, (4.15) and (4.16), $\tilde{M}$ is a $\mathbb{P}_{z}$-modification of $M$ for q.e. $z \in E_{0, T}$. By Proposition 4.1 and Lemma $4.2, u_{\alpha} \rightarrow u$ in $M^{2}(0, T ; V)$ and $E\left|u_{\alpha}(t)-u(t)\right|_{L^{2}(E ; m)}^{2} \rightarrow 0$ for every $t \in[0, T)$ as $\alpha \rightarrow \infty$. Write $M^{\alpha}=M^{\left[u_{\alpha}\right]}$. Let $\alpha_{n} \rightarrow \infty$. By Lemma 4.3,

$$
E e\left(M^{\alpha_{n}}-M^{\alpha_{m}}\right) \leq E\left\|\left(u_{\alpha_{n}}-u_{\alpha_{m}}\right)(0)\right\|_{L^{2}(E ; m)}^{2}+E \mathcal{B}^{0, T}\left(u_{\alpha_{n}}-u_{\alpha_{m}}, u_{\alpha_{n}}-u_{\alpha_{m}}\right),
$$

which converges to zero as $n, m \rightarrow \infty$. From this and (4.15) it follows that

$$
\lim _{n \rightarrow \infty} E e\left(M^{\alpha_{n}}\right)=E e(M) .
$$

By Lemma 4.3,

$$
E e\left(M^{\alpha}\right)=E\left\|u_{\alpha}(0)\right\|_{L_{2}(E ; m)}^{2}+E \mathcal{B}^{0, T}\left(u_{\alpha}, u_{\alpha}\right)-\frac{1}{2} E \int_{E_{0, T}}\left|u_{\alpha}(z)\right|^{2} k(d z) .
$$

Observe now that

$$
E \int_{E_{0, T}}\left|u_{\alpha}(z)\right|^{2} k(d z) \rightarrow E \int_{E_{0, T}}|u(z)|^{2} k(d z) .
$$

Indeed, since from the proof of Lemma 4.3 we know that (4.8) holds for quasi-continuous elements of $\mathcal{W}_{T}$, there exists $v \in L^{2}\left(E_{0, T} ; k\right)$ such that $\left(E\left|u_{\alpha}\right|^{2}\right)^{1 / 2} \rightarrow v$ in $L^{2}\left(E_{0, T} ; k\right)$. Therefore the proof of (4.18) is completed by showing that $v^{2}=E u^{2} k$-a.e. By (4.2),

$$
E\left|u(z)-u_{\alpha}(z)\right|^{2} \leq \mathbb{E}_{z} \int_{0}^{T_{\iota}} e^{-2 \alpha t} \| g\left(\mathbf{X}_{t} \|^{2} d t, \quad z \in E_{0, T}\right.
$$

By Lemma 3.2, $\mathbb{E}_{z} \int_{0}^{T_{\iota}}\left\|g\left(\mathbf{X}_{t}\right)\right\|^{2} d t<\infty$ for q.e. $z \in E_{0, T}$. Hence $E u_{\alpha}^{2} \rightarrow E u^{2}$ q.e. Consequently, $E u_{\alpha}^{2} \rightarrow E u^{2} k$-a.e. since $k$ is smooth. Thus $v^{2}=E u^{2}$, which completes the proof of (4.18). Letting $\alpha \rightarrow \infty$ in (4.17) and using (4.18) we get (4.12). 
Remark 4.7. Let $N$ be defined by (1.8). A direct calculation shows that

$$
e(N)=\frac{1}{2}\left\|g_{u}\right\|_{L^{2}\left(E_{0, T} ; m_{T}\right)}^{2} .
$$

From this one can conclude that $u \notin \mathbb{W}$, where $\mathbb{W}$ is defined by (1.10). Indeed, suppose that $u \in \mathbb{W}$. We may assume that $\varphi=0, f=0$ and $g$ does not depend on $y$. Let $A^{[u]}$ be defined by (1.7) and $u_{\alpha}$ be as in the proof of Proposition 4.6. Then by the proof of [8, Theorem 5.2.2] (see also [28, Theorem 4.5]), $N=N^{[u]}$ and $N^{\left[u_{\alpha}\right]}$ converges to $N$ uniformly on compacts in probability $\mathbb{P}_{m_{T}}$. Since $N=N^{[u]}$ we have

$$
\begin{aligned}
E e(N) & \leq 2 E e\left(N-N^{\left[u_{\alpha}\right]}\right)+2 E e\left(A^{\left[u_{\alpha}\right]}\right)=2 E e\left(N-N^{\left[u_{\alpha}\right]}\right) \\
& \leq 4 E e\left(A^{[u]}-A^{\left[u_{\alpha}\right]}\right)+4 E e\left(M^{[u]}-M^{\left[u_{\alpha}\right]}\right) .
\end{aligned}
$$

Since $u \in \mathbb{W}$, we have

$$
\begin{aligned}
E e\left(A^{[u]}-A^{\left[u_{\alpha}\right]}\right) & \leq E \mathcal{E}\left(u_{\alpha}-u, u_{\alpha}-u\right) \\
& =E\left\|u_{\alpha}(0)-u(0)\right\|_{L^{2}(E ; m)}^{2}+E \mathcal{B}^{0, T}\left(u_{\alpha}-u, u_{\alpha}-u\right) .
\end{aligned}
$$

(see, e.g., [28, Eq. (13)]). When combined with the previous inequality and Lemma 4.3 this shows that $e(N)=0$, which contradicts (4.19).

In what follows by $\mathcal{M}^{+}$we denote the set of all positive Borel measures on $E_{0, T}$. By $\mathcal{M}_{0, b}^{+}$we denote the subset of $\mathcal{M}^{+}$consisting of all bounded measures which charge no set of zero capacity associated with the form $\left(\mathcal{E}^{0, T}, D\left(\mathcal{E}^{0, T}\right)\right)$. The total variation norm of $\mu \in \mathcal{M}_{0, b}^{+}$will be denoted by $\|\mu\|_{T V}$. To shorten notation, for $\mu \in \mathcal{M}^{+}$we write $P_{\mu}(\cdot)=\int_{E_{0, T}} P_{z}(\cdot) \mu(d z)$ and by $E_{\mu}$ (resp. $\mathbb{E}_{\mu}$ ) we denote the expectation with respect to $P_{\mu}$ (resp. $\left.P \otimes P_{\mu}\right)$.

Definition. We say that $\mu: \Omega \times \mathcal{B}\left(E_{0, T}\right) \rightarrow \mathbb{R}$ is a random measure if $\mu(\omega ; \cdot)$ is a positive Radon measure on $E_{0, T}$ for $P$-a.s. $\omega \in \Omega$ and $\mu(\omega ; B)$ is $\mathcal{F}_{T}^{\beta}$-measurable for every $B \in \mathcal{B}\left(E_{0, T}\right)$.

Given a random measure $\mu$ such that $\mu(\omega ; \cdot) \in \mathcal{M}_{0, b}^{+}$for $P$-a.e. $\omega \in \Omega$ we denote by $A^{\mu}$ the random positive $\mathrm{AF}$ of $\mathbf{M}^{0, T}$ such that for $P$-a.e. $\omega \in \Omega$ the measure $\mu(\omega ; \cdot)$ is the Revuz measure of the $\mathrm{AF} A(\omega ; \cdot)$. We call $A^{\mu}$ the random AF associated with $\mu$. Note that if the random AF associated with $\mu$ exists, then it is uniquely determined.

In the rest of the paper writing $A^{\mu}$ for some random measure $\mu$ we tacitly assume that this random AF exists.

Lemma 4.8. Assume that $\mu, \nu$ are random measures such that $\mu, \nu \in \mathcal{M}_{0, b}^{+}\left(E_{0, T}\right)$, $P$-a.s. If for some $v \in M^{2}(0, T ; V) \cap C\left([0, T] ; L^{2}(\Omega \times E ; P \otimes m)\right)$,

$$
E v(z)+\mathbb{E}_{z} \int_{0}^{T_{\iota}} d A_{t}^{\mu} \leq \mathbb{E}_{z} \int_{0}^{T_{\iota}} d A_{t}^{\nu}
$$

for $m_{T}$-a.e. $z \in E_{0, T}$ then

$$
E \int_{[0, T) \times E} v(z) k(d z)+E\|\mu\|_{T V} \leq E\|\nu\|_{T V} .
$$


Proof. Since $E v(z)+E R^{0, T} \mu(z) \leq E R^{0, T} \nu(z)$ for $m_{T^{-}}$a.e. $z \in E_{0, T}$, we have

$$
\begin{aligned}
& E\left(v, \alpha\left(1-\alpha \hat{R}_{\alpha}^{0, T} 1\right)\right)_{L^{2}\left(E_{0, T} ; m_{T}\right)}+E\left(R^{0, T} \mu, \alpha\left(1-\alpha \hat{R}_{\alpha}^{0, T} 1\right)\right)_{L^{2}\left(E_{0, T} ; m_{T}\right)} \\
& \quad \leq E\left(R^{0, T} \nu, \alpha\left(1-\alpha \hat{R}_{\alpha}^{0, T} 1\right)\right)_{L^{2}\left(E_{0, T} ; m_{T}\right)} .
\end{aligned}
$$

Hence

$$
\begin{aligned}
& E\left(v, \alpha\left(1-\alpha \hat{R}_{\alpha}^{0, T} 1\right)\right)_{L^{2}\left(E_{0, T} ; m_{T}\right)}+E\left(\alpha\left(I-\alpha R_{\alpha}^{0, T}\right) R^{0, T} \mu, 1\right)_{L^{2}\left(E_{0, T} ; m_{T}\right)} \\
& \quad \leq E\left(\alpha\left(I-\alpha R_{\alpha}^{0, T}\right) R^{0, T} \nu, 1\right)_{L^{2}\left(E_{0, T} ; m_{T}\right)} .
\end{aligned}
$$

It is an elementary check that

$$
\alpha\left(I-\alpha R_{\alpha}^{0, T}\right) R^{0, T} \mu(z)=E_{z} \int_{0}^{T_{\iota}} \alpha e^{-\alpha t} d A_{t}^{\mu}, \quad z \in E_{0, T}
$$

Therefore

$$
E\left(v, \alpha\left(1-\alpha \hat{R}_{\alpha}^{0, T} 1\right)\right)_{L^{2}\left(E_{0, T} ; m_{T}\right)}+\mathbb{E}_{m_{T}} \int_{0}^{T_{\iota}} \alpha e^{-\alpha t} d A_{t}^{\mu} \leq \mathbb{E}_{m_{T}} \int_{0}^{T_{\iota}} \alpha e^{-\alpha t} d A_{t}^{\nu} .
$$

Letting $\alpha \rightarrow \infty$ in (4.21) we get (4.20). Indeed, directly from the definition of the Revuz duality it follows that the integrals involving $A^{\mu}$ and $A^{\nu}$ converge to $E\left\|_{\mu}\right\|_{T V}$ and $E\|\nu\|_{T V}$, respectively. To show the convergence of the first term on the left-hand side of (4.21), let us first assume that $v(T)=0$. Set $v_{m}=\beta_{m} R_{\beta_{m}}^{0, T} v$. Then by (4.10),

$$
\begin{aligned}
\limsup _{n \rightarrow \infty}\left|\left(v^{2}, k^{\alpha_{n}}\right)^{1 / 2}-\left(v^{2}, k\right)^{1 / 2}\right| \leq & 4\left\|\left(v_{m}-v, v_{m}-v\right)(0)\right\|_{L_{2}(E ; m)} \\
& +4\left(\mathcal{B}^{0, T}\left(v_{m}-v, v_{m}-v\right)\right)^{1 / 2} .
\end{aligned}
$$

Since $v_{m}(t) \rightarrow v(t)$ in $L^{2}(E ; m)$ for every $t \in[0, T)$ and $v_{m} \rightarrow v$ in $M^{2}(0, T ; V)$, this shows the desired convergence of the first term. In general, if $v(T) \neq 0$, we consider a sequence $\left\{v_{n}\right\} \subset M^{2}(0, T ; V) \cap C\left([0, T] ; L^{2}(\Omega \times E ; P \otimes m)\right)$ such that $v_{n} \leq v, v_{n}(T)=0$ and $v_{n} \nearrow v$ on $[0, T) \times E$. Of course, $v_{n}$ satisfies the assumptions of the lemma, so by what has already been proved, (4.20) is satisfied with $v$ replaced by $v_{n}$. Letting $n \rightarrow \infty$ we get (4.20) for $v$.

Theorem 4.9. Assume (H1)-(H3). Let $u$ be a solution of SPDE (3.4). Then $u \in$ $S^{2}\left(0, T ; L^{2}(E ; m)\right) \cap M^{2}(0, T ; V)$ and there is $c>0$ depending only on $T, L, l$ such that

$$
\begin{aligned}
& \sup _{0 \leq t \leq T} E\|u(t)\|_{L^{2}(E ; m)}^{2}+E \mathcal{B}^{0, T}(u, u) \\
& \quad \leq c E\left(\|\varphi\|_{L^{2}(E ; m)}^{2}+\|f(\cdot, 0)\|_{L^{2}\left(E_{0, T} ; m_{T}\right)}^{2}+\sum_{k=1}^{\infty}\left\|g_{k}(\cdot, 0)\right\|_{L^{2}\left(E_{0, T} ; m_{T}\right)}^{2}\right) .
\end{aligned}
$$

Proof. By Proposition 2.5,

$$
\begin{aligned}
\mathbb{E}_{z} & \sup _{0 \leq t \leq T_{\iota}}\left|u\left(\mathbf{X}_{t}\right)\right|^{2}+\mathbb{E}_{z} \int_{0}^{T_{\iota}} d[M]_{t} \\
& \leq c \mathbb{E}_{z}\left(\left|\varphi\left(\mathbf{X}_{T_{\iota}}\right)\right|^{2}+\int_{0}^{T_{\iota}}\left(\left|f\left(\mathbf{X}_{t}, 0\right)\right|^{2}+\left\|g\left(\mathbf{X}_{t}, 0\right)\right\|^{2}\right) d t\right)
\end{aligned}
$$


Hence

$$
E|u(z)|^{2}+\mathbb{E}_{z} \int_{0}^{T_{\iota}} d\langle M\rangle_{t} \leq c \mathbb{E}_{z} \int_{0}^{T_{\iota}} d A_{t}^{\nu}
$$

where

$$
\nu(d z)=\left(\delta_{\{T\}} \otimes|\varphi|^{2} \cdot m\right)(d z)+\left(|f(z, 0)|^{2}+\|g(z, 0)\|^{2}\right) m_{T}(d z) .
$$

Let $\mu_{\langle M\rangle}$ denote the random smooth measure associated with the random continuous $\mathrm{AF}\langle M\rangle$ of M. By Lemma 4.8,

$$
E \int_{E_{0, T}}|u(z)|^{2} k(d z)+E\left\|\mu_{\langle M\rangle}\right\|_{T V} \leq c E\|\nu\|_{T V}
$$

Since by (4.4), $\left\|\mu_{\langle M\rangle}\right\|_{T V}=2 e(M), P$-a.s., it follows from Proposition 4.6 and (4.23) that

$$
E\|u(0)\|_{L^{2}(E ; m)}^{2}+E \mathcal{B}^{0, T}(u, u) \leq c E\|\nu\|_{T V} .
$$

Since the same estimate can be obtained on any interval $[t, T]$ with $t \in(0, T)$, and $c E\|\nu\|_{T V}$ is equal to the right-hand side of (4.22), the theorem is proved.

\section{BDSDEs with Brownian filtration}

In the present section and in Section 6 we assume that the filtration $\left(\mathcal{G}_{t}\right)$ of Section 2 is generated by a $d$-dimensional Wiener process $W$ on $\Omega^{\prime}$. This will allow us to treat in Section 6 equations dependent on the gradient of a solution.

We also assume that we are given an $\mathcal{F}_{T}$-measurable random variable $\xi$ and two families $\{f(t, y, z), t \geq 0\}_{y \in \mathbb{R}, z \in \mathbb{R}^{d}},\left\{g_{k}(t, y, z), t \geq 0\right\}_{y \in \mathbb{R}, z \in \mathbb{R}^{d}, k \in \mathbb{N}}$ of processes of class $M$ (as in Section 2, in our notation we omit the dependence on $\left.\left(\omega, \omega^{\prime}\right) \in \Omega \times \Omega^{\prime}\right)$. We set $g(\cdot, y, z)=\left(g_{1}(\cdot, y, z), g_{2}(\cdot, y, z), \ldots\right)$.

Let us consider the following hypotheses.

(B1) $\mathbb{E}|\xi|^{2}+\mathbb{E} \int_{0}^{T}|f(t, 0,0)|^{2} d t+\mathbb{E} \int_{0}^{T}\|g(t, 0,0)\|^{2} d t<\infty$.

(B2) $\int_{0}^{T}|f(t, y, 0)| d t<\infty, \mathbb{P}$-a.s. for every $y \in \mathbb{R}$.

(B3) There exist $l, L>0, m \in(0,1)$ and $M_{k}, L_{k}: \Omega \times[0, T] \rightarrow \mathbb{R}_{+}$such that $\sup _{0 \leq t \leq T} \sum_{k=1}^{\infty} L_{k}^{2}(t) \leq l, \sup _{0 \leq t \leq T} \sum_{k=1}^{\infty} M_{k}^{2}(t) \leq m, P$-a.s. and for a.e. $t \in$ $[0, T]$

(a) $\left(f(t, y, z)-f\left(t, y^{\prime}, z\right)\right)\left(y-y^{\prime}\right) \leq L\left|y-y^{\prime}\right|^{2}$ for all $y, y^{\prime} \in \mathbb{R}, z \in \mathbb{R}^{d}$,

(b) $\left|f(t, y, z)-f\left(t, y, z^{\prime}\right)\right| \leq L\left|z-z^{\prime}\right|$ for all $y \in \mathbb{R}, z, z^{\prime} \in \mathbb{R}^{d}$,

(c) $\left|g_{k}(t, y, z)-g_{k}\left(t, y^{\prime}, z^{\prime}\right)\right| \leq L_{k}(t)\left|y-y^{\prime}\right|+M_{k}(t)\left|z-z^{\prime}\right|$ for all $y, y^{\prime} \in \mathbb{R}$, $z, z^{\prime} \in \mathbb{R}^{d}$.

(B4) For a.e. $t \in[0, T]$ and every $z \in \mathbb{R}^{d}$ the mapping $\mathbb{R} \ni y \mapsto f(t, y, z)$ is continuous.

Definition. We say that a pair $(Y, Z) \in \mathcal{S}^{2} \times M^{2}$ is a solution of $\operatorname{BDSDE}(\xi, f, g)$ if

(a) $\mathbb{P}\left(\int_{0}^{T}\left(\left|f\left(t, Y_{t}, 0\right)\right|+\left\|g\left(t, Y_{t}, 0\right)\right\|^{2}\right) d t<\infty\right)=1$,

(b) $Y_{t}=\xi+\int_{t}^{T} f\left(r, Y_{r}, Z_{r}\right) d r+\int_{t}^{T} g\left(r, Y_{r}, Z_{r}\right) d^{\dagger} \beta_{r}-\int_{t}^{T} Z_{r} d W_{r}, t \in[0, T]$, P-a.s. 
Remark 5.1. If the coefficients $f, g$ do not depend on $z$ and $(Y, Z)$ is a solution of $\operatorname{BDSDE}(\xi, f, g)$ then the pair

$$
\left(Y_{t}, M_{t}\right):=\left(Y_{t}, \int_{0}^{t} Z_{r} d W_{r}\right), \quad t \in[0, T]
$$

is a solution of $\operatorname{BDSDE}(\xi, f, g)$ in the sense of Section 3. The difference between Section 3 and Section 5 is that in the present section we have additional information on the filtration $\left(\mathcal{G}_{t}\right)$, which gives us additional information on the component $M$ of the solution.

Proposition 5.2. Let $g$ satisfy (B3c) and either $f$ or $f^{\prime}$ satisfy (B3a), (B3b). If $\xi \leq \xi^{\prime}$, $\mathbb{P}$-a.s. and $f^{\prime}(t, y, z) \leq f(t, y, z)$ for a.e. $t \in[0, T]$ and every $y \in \mathbb{R}$ and $z \in \mathbb{R}^{d}$ then

$$
Y_{t}^{\prime} \leq Y_{t}, \quad t \in[0, T], \quad \mathbb{P} \text {-a.s. },
$$

where $(Y, M),\left(Y^{\prime}, M^{\prime}\right)$ are solutions of $\operatorname{BDSDE}(\xi, f, g)$ and $\operatorname{BSDE}\left(\xi^{\prime}, f^{\prime}, g\right)$, respectively.

Proof. Assume that $f$ satisfies (B3a), (B3b). By Itô's formula,

$$
\begin{aligned}
\left|\left(Y_{t}^{\prime}-Y_{t}\right)^{+}\right|^{2}+\int_{t}^{T} \mathbf{1}_{\left\{Y_{r}^{\prime}>Y_{r}\right\}}\left|Z_{r}^{\prime}-Z_{r}\right|^{2} d r \\
\leq 2 \int_{t}^{T}\left(Y_{r}^{\prime}-Y_{r}\right)^{+}\left(f^{\prime}\left(r, Y_{r}^{\prime}, Z_{r}^{\prime}\right)-f\left(r, Y_{r}, Z_{r}\right)\right) d r-2 \int_{t}^{T}\left(Y_{r}^{\prime}-Y_{r}\right)^{+}\left(Z_{r}^{\prime}-Z_{r}\right) d W_{r} \\
\quad+\sum_{k=1}^{\infty} \int_{t}^{T} \mathbf{1}_{\left\{Y_{r}^{\prime}>Y_{r}\right\}}\left|g_{k}\left(r, Y_{r}^{\prime}, Z_{r}^{\prime}\right)-g_{k}\left(r, Y_{r}, Z_{r}\right)\right|^{2} d r .
\end{aligned}
$$

By the assumptions,

$$
\int_{t}^{T}\left(Y_{r}^{\prime}-Y_{r}\right)^{+}\left(f^{\prime}\left(r, Y_{r}^{\prime}, Z_{r}^{\prime}\right)-f\left(r, Y_{r}, Z_{r}\right)\right) d r \leq 2 L \int_{t}^{T}\left|\left(Y_{r}^{\prime}-Y_{r}\right)^{+}\right|^{2} d r
$$

and

$$
\begin{aligned}
& \sum_{k=1}^{\infty} \int_{t}^{T} \mathbf{1}_{\left\{Y_{r}^{\prime}>Y_{r}\right\}}\left|g_{k}\left(r, Y_{r}^{\prime}, Z_{r}^{\prime}\right)-g_{k}\left(r, Y_{r}, Z_{r}\right)\right|^{2} d r \\
& \quad \leq \sum_{k=1}^{\infty} \int_{t}^{T}\left(L_{k}^{2}(r) \mathbf{1}_{\left\{Y_{r}^{\prime}>Y_{r}\right\}}\left|Y_{r}^{\prime}-Y_{r}\right|^{2}+M_{k}^{2}(r) \mathbf{1}_{\left\{Y_{r}^{\prime}>Y_{r}\right\}}\left|Z_{r}^{\prime}-Z_{r}\right|^{2}\right) d r \\
& \quad \leq l \int_{t}^{T}\left|\left(Y_{r}^{\prime}-Y_{r}\right)^{+}\right|^{2} d r+m \int_{t}^{T} \mathbf{1}_{\left\{Y_{r}^{\prime}>Y_{r}\right\}}\left|Z_{r}^{\prime}-Z_{r}\right|^{2} d r .
\end{aligned}
$$

By the above estimates,

$$
\mathbb{E}\left|\left(Y_{t}^{\prime}-Y_{t}\right)^{+}\right|^{2} \leq 2(L+l) \mathbb{E} \int_{t}^{T}\left|\left(Y_{r}^{\prime}-Y_{r}\right)^{+}\right|^{2} d r, \quad t \in[0, T],
$$

so Gronwall's lemma yields the desired result. 
Corollary 5.3. Let assumption (B3) hold. Then there exists at most one solution of $\operatorname{BDSDE}(\xi, f, g)$.

Theorem 5.4. Let assumptions (B1)-(B4) hold. Then there exists a solution $(Y, Z)$ of $\operatorname{BDSDE}(\xi, f, g)$.

Proof. Let $M_{k}^{2}$ denote the set of $k$-dimensional processes $X=\left(X^{1}, \ldots, X^{k}\right)$ such that $X^{i} \in M^{2}, i=1, \ldots, k$. Define the mapping $\Phi: M_{1}^{2} \otimes M_{d}^{2} \rightarrow M_{1}^{2} \otimes M_{d}^{2}$ by letting $\Phi(U, V)$ be the solution $(Y, Z)$ of the BSDE

$$
Y_{t}=\xi+\int_{t}^{T} f\left(r, Y_{r}, V_{r}\right) d r+\int_{t}^{T} g\left(r, Y_{r}, V_{r}\right) d^{\dagger} B_{r}-\int_{t}^{T} Z_{r} d W_{r}, \quad t \in[0, T] .
$$

From Corollary 5.3, Theorem 2.6 and the representation theorem for Brownian filtration it follows that $\Phi$ is well defined. Let $\left(U^{1}, V^{1}\right),\left(U^{2}, V^{2}\right) \in M_{1}^{2} \otimes M_{d}^{2}$ and $\left(Y^{i}, Z^{i}\right)=$ $\Phi\left(U^{i}, V^{i}\right), i=1,2$. By Itô's formula,

$$
\begin{aligned}
\mathbb{E} e^{\beta t}\left|Y_{t}^{1}-Y_{t}^{2}\right|^{2}+\beta \mathbb{E} \int_{t}^{T} e^{\beta r}\left|Y_{r}^{1}-Y_{r}^{2}\right|^{2} d r+\mathbb{E} \int_{t}^{T} e^{\beta r}\left|Z_{r}^{1}-Z_{r}^{2}\right|^{2} d r \\
=2 \mathbb{E} \int_{t}^{T} e^{\beta r}\left(f\left(r, Y_{r}^{1}, V_{r}^{1}\right)-f\left(r, Y_{r}^{2}, V_{r}^{2}\right)\right)\left(Y_{r}^{1}-Y_{r}^{2}\right) d r \\
\quad+\mathbb{E} \int_{t}^{T} e^{\beta r}\left\|g\left(r, Y_{r}^{1}, V_{r}^{1}\right)-g\left(r, Y_{r}^{2}, V_{r}^{2}\right)\right\|^{2} d r \\
\leq 2 L \mathbb{E} \int_{t}^{T} e^{\beta r}\left(\left|Y_{r}^{1}-Y_{r}^{2}\right|^{2}+\left|V_{r}^{1}-V_{r}^{2}\right| \cdot\left|Y_{r}^{1}-Y_{r}^{2}\right|\right) d r \\
\quad+l \mathbb{E} \int_{t}^{T} e^{\beta r}\left|Y_{r}^{1}-Y_{r}^{2}\right|^{2} d r+m E \int_{t}^{T}\left|V_{r}^{1}-V_{r}^{2}\right|^{2} d r
\end{aligned}
$$

Hence, for every $\alpha>0$,

$$
c \mathbb{E} \int_{t}^{T} e^{\beta r}\left|Y_{r}^{1}-Y_{r}^{2}\right|^{2} d r+\mathbb{E} \int_{t}^{T} e^{\beta r}\left|Z_{r}^{1}-Z_{r}^{2}\right|^{2} d r \leq(\alpha+m) \mathbb{E} \int_{t}^{T}\left|V_{r}^{1}-V_{r}^{2}\right|^{2} d r
$$

with $c=\beta-2 L-(2 L)^{2} \alpha^{-1}-l>0$. Let $\alpha, \beta$ be chosen so that $c>0$ and $\alpha+m<1$. Then $\Phi$ is a contraction if we equip $M_{1}^{2} \times M_{d}^{2}$ with the norm

$$
\|(Y, Z)\|_{\beta}^{2}=\mathbb{E} \int_{0}^{T} e^{\beta r}\left(c\left|Y_{r}\right|^{2}+\left|Z_{r}\right|^{2}\right) d r .
$$

By Banach's principle, $\Phi$ has a fixed point. Of course, it solves $\operatorname{BDSDE}(\xi, f, g)$.

\section{SPDEs with divergence form operator}

In this section we consider equations of the form (1.1) with $A$ being a uniformly elliptic divergence form operator. We allow, however, the coefficients $f, g$ to depend on the gradient of a solution. More precisely, we assume that $E=D$ is an nonempty bounded open subset of $\mathbb{R}^{d}$ and

$$
B^{(t)}(\varphi, \psi)=\sum_{i, j=1}^{d} \int_{D} a_{i j}(t, x) \varphi_{x_{i}}(x) \psi_{x_{j}}(x) d x, \quad \varphi, \psi \in V=H_{0}^{1}(D),
$$


where $a_{i j}:[0, T] \times D \rightarrow \mathbb{R}$ are measurable functions such that for every $(t, x) \in$ $[0, T] \times D$

$$
a_{i j}=a_{j i}, \quad \lambda|\xi|^{2} \leq \sum_{i, j=1}^{d} a_{i j}(t, x) \xi_{i} \xi_{j} \leq \Lambda|\xi|^{2}, \quad \xi \in \mathbb{R}^{d}
$$

for some $0<\lambda \leq \Lambda$. In this case the operator $A_{t}$ associated with $\left(B^{(t)}, V\right)$ is given by (1.2). Suppose we are given measurable functions $\varphi: D \rightarrow \mathbb{R}$ and $f, g_{k}: \Omega \times[0, T] \times$ $D \times \mathbb{R} \times \mathbb{R}^{d} \rightarrow \mathbb{R}$. We consider equation of the form

$$
d u(t)=-\left(A_{t} u+f(t, x, u, \sigma \nabla u)\right) d t-g(t, x, u, \sigma \nabla u) d^{\dagger} \beta_{t}, \quad u(T)=\varphi,
$$

where $\sigma$ is such that $\sigma \cdot \sigma^{T}=a$. We are going to show that (6.1) has a unique solution under the following assumptions:

(D1) $E\|\varphi\|_{L^{2}(E ; m)}^{2}+E\|f(\cdot, 0,0)\|_{L^{2}\left(E_{0, T} ; m_{T}\right)}^{2}+E \sum_{k=1}^{\infty}\left\|g_{k}(\cdot, 0,0)\right\|_{L^{2}\left(E_{0, T} ; m_{T}\right)}^{2}<\infty$.

(D2) For all $y \in \mathbb{R}$ and $e \in \mathbb{R}^{d}$ the mapping $E_{0, T} \ni z \mapsto f(z, y, e)$ belongs to $q \mathbb{L}^{1}$.

(D3) There exist $l, L>0, m \in(0,1)$ and functions $M_{k}, L_{k}: E_{0, T} \rightarrow \mathbb{R}_{+}$such that $\sup _{z \in E_{0, T}} \sum_{k} L_{k}(r) \leq l, \sup _{z \in E_{0, T}} \sum_{k} M_{k}(r) \leq m$ and for every $z \in E_{0, T}$ we have

(a) $\left(f(z, y, e)-f\left(z, y^{\prime}, e\right)\right)\left(y-y^{\prime}\right) \leq L\left|y-y^{\prime}\right|^{2}$ for all $y, y^{\prime} \in \mathbb{R}, e \in \mathbb{R}^{d}$,

(b) $\left|f(z, y, e)-f\left(z, y, e^{\prime}\right)\right| \leq L\left|e-e^{\prime}\right|$ for all $y \in \mathbb{R}, e, e^{\prime} \in \mathbb{R}^{d}$,

(c) $\left|g_{k}(z, y, e)-g_{k}\left(z, y^{\prime}, e^{\prime}\right)\right| \leq L_{k}(z)\left|y-y^{\prime}\right|+M_{k}(z)\left|e-e^{\prime}\right|$ for all $y, y^{\prime} \in \mathbb{R}$, $e, e^{\prime} \in \mathbb{R}^{d}$.

(D4) For every $z \in E_{0, T}$ and $e \in \mathbb{R}^{d}$ the mapping $\mathbb{R} \ni y \mapsto f(z, y, e)$ is continuous.

(D5) For every $y \in \mathbb{R}, e \in \mathbb{R}^{d}$ the mappings $f(\cdot, y, e), g_{k}(\cdot, y, e): \Omega \times E_{T} \rightarrow \mathbb{R}, k \in \mathbb{N}$, are $\left(\mathcal{F}_{t, T}^{\beta}\right)$-progressively measurable.

The process $\mathbf{M}^{0, T}$ associated with the operator $\frac{\partial}{\partial t}-A_{t}$ has the following unique Fukushima decomposition

$$
\mathbf{X}_{t}=\mathbf{X}_{0}+\mathbf{M}_{t}+\mathbf{A}_{t}, \quad t \in\left[0, T_{\iota}\right], \quad P_{z} \text {-a.s. }, \quad z \in E_{0, T},
$$

where $\mathbf{M}$ is a martingale $\mathrm{AF}$ of $\mathbf{M}^{0, T}$ of finite energy and $\mathbf{A}$ is a continuous $\mathrm{AF}$ of $\mathbf{M}^{0, T}$ of zero energy. It is well known that $W$ defined as

$$
W_{t}=\int_{0}^{t} \sigma^{-1}\left(\mathbf{X}_{r}\right) d \mathbf{M}_{r}^{\pi}, \quad t \geq 0,
$$

where $\mathbf{M}^{\pi}=\pi(\mathbf{M})$ and $\pi\left(x_{1}, \ldots, x_{d+1}\right)=\left(x_{2}, \ldots, x_{d+1}\right)$ for $x_{i} \in \mathbb{R}, i=1, \ldots, d+1$, is a standard $\left(\mathcal{G}_{t}\right)$-Brownian motion under $P_{z}$.

Proposition 6.1. Assume that $\varphi, f, g$ satisfy (D1)-(D5) and $f, g$ do not depend on the last variable e. Let $(Y, M)$ be the pair of process of Theorem 3.3 and let $u$ be the function defined by (3.19). Then $u \in M^{2}\left(0, T ; H_{0}^{1}(D)\right)$ and for q.e. $z \in E_{0, T}$,

$$
\left(u\left(\mathbf{X}_{t}\right), \int_{0}^{t} \sigma \nabla u\left(\mathbf{X}_{r}\right) d W_{r}\right)=\left(Y_{t}, M_{t}\right), \quad t \in\left[0, T_{\iota}\right], \quad \mathbb{P}_{z} \text {-a.s. }
$$


Proof. That $Y_{t}=u\left(\mathbf{X}_{t}\right), t \in\left[0, T_{\iota}\right]$, follows from Theorem 3.7. Observe that $M=M^{1}+$ $M^{2}$, where $M^{1}, M^{2}$ are processes of Theorem 3.3 associated with the data $(\varphi, f(\cdot, u), 0)$ and $(0,0, g(\cdot, u))$, respectively. The desired representation for $M^{1}$ follows from [11, Proposition 3.6]. Therefore we may and will assume that $\varphi=0, f=0$ and $g$ does not depend on $y$. By Theorem 4.9, $u \in M^{2}\left(0, T ; H_{0}^{1}(D)\right)$. Let $u_{\alpha}$ be defined as in the proof of Proposition 4.6. Then by (4.15) and the Burkholder-Davis-Gundy inequality,

$$
\lim _{\alpha \rightarrow \infty} \mathbb{E}_{z} \sup _{0 \leq t \leq T_{\iota}}\left|M_{t}-M_{t}^{\left[u_{\alpha}\right]}\right|^{2}=0 .
$$

On the other hand, it is well known (see [28, Theorem 5.6]) that

$$
M_{t}^{\left[u_{\alpha}\right]}=\int_{0}^{t} \sigma \nabla u_{\alpha}\left(\mathbf{X}_{r}\right) d W_{r}, \quad t \in\left[0, T_{\iota}\right]
$$

Hence

$$
\begin{aligned}
\mathbb{E}_{\nu} \sup _{0 \leq t \leq T_{\iota}}\left|M_{t}^{\left[u_{\alpha}\right]}-\int_{0}^{t} \sigma \nabla u\left(\mathbf{X}_{r}\right) d W_{r}\right|^{2} & \leq 4 \Lambda \mathbb{E}_{\nu} \int_{0}^{T_{\iota}}\left|\nabla u-\nabla u_{\alpha}\right|^{2}\left(\mathbf{X}_{t}\right) d t \\
& \leq 4 \Lambda E\left\|\nabla u-\nabla u_{\alpha}\right\|_{L^{2}\left(E_{0, T} ; m_{T}\right)}^{2}\left\|\hat{R}^{0, T} \nu\right\|_{\infty}
\end{aligned}
$$

for every $\nu \in \hat{S}_{00}$ (for the definition of $\hat{S}_{00}$ see Remark 3.1). Since $u_{\alpha} \rightarrow u$ in $M^{2}\left(0, T ; H_{0}^{1}(D)\right)$ (see Lemma 4.2), using standard arguments (see the reasoning in the proof of $[8$, Theorem 5.2.1]) we conclude from the above inequality and [28, Theorem 2.5] (see also the remark at the end of Section 4 in [18]) that, up to a subsequence,

$$
\lim _{\alpha \rightarrow \infty} \mathbb{E}_{z} \sup _{0 \leq t \leq T_{\iota}}\left|M_{t}^{\left[u_{\alpha}\right]}-\int_{0}^{t} \sigma \nabla u\left(\mathbf{X}_{r}\right) d W_{r}\right|^{2}=0
$$

for q.e. $z \in E_{0, T}$. When combined with (6.2) this completes the proof.

Definition. We say that a measurable function $u: E_{0, T} \rightarrow \mathbb{R}$ is a solution of (6.1) if

(a) $\mathbb{P}_{z}\left(\int_{0}^{T_{\iota}}\left|f\left(\mathbf{X}_{t}, u\left(\mathbf{X}_{t}\right), 0\right)\right| d t<\infty\right)=1, \mathbb{E}_{z} \sup _{0 \leq t \leq T_{\iota}}\left|\int_{0}^{t} f\left(\mathbf{X}_{r}, u\left(\mathbf{X}_{r}\right), 0\right) d r\right|^{2}<\infty$ and $\mathbb{E}_{z} \int_{0}^{T_{\iota}}\left\|g\left(\mathbf{X}_{t}, u\left(\mathbf{X}_{t}\right), 0\right)\right\|^{2} d t<\infty$ for q.e. $z \in E_{0, T}$,

(b) $u$ is quasi-continuous, $u \in M^{2}\left(0, T ; H_{0}^{1}(D)\right)$ and for q.e. $z \in E_{0, T}$,

$$
\begin{array}{r}
u(z)=E_{z}\left(\varphi\left(\mathbf{X}_{T_{\iota}}\right)+\int_{0}^{T_{\iota}} f\left(\mathbf{X}_{t}, u\left(\mathbf{X}_{t}\right), \sigma \nabla u\left(\mathbf{X}_{t}\right)\right) d t\right. \\
\left.+\int_{0}^{T_{\iota}} g\left(\mathbf{X}_{t}, u\left(\mathbf{X}_{t}\right), \sigma \nabla u\left(\mathbf{X}_{t}\right)\right) d^{\dagger} \beta_{t}^{\iota}\right) .
\end{array}
$$

Definition. A pair $\left(Y^{z}, Z^{z}\right) \in \mathcal{S}^{2} \times M^{2}$ is a solution of the BDSDE

$$
\begin{aligned}
Y_{t}^{z}= & \varphi\left(\mathbf{X}_{T_{\iota}}\right)+\int_{t}^{T_{\iota}} f\left(\mathbf{X}_{r}, Y_{r}^{z}, Z_{r}^{z}\right) d r \\
& +\int_{t}^{T_{\iota}} g\left(\mathbf{X}_{r}, Y_{r}^{z}, Z_{r}^{z}\right) d^{\dagger} \beta_{r}^{\iota}-\int_{0}^{T_{\iota}} Z_{r}^{z} d W_{r}, \quad t \in\left[0, T_{\iota}\right]
\end{aligned}
$$

if $\mathbb{P}_{z}\left(\int_{0}^{T}\left(\left|f\left(\mathbf{X}_{t}, Y_{t}^{z}, 0\right)\right|+\left\|g\left(\mathbf{X}_{t}, Y_{t}^{z}, 0\right)\right\|^{2}\right) d t<\infty\right)=1$ and (6.4) is satisfied $\mathbb{P}_{z}$-a.s. 
In much the same way as in the proof of Lemma 3.2 one can show that if (D1)-(D5) are satisfied then for q.e. $z \in E_{0, T}$ the data

$$
(\xi, \bar{f}(t, y, e), \bar{g}(t, y, e))=\left(\varphi\left(\mathbf{X}_{T_{\iota}}\right), f\left(\mathbf{X}_{t}, y, e\right), g\left(\mathbf{X}_{t}, y, e\right)\right), \quad t \in[0, T], y \in \mathbb{R}, e \in \mathbb{R}^{d}
$$

satisfy assumptions (B1)-(B4) under the measure $\mathbb{P}_{z}$. Therefore by Corollary 5.3 and Theorem 5.4, for q.e. $z \in E_{0, T}$ there exists a unique solution $\left(Y^{z}, Z^{z}\right)$ of (6.4).

Theorem 6.2. Assume that (D1)-(D5) are satisfied. Then there exists a unique solution $u$ of SPDE (6.1). Moreover, for q.e. $z \in E_{0, T}$,

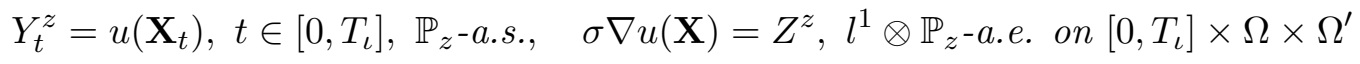

where $\left(Y^{z}, Z^{z}\right)$ is a solution of (6.4).

Proof. The proof of uniqueness is similar to the proof of Proposition 3.9, with obvious modifications in Remark 3.8. Therefore we only show the existence of a solution. Let $\left(Y^{0}, Z^{0}\right)=(0,0)$ and for $n \geq 0$ let $\left(Y^{n+1}, Z^{n+1}\right)$ be a solution of the BDSDE

$$
\begin{aligned}
Y_{t}^{n+1}= & \varphi\left(\mathbf{X}_{T_{\iota}}\right)+\int_{t}^{T_{\iota}} f\left(\mathbf{X}_{r}, Y^{n+1}, Z_{r}^{n}\right) d r+\int_{t}^{T_{\iota}} g\left(\mathbf{X}_{r}, Y_{r}^{n+1}, Z_{r}^{n}\right) d^{\dagger} \beta_{r} \\
& -\int_{t}^{T_{\iota}} Z_{r}^{n+1} d W_{r}, \quad t \in\left[0, T_{\iota}\right], \quad \mathbb{P}_{z^{-} \text {-a.s. }}
\end{aligned}
$$

By Proposition 6.1,

$$
Y_{t}^{n}=u^{n}\left(\mathbf{X}_{t}\right), \quad t \in\left[0, T_{\iota}\right], \quad \mathbb{P}_{z^{-} \text {-a.s. }}
$$

and

$$
\sigma \nabla u^{n}(\mathbf{X})=Z^{n}, \quad l^{1} \otimes \mathbb{P}_{z^{-}} \text {a.e. on }\left[0, T_{\iota}\right] \times \Omega \times \Omega^{\prime}
$$

for q.e. $z \in E_{0, T}$, where $u^{0}=0$ and $u^{n}, n \geq 1$, is a solution of the SPDE

$$
\frac{\partial u^{n}}{\partial t}+A_{t} u^{n}=-f\left(t, x, u^{n}, \sigma \nabla u^{n-1}\right)-g\left(t, x, u^{n}, \sigma \nabla u^{n-1}\right) d^{\dagger} \beta_{t}, \quad u^{n}(T)=\varphi .
$$

We know that for q.e. $z \in E_{0, T}$ there exist a unique solution $\left(Y^{z}, Z^{z}\right)$ of $(6.4)$ and from the proof of Theorem 5.4 it follows that

$$
\lim _{n \rightarrow \infty}\left\|\left(Y^{n}, Z^{n}\right)-\left(Y^{z}, Z^{z}\right)\right\|_{\beta, z}=0,
$$

where the norm $\|\cdot\|_{\beta, z}$ is defined by (5.1) with $\mathbb{E}$ replaced by $\mathbb{E}_{z}$. Applying the Burkholder-Davis-Gundy inequality and using standard argument we get

$$
\lim _{n \rightarrow \infty} \mathbb{E}_{z}\left(\sup _{0 \leq t \leq T_{\iota}}\left|Y_{t}^{n}-Y_{t}^{z}\right|^{2}+\int_{0}^{T_{\iota}}\left|Z_{t}^{n}-Z_{t}^{z}\right|^{2} d t\right)=0
$$

for q.e. $z \in E_{0, T}$. Let us put $u(z)=\lim _{n \rightarrow \infty} u_{n}(z)$ for those $z \in E_{0, T}$ for which it exists in probability $\mathbb{P}_{z}$, and $u(z)=0$ otherwise. Then from (6.5) and (6.8) we conclude that for q.e. $z \in E_{0, T}$,

$$
Y_{t}^{z}=u\left(\mathbf{X}_{t}\right), \quad t \in\left[0, T_{\iota}\right], \quad \mathbb{P}_{z^{-}} \text {a.s. }
$$


By (6.6), for any $\nu \in \hat{S}_{00}$ (for the definition of $\hat{S}_{00}$ see Remark 3.1) we have

$$
E \int_{E_{0, T}}\left(\left|\sigma \nabla\left(u_{n}-u_{m}\right)(z)\right|^{2} \wedge 1\right) \hat{R}^{0, T} \nu(z) d z=\mathbb{E}_{\nu} \int_{0}^{T_{\iota}}\left(\left|Z_{t}^{n}-Z_{t}^{m}\right|^{2} \wedge 1\right) d t,
$$

which by (6.8) converges to zero as $n, m \rightarrow \infty$. Therefore using [28, Theorem 2.5] (see also the remark at the end of Section 4 in [18]) and applying standard argument (see the proof of [8, Theorem 5.2.1]) shows that there is a measurable $v: \Omega \times E_{0, T} \rightarrow \mathbb{R}$ and a subsequence, still denoted by $n$, such that for q.e. $z \in E_{0, T}, \sigma \nabla u_{n}(z) \rightarrow v(z)$ in probability $P$. From this and (6.8) it follows that

$$
v(\mathbf{X})=Z^{z}, \quad l^{1} \otimes \mathbb{P}_{z} \text {-a.e. on }\left[0, T_{\iota}\right] \times \Omega \times \Omega^{\prime}
$$

for q.e. $z \in E_{0, T}$. Hence the pair $(u(\mathbf{X}), v(\mathbf{X}))$ is a solution of (6.4) for q.e $z \in E_{0, T}$. Consequently, (6.3) with $\sigma \nabla u$ replaced by $v$ is satisfied for q.e. $z \in E_{0, T}$. Applying now Proposition 6.1 shows that $u \in M^{2}\left(0, T ; H_{0}^{1}(D)\right)$ and for q.e. $z \in E_{0, T}$,

$$
0=\mathbb{E}_{z}\left\langle\int_{0}^{.}(\sigma \nabla u-v)\left(\mathbf{X}_{t}\right) d W_{t}\right\rangle_{T_{\iota}}=\mathbb{E}_{z} \int_{0}^{T_{\iota}}\left|(\sigma \nabla u-v)\left(\mathbf{X}_{t}\right)\right|^{2} d t,
$$

which completes the proof of the theorem.

\section{Probabilistic and mild solutions of SPDEs}

In this section we adopt the following notation.

- $U$ is a separable real Hilbert space, $\left\{e_{k}\right\}_{k \geq 1}$ is an orthonormal basis of $U, Q$ is a symmetric nonnegative trace class operator on $U$ such that $Q e_{k}=\lambda_{k} e_{k}, k \geq 1$. We will assume that $U \subset H=L^{2}(E ; m) . \quad U_{0}=Q^{1 / 2}(U) \subset U$ is the separable Hilbert space with the inner product $\langle u, v\rangle_{U_{0}}=\left\langle Q^{-1 / 2} u, Q^{-1 / 2} v\right\rangle_{U}$ (Note that $\left\{f_{k}=\sqrt{\lambda_{k}} e_{k}\right\}$ is an orthonormal basis of $\left.U_{0}\right)$.

- $L\left(U_{0}, \mathbb{R}\right)$ is the Banach space of all bounded operators from $U_{0}$ into $\mathbb{R}$ endowed with the supremum norm and $L_{2}\left(U_{0}, \mathbb{R}\right), L_{2}\left(U_{0}, H\right)$ are the spaces of HilbertSchmidt operators from $U_{0}$ into $\mathbb{R}$ and $H$, respectively.

- $B$ is a $Q$-Wiener process defined on some complete probability space $(\Omega, \mathcal{F}, P)$ with values in $U$.

It is known (see, e.g., [7, Chapter 4]) that $B$ has the expansion

$$
B_{t}=\sum_{k=1}^{\infty} \sqrt{\lambda_{k}} \beta_{t}^{k} e_{k}=\sum_{k=1}^{\infty} \beta_{t}^{k} f_{k}, \quad t \geq 0
$$

where $\beta_{t}^{k}=\lambda_{k}^{-1 / 2}\left\langle B_{t}, e_{k}\right\rangle_{U}$ are real valued mutually independent standard Wiener processes on $(\Omega, \mathcal{F}, P)$ (the series above converges in $L^{2}(\Omega, \mathcal{F}, P ; U)$ and $P$-a.s. in $C([0, T] ; U))$.

Suppose we are given $\tilde{g}: \Omega \times E_{T} \times \mathbb{R} \rightarrow \mathbb{R}$ and $A_{t}, \varphi, f$ as in Section 3 . In this section we consider SPDE of the form

$$
d u(t)=-\left(A_{t} u+f(t, x, u)\right) d t-\tilde{g}(t, x, u) d^{\dagger} B_{t}, \quad u(T)=\varphi .
$$

In what follows

$$
g=\left(g_{1}, g_{2}, \ldots\right), \quad g_{k}(t, x, y)=\tilde{g}(t, x, y) \cdot f_{k}(x), \quad(t, x) \in E_{T}, y \in \mathbb{R} .
$$




\subsection{Probabilistic solutions of (7.2)}

We assume that $\varphi, f, g$ satisfy (H1)-(H5). By $u$ we denote the unique solution of SPDE (3.4) of Theorem 3.7.

Let $t \mapsto G\left(\mathbf{X}_{t}, u\left(\mathbf{X}_{t}\right)\right)$ be the process with values in $L\left(U_{0}, \mathbb{R}\right)$ defined as

$$
G\left(\mathbf{X}_{t}, u\left(\mathbf{X}_{t}\right)\right) \psi=\tilde{g}\left(\mathbf{X}_{t}, u\left(\mathbf{X}_{t}\right)\right) \cdot \psi\left(\mathbf{X}_{t}\right), \quad t \geq 0
$$

for $\psi \in U_{0}$. Since $g$ satisfies (H3b), (H4), (H5), and $u(\mathbf{X})$ is of class $M$, it follows from Remark 3.1 that the process $t \mapsto g_{k}\left(\mathbf{X}_{t}, u\left(\mathbf{X}_{t}\right)\right)$ is of class $M$. Since the family $\left\{f_{k} \otimes 1, k \geq 1\right\}$, where $\left(f_{k} \otimes 1\right)(\psi)=\left\langle\psi, f_{k}\right\rangle_{U_{0}}, \psi \in U_{0}$, is linearly dense in $L_{2}\left(U_{0}, \mathbb{R}\right)$ and

$$
\begin{aligned}
\left\langle G\left(t, \mathbf{X}_{t}, u\left(\mathbf{X}_{t}\right)\right), f_{k} \otimes 1\right\rangle_{L^{2}\left(U_{0}, \mathbb{R}\right)} & =\sum_{i=1}^{\infty} G\left(t, \mathbf{X}_{t}, u\left(\mathbf{X}_{t}\right)\right) f_{i} \cdot f_{k} \otimes 1\left(f_{i}\right) \\
& =\tilde{g}\left(t, \mathbf{X}_{t}, u\left(\mathbf{X}_{t}\right)\right) \cdot f_{k}\left(\mathbf{X}_{t}\right)=g_{k}\left(t, \mathbf{X}_{t}, u\left(\mathbf{X}_{t}\right)\right),
\end{aligned}
$$

it follows from [7, Lemma 4.8] that $t \mapsto G\left(\mathbf{X}_{t}, u\left(\mathbf{X}_{t}\right)\right)$ is of class $M$. In particular, for every $\omega^{\prime}$ the process $t \mapsto G\left(\mathbf{X}_{t}, u\left(\mathbf{X}_{t}\right)\right)$ is $\left(\mathcal{F}_{t, T_{\iota}}^{\beta^{\iota}}\right)$-adapted. Moreover,

$$
\mathbb{E}_{z} \int_{0}^{T_{\iota}}\left|G\left(\mathbf{X}_{t}, u\left(\mathbf{X}_{t}\right)\right)\right|_{L_{2}\left(U_{0}, \mathbb{R}\right)}^{2} d t=\mathbb{E}_{z} \int_{0}^{T_{\iota}}\left\|g\left(\mathbf{X}_{t}, u\left(\mathbf{X}_{r}\right)\right)\right\|^{2} d t<\infty
$$

so for every $\omega^{\prime}, t \mapsto G\left(\mathbf{X}_{t}, u\left(\mathbf{X}_{t}\right)\right)$ is an $\left(\mathcal{F}_{t, T_{\iota}}^{\beta^{\iota}}\right)$-adapted $L_{2}\left(U_{0} ; \mathbb{R}\right)$-valued process. One can also check (see [5, Proposition 3.4]) that if we set

$$
B_{t}^{\iota}=\sum_{k=1}^{\infty} \beta_{t+\iota(0)}^{k} f_{k}=B_{t+\iota(0)}, \quad t \in[0, T-\iota(0)]
$$

then

$$
\int_{0}^{t} G\left(\mathbf{X}_{r}, u\left(\mathbf{X}_{r}\right)\right) d^{\dagger} B_{r}^{\iota}=\int_{0}^{t} g\left(\mathbf{X}_{r}, u\left(\mathbf{X}_{r}\right)\right) d^{\dagger} \beta_{r}^{\iota}
$$

for every $t \in[0, T]$. Therefore $u$ of Theorem 3.7 is a probabilistic solution of (7.2) in the sense that for q.e. $z \in E_{0, T}$,

$$
u(z)=E_{z}\left(\varphi\left(\mathbf{X}_{T_{\iota}}\right)+\int_{0}^{T_{\iota}} f\left(\mathbf{X}_{t}, u\left(\mathbf{X}_{t}\right)\right) d t+\int_{0}^{T_{\iota}} G\left(\mathbf{X}_{t}, u\left(\mathbf{X}_{t}\right)\right) d^{\dagger} B_{t}^{\iota}\right), \quad P \text {-a.s. }
$$

By (3.1), this can be written in the form

$$
\begin{aligned}
u(s, x)= & E_{s, x}\left(\varphi\left(X_{T}\right)+\int_{0}^{T-s} f\left(s+t, X_{s+t}, u\left(s+t, X_{s+t}\right)\right) d t\right. \\
& \left.+\int_{0}^{T-s} G\left(s+t, X_{s+t}, u\left(s+t, X_{s+t}\right)\right) d^{\dagger} B_{t}^{s}\right), \quad P \text {-a.s. }
\end{aligned}
$$

Remark 7.1. By $[19,(6.2 .24)]$ and $[18,(4.4)]$, if $m(B)>0$ for some Borel set $B \subset E$ then $\operatorname{cap}(\{s\} \times B)>0$ for every $s \in \mathbb{R}$. From this and the fact that (7.7) holds for q.e. $(s, x) \in E_{0, T}$ it follows that for every $s \in(0, T]$ equality (7.7) holds true for $m$-a.e. $x \in E$. 


\subsection{Mild solutions of (7.2)}

In this subsection we assume additionally that $B^{(t)}=B^{(0)}, t \in[0, T]$, and that $f$ is Lipschitz continuous with respect to $y$, i.e. we assume (H1), (H2), (H3)(a), (H4), (H5) and the following condition: there exists $L>0$ such that for every $z \in E_{0, T}$,

$$
\left|f(z, y)-f\left(z, y^{\prime}\right)\right| \leq L\left|y-y^{\prime}\right| \quad \text { for } y, y^{\prime} \in \mathbb{R} .
$$

Let $A$ denote the operator corresponding to the form $B^{(0)},\left\{P_{t}, t>0\right\}$ denote the semigroup of linear operators on $H$ associated with $B^{(0)}$, and let

$$
F: \Omega \times[0, T] \times H \rightarrow H, \quad G: \Omega \times[0, T] \times H \rightarrow L_{2}\left(U_{0}, H\right)
$$

be operators defined as

$$
F(\omega, t, v)(x)=f(\omega, t, x, v(x)), \quad(G(\omega, t, v) \psi)(x)=\tilde{g}(\omega, t, x, v(x)) \cdot \psi(x)
$$

for $t \in[0, T], v \in H, \psi \in U_{0}, x \in E$. We shall show that $u$ is a mild solution of equation (7.2) interpreted as abstract evolution equation of the form

$$
u(s)=\varphi+\int_{s}^{T}(A u(t)+F(t, u(t))) d t+\int_{s}^{T} G(t, u(t)) d^{\dagger} B_{t}, \quad u(T)=\varphi,
$$

on the Hilbert space $H$, i.e. if we set $u(s)(x)=u(s, x)$ for $(s, x) \in E_{0, T}$ then

$$
u(s)=P_{T-s} \varphi+\int_{s}^{T} P_{t-s} F(t, u(t)) d t+\int_{s}^{T} P_{t-s} G(t, u(t)) d^{\dagger} B_{t} .
$$

Here the integral involving $F$ is Bochner's integral. To see this, let us first note that from (7.8), (H3b) it follows that $F(t, \cdot), G(t, \cdot)$ are Lipschitz-continuous. Using this and [7, Lemma 4.8] one can show that $F$ (resp. $G$ ) is an $\left(\mathcal{F}_{t, T}^{\beta}\right)$-progressively measurable mapping from $\Omega \times[0, T] \times H$ into $(H, \mathcal{B}(H))$ (resp. into $\left(L^{2}\left(U_{0}, H\right), \mathcal{B}\left(L^{2}\left(U_{0}, H\right)\right)\right.$ ). Since $\left\{P_{t}\right\}$ is a contraction on $H$, it follows from (H1), (H3)(b) and (7.8) that there is $c>0$ such that

$$
\left|P_{t} F(t, v)\right|_{H} \leq c\left(1+|v|_{H}\right), \quad\left|P_{t} G(t, v)\right|_{L_{2}\left(U_{0}, H\right)} \leq c\left(1+|v|_{H}\right), \quad t \in[0, T] .
$$

Since $u \in M^{2}(0, T ; H), E \int_{s}^{T}\left(\left|P_{t} F(t, u(t))\right|_{H}+\left|P_{t} G(t, u(t))\right|_{L_{2}\left(U_{0}, H\right)}^{2}\right) d t<\infty$, so the integrals in (7.10) involving $F$ and $G$ are well defined. By [24, Proposition A.2.2], for $h \in H$ we have

$$
\begin{aligned}
\left\langle\int_{s}^{T} P_{t-s} F(t, u(t)) d t, h\right\rangle_{H} & =\int_{s}^{T}\left\langle P_{t-s} F(t, u(t)), h\right\rangle_{H} d t \\
& =\int_{0}^{T-s}\left\langle E_{s, \cdot} f\left(s+t, X_{s+t}, u\left(s+t, X_{s+t}\right)\right), h\right\rangle_{H} d t \\
& =\left\langle\int_{0}^{T-s} E_{s, \cdot} f\left(s+t, X_{s+t}, u\left(s+t, X_{s+t}\right)\right) d t, h\right\rangle_{H} .
\end{aligned}
$$

By the above and Fubini's theorem,

$$
\left\langle\int_{s}^{T} P_{t-s} F(t, u(t)) d t, h\right\rangle_{H}=\left\langle E_{s, \cdot} \int_{0}^{T-s} f\left(s+t, X_{s+t}, u\left(s+t, X_{s+t}\right) d t, h\right\rangle_{H} .\right.
$$


Similarly, by [24, Lemma 2.4.1] and the fact that the process $X$ it time-homogeneous,

$$
\begin{gathered}
\left\langle\int_{s}^{T} P_{t-s} G(t, u(t)) d^{\dagger} B_{t}, h\right\rangle_{H}=\sum_{k=1}^{\infty} \int_{s}^{T}\left\langle P_{t-s} G(t, u(t))\left(f_{k}\right), h\right\rangle_{H} d^{\dagger} \beta_{t}^{k} \\
=\sum_{k=1}^{\infty} \int_{0}^{T-s}\left\langle E_{s, \cdot} g_{k}\left(s+t, X_{s+t}, u\left(s+t, X_{s+t}\right), h\right\rangle_{H} d^{\dagger} \beta_{t+s}^{k}\right. \\
=\sum_{k=1}^{\infty}\left\langle\int_{0}^{T-s} E_{s, \cdot} g_{k}\left(s+t, X_{s+t}, u\left(s+t, X_{s+t}\right) d^{\dagger} \beta_{t+s}^{k}, h\right\rangle_{H} .\right.
\end{gathered}
$$

By the above and the stochastic Fubini theorem (see [7, Theorem 4.18]),

$$
\begin{aligned}
\left\langle\int_{s}^{T}\right. & \left.P_{t-s} G(t, u(t)) d^{\dagger} B_{t}, h\right\rangle_{H} \\
& =\sum_{k=1}^{\infty}\left\langle E_{s, \cdot} \int_{0}^{T-s} g_{k}\left(s+t, X_{s+t}, u\left(s+t, X_{s+t}\right) d^{\dagger} \beta_{t+s}^{k}, h\right\rangle_{H}\right. \\
& =\left\langle E_{s, \cdot} \sum_{k=1}^{\infty} \int_{0}^{T-s} g_{k}\left(s+t, X_{s+t}, u\left(s+t, X_{s+t}\right)\right) d^{\dagger} \beta_{s+t}^{k}, h\right\rangle_{H} \\
& =\left\langle E_{s, \cdot} \int_{0}^{T-s} G\left(s+t, X_{s+t}, u\left(s+t, X_{s+t}\right)\right) d^{\dagger} B_{t}^{s}, h\right\rangle_{H} .
\end{aligned}
$$

From Remark 7.1 and (7.11), (7.12) it follows that $\langle u(s), h\rangle_{H}=\langle v(s), h\rangle_{H}$ for $h \in H$, where $v(s)$ is defined by the right-hand side of (7.10). This shows that $u$ satisfies (7.10). Thus we have proved he following proposition.

Proposition 7.2. Assume that $B^{(t)}=B^{(0)}$ for all $t \in[0, T]$, and $(\mathrm{H} 1)-(\mathrm{H} 5),(7.8)$ are satisfied. Let $u$ be the unique solution of SPDE (3.4). Then (7.10) holds true for every $s \in(0, T]$.

Remark 7.3. Let

$$
\hat{B}_{t}=B_{T-t}-B_{T}, \quad t \in[0, T]
$$

On can check that

$$
\int_{T-s}^{T} P_{t-(T-s)} G(t, u(t)) d^{\dagger} B_{t}=-\int_{0}^{s} P_{s-t} G(T-t, u(T-t)) d \hat{B}_{t}, \quad s \in[0, T] .
$$

Therefore from (7.10) it follows that $\bar{u}$ defined as $\bar{u}(t)=u(T-t), t \in[0, T]$, is a mild solution of the problem

$$
d \bar{u}(t)=(A \bar{u}+f(T-t, x, \bar{u})) d t-\tilde{g}(T-t, x, \bar{u}) d \hat{B}_{t}, \quad \bar{u}(0)=\varphi .
$$

Remark 7.4. Note that in case $A_{t}$ is defined by (1.2) one can generalize (7.6) to equations of the form (7.2) with $f, \tilde{g}$ also depending on the gradient of a solution (see (6.1)) and satisfying (D1)-(D5). If, in addition, $A_{t}=A_{0}, t \in[0, T]$, and we replace (D3)(a) by Lipschitz continuity in $y$, then in much the same way as in the proof of (7.10) one can prove that $u$ of Theorem 6.2 is a mild solution of (7.9) with the mappings $F: \Omega \times[0, T] \times H_{0}^{1}(D) \rightarrow H, G: \Omega \times[0, T] \times H_{0}^{1}(D) \rightarrow L_{2}\left(U_{0}, H\right)$ defined as

$$
\begin{gathered}
F(\omega, t, v)(x)=f(\omega, t, x, v(x), \sigma \nabla v(x)), \\
(G(\omega, t, v) \psi)(x)=\tilde{g}(\omega, t, x, v(x), \sigma \nabla v(x)) \cdot \psi(x)
\end{gathered}
$$

for $t \in[0, T], v \in H_{0}^{1}(D), \psi \in U_{0}, x \in D$. 


\subsection{Examples}

Assume that $e_{k} \in L^{\infty}(E ; m)$ and $e_{k}$ are bounded uniformly in $k$, or, more generally,

$$
\sup _{x \in E} \sum_{k=1}^{\infty} \lambda_{k}\left|e_{k}(x)\right|^{2}<\infty .
$$

Below we show that under (7.13) the results of Sections 7.1 and 7.2 apply to equation (7.2) with $\tilde{g}$ Lipschitz continuous such that $\tilde{g}(\cdot, 0)$ is bounded or square integrable. For simplicity we assume that $\tilde{g}$ does not depend on $\omega$.

Example 7.5. Assume that

$$
\tilde{g}(\cdot, 0) \in L^{2}\left(E_{0, T} ; m_{T}\right) \cup L^{\infty}\left(E_{0, T} ; m_{T}\right)
$$

and $\tilde{g}$ is Lipschitz continuous in $y$ with some constant $L^{\prime}$, i.e.

$$
\left|\tilde{g}\left(t, x, y_{1}\right)-\tilde{g}\left(t, x, y_{2}\right)\right| \leq L^{\prime}\left|y_{1}-y_{2}\right|
$$

for all $(t, x) \in E_{0, T}$ and $y_{1}, y_{2} \in \mathbb{R}$. Then $g$ defined by (7.3) satisfies (H1) and (H3). Indeed, we have

$$
\left\|g_{k}(\cdot, 0)\right\|_{L^{2}\left(E_{0, T} ; m_{T}\right)}^{2}=\lambda_{k}\left\|\tilde{g}(\cdot, 0) e_{k}(\cdot)\right\|_{L^{2}\left(E_{0, T} ; m_{T}\right)}^{2}
$$

and

$$
\left|g_{k}(t, x, y)-g_{k}\left(t, x, y^{\prime}\right)\right| \leq L^{\prime} \sqrt{\lambda_{k}}\left|e_{k}(x)\right| .
$$

By the last inequality, (H3) is satisfied with $L_{k}(x)=L^{\prime} \sqrt{\lambda_{k}}\left|e_{k}(x)\right|$. In case $\tilde{g}(\cdot, 0) \in$ $L^{2}\left(E_{0, T} ; m_{T}\right)$ assumption (7.13) immediately forces $g$ to satisfy (H1). If $\tilde{g}(\cdot, 0) \in$ $L^{\infty}\left(E_{0, T} ; m_{T}\right)$ then $\left\|\tilde{g}(\cdot, 0) e_{k}(\cdot)\right\|_{L^{2}\left(E_{0, T} ; m_{T}\right)}^{2} \leq T^{2}\|\tilde{g}(\cdot, 0)\|_{\infty}\left\|e_{k}\right\|_{H}$, so $g$ satisfies (H3), because $\operatorname{Tr} Q<\infty$ and we assume that $U \subset H$.

Example 7.6. Let $D$ be a nonempty bounded open subset of $\mathbb{R}^{d}$ and let $\tilde{g}: D_{0, T} \times$ $\mathbb{R} \times \mathbb{R}^{d} \rightarrow \mathbb{R}$ be a measurable function. If $\tilde{g}(\cdot, 0,0) \in L^{2}\left(D_{0, T} ; m_{T}\right) \cup L^{\infty}\left(D_{0, T} ; m_{T}\right)$ and

$$
\left|\tilde{g}\left(t, x, y_{1}, e_{1}\right)-\tilde{g}\left(t, x, y_{2}, e_{2}\right)\right| \leq L^{\prime}\left(\left|y_{1}-y_{2}\right|+\left|e_{1}-e_{2}\right|\right)
$$

for every $(t, x) \in E_{0, T}$ and $y_{1}, y_{2} \in \mathbb{R}, e_{1}, e_{2} \in \mathbb{R}^{d}$ then $g=\left(g_{1}, g_{2}, \ldots\right)$ defined by $g_{k}(t, x, y, e)=\tilde{g}(t, x, y, e) \cdot f_{k}(x)$ satisfies (D1) and (D3) with $L_{k}(x)=M_{k}(x)=$ $L^{\prime} \sqrt{\lambda_{k}}\left|e_{k}(x)\right|, x \in D$.

\section{Acknowledgements}

This research was supported by NCN Grant No. 2012/07/B/ST1/03508.

\section{References}

[1] V. Bally, I. Gyöngy and E. Pardoux, White noise driven parabolic SPDEs with measurable drift, J. Funct. Anal. 120 (1994), 484-510.

[2] V. Bally and A. Matoussi, Weak solutions for SPDEs and backward doubly stochastic differential equations, J. Theoret. Probab. 14 (2001), 125-164. 
[3] B. Boufoussi, J. Van Casteren and M. Mrhardy, Generalized backward doubly stochastic differential equations and SPDEs with nonlinear Neumann boundary conditions, Bernoulli 13 (2007), 423-446.

[4] Ph. Briand, B. Delyon, Y. Hu, E. Pardoux and L. Stoica, $L^{p}$ solutions of Backward Stochastic Differential Equations, Stochastic Process. Appl. 108 (2003), 109-129.

[5] R.C. Dalang and L. Quer-Sardanyons, Stochastic integrals for spde's: a comparison, Expo. Math. 29 (2011), 67-109.

[6] L. Denis and L. Stoica, A general analytical result for non-linear SPDE's and applications, Electron. J. Probab. 9 (2004) No. 23, 674-709.

[7] G. Da Prato and J. Zabczyk, Stochastic Equations in Infinite Dimensions, Cambridge University Press, Cambridge, 1992.

[8] M. Fukushima, Y. Oshima and M. Takeda, Dirichlet Forms and Symmetric Markov Processes, Walter de Gruyter, Berlin, 1994.

[9] I. Gyöngy and E. Pardoux, On the regularization effect of space-time white noise on quasi-linear parabolic partial differential equations, Probab. Theory Related Fields 97 (1993), 211-229.

[10] O. Kallenberg, Foundations of modern probability. Second edition, SpringerVerlag, New York, 2002.

[11] T. Klimsiak, Reflected BSDEs and the obstacle problem for semilinear PDEs in divergence form, Stochastic Process. Appl. 122 (2012), 134-169

[12] T. Klimsiak, Semi-Dirichlet forms, Feynman-Kac functionals and the Cauchy problem for semilinear parabolic equations, J. Funct. Anal. 268 (2015), 12051240 .

[13] T. Klimsiak and A. Rozkosz, Dirichlet forms and semilinear elliptic equations with measure data, J. Funct. Anal. 265 (2013), 890-925.

[14] T. Klimsiak and A. Rozkosz, Semilinear elliptic equations with measure data and quasi-regular Dirichlet forms, Colloq. Math. (2016), DOI: 10.4064/cm6466-102015 .

[15] N.V. Krylov, An analytic approach to SPDEs. Stochastic partial differential equations: six perspectives, in: Math. Surveys Monogr., 64, Amer. Math. Soc., Providence, RI, 1999, 185-242.

[16] Z. Ma and Z. Röckner, Introduction to the Theory of (Non-Symmetric) Dirichlet Forms, Springer-Verlag, Berlin, 1992.

[17] Y. Oshima, Some Properties of Markov processes associated with time dependent Dirichlet forms, Osaka J. Math. 29 (1992), 103-127.

[18] Y. Oshima, Time-dependent Dirichlet forms and related stochastic calculus, Infin. Dimens. Anal. Quantum Probab. Relat. Top. 7 (2004), 281-316. 
[19] Y. Oshima, Semi-Dirichlet Forms and Markov Processes, Walter de Gruyter, Berlin, 2013.

[20] E. Pardoux and S. Peng, Backward doubly stochastic differential equations and systems of quasilinear SPDEs, Probab. Theory Related Fields 98 (1994), 209227.

[21] S. Peszat and J. Zabczyk, Stochastic evolution equations with a spatially homogeneous Wiener process, Stochastic Process. Appl. 72 (1997), 187-204.

[22] S. Peszat and J. Zabczyk, Nonlinear stochastic wave and heat equations, Probab. Theory Related Fields 116 (2000), 421-443.

[23] M. Pierre, Parabolic Capacity and Sobolev Spaces, J. Math. Anal. 14 (1983), $522-533$.

[24] C. Prévôt and M. Röckner, A Concise Course on Stochastic Partial Differential Equations, Lecture Notes in Math. 1905, Springer, Berlin, 2007.

[25] B.L. Rozovskii, Stochastic evolution systems, Kluwer, Dordrecht, 1990.

[26] M. Sharpe, General Theory of Markov Processes. Academic Press, New York, 1988.

[27] W. Stannat, The theory of generalized Dirichlet forms and its applications in analysis and stochastics, Mem. Amer. Math. Soc. 142 (1999), no. 678.

[28] G. Trutnau, Stochastic Calculus of Generalized Dirichlet Forms and Applications to Stochastic Differential Equations in Infinite Dimensions, Osaka J. Math. 37 (2000), 315-343.

[29] J.B. Walsh, An introduction to stochastic partial differential equations, in: École d'été de probabilités de Saint-Flour, XIV-1984, Lecture Notes in Math. 1180, Springer, Berlin, 1986, 265-439.

[30] Z. Wu and F. Zhang, BDSDEs with locally monotone coefficients and Sobolev solutions for SPDEs, J. Differential Equations 251 (2011), 759-784.

[31] Q. Zhang and H. Zhao, Stationary solutions of SPDEs and infinite horizon BDSDEs, J. Funct. Anal. 252, (2007) 171-219.

Tomasz Klimsiak: Institute of Mathematics, Polish Academy of Sciences, Śniadeckich 8, 00-956 Warszawa, Poland

and

Faculty of Mathematics and Computer Science, Nicolaus Copernicus University, Chopina 12/18, 87-100 Toruń, Poland

E-mail: tomas@mat.umk.pl

Andrzej Rozkosz: Faculty of Mathematics and Computer Science, Nicolaus Copernicus University, Chopina 12/18, 87-100 Toruń, Poland

E-mail: rozkosz@mat.umk.pl 